\title{
Rough Solutions of the Einstein Constraints on Closed Manifolds without Near-CMC Conditions
}

\author{
Michael Holst $^{\star}$, Gabriel Nagy ${ }^{\star \star}$, Gantumur Tsogtgerel ${ }^{\star \star}$ \\ Department of Mathematics, University of California San Diego, La Jolla, CA 92093, USA. \\ E-mail: mholst@math.ucsd.edu; gnagy@math.ucsd.edu; gantumur@math.ucsd.edu
}

Received: 12 April 2008 / Accepted: 15 October 2008

Published online: 26 February 2009 - (C) The Author(s) 2009. This article is published with open access at Springerlink.com

\begin{abstract}
We consider the conformal decomposition of Einstein's constraint equations introduced by Lichnerowicz and York, on a closed manifold. We establish existence of non-CMC weak solutions using a combination of a priori estimates for the individual Hamiltonian and momentum constraints, barrier constructions and fixed-point techniques for the Hamiltonian constraint, Riesz-Schauder theory for the momentum constraint, together with a topological fixed-point argument for the coupled system. Although we present general existence results for non-CMC weak solutions when the rescaled background metric is in any of the three Yamabe classes, an important new feature of the results we present for the positive Yamabe class is the absence of the near-CMC assumption, if the freely specifiable part of the data given by the traceless-transverse part of the rescaled extrinsic curvature and the matter fields are sufficiently small, and if the energy density of matter is not identically zero. In this case, the mean extrinsic curvature can be taken to be an arbitrary smooth function without restrictions on the size of its spatial derivatives, so that it can be arbitrarily far from constant, giving what is apparently the first existence results for non-CMC solutions without the near-CMC assumption. Using a coupled topological fixed-point argument that avoids near-CMC conditions, we establish existence of coupled non-CMC weak solutions with (positive) conformal factor $\phi \in W^{s, p}$, where $p \in(1, \infty)$ and $s(p) \in(1+3 / p, \infty)$. In the CMC case, the regularity can be reduced to $p \in(1, \infty)$ and $s(p) \in(3 / p, \infty) \cap[1, \infty)$. In the case of $s=2$, we reproduce the CMC existence results of Choquet-Bruhat [10], and in the case $p=2$, we reproduce the CMC existence results of Maxwell [33], but with a proof that goes through the same analysis framework that we use to obtain the non-CMC results. The non-CMC results on closed manifolds here extend the 1996 non-CMC result of Isenberg and Moncrief in three ways: (1) the near-CMC assumption is removed in the case of the positive Yamabe class; (2) regularity is extended down to the maximum
\end{abstract}

\footnotetext{
* Supported in part by NSF Awards 0715146, 0411723, and 0511766, and DOE Awards DE-FG02-05ER25707 and DE-FG02-04ER25620.

$\star \star$ Supported in part by NSF Awards 0715146 and 0411723.
} 
allowed by the background metric and the matter; and (3) the result holds for all three Yamabe classes. This last extension was also accomplished recently by Allen, Clausen and Isenberg, although their result is restricted to the near-CMC case and to smoother background metrics and data.

\section{Contents}

1. Introduction . . . . . . . . . . . . . . . . . . . . 548

2. Preliminary Material . . . . . . . . . . . . . . . . . . 552

3. Overview of the Main Results . . . . . . . . . . . . . . . . . . . . 559

4. Weak Solution Results for the Individual Constraints . . . . . . . . . . . . . 568

5. Barriers for the Hamiltonian Constraint . . . . . . . . . . . . . . . . . . . . 574

6. Proof of the Main Results . . . . . . . . . . . . . . . . . . . . . 585

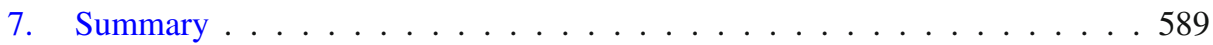

A. Some Key Technical Tools and Some Supporting Results . . . . . . . . . . 591

\section{Introduction}

In this article, we give an analysis of the coupled Hamiltonian and momentum constraints in the Einstein equations on a 3-dimensional closed manifold. We consider the equations with matter sources satisfying an energy condition implied by the dominant energy condition in the 4-dimensional spacetime; the unknowns are a Riemannian three-metric and a two-index symmetric tensor. The equations form an under-determined system; therefore, we focus entirely on a standard reformulation used in both mathematical and numerical general relativity, called the conformal method, introduced by Lichnerowicz and York $[32,49,50]$. The conformal method assumes that the unknown metric is known up to a scalar field called a conformal factor, and also assumes that the trace and a term proportional to the trace-free divergence-free part of the two-index symmetric tensor is known, leaving as unknown a term proportional to the traceless symmetrized derivative of a vector. Therefore, the new unknowns are a scalar and a vector field, transforming the original under-determined system for a metric and a symmetric tensor into a (potentially) well-posed elliptic system for a scalar and a vector field. See [5] for a recent review article.

The question of existence of solutions to the Lichnerowicz-York conformally rescaled Einstein's constraint equations, for an arbitrarily prescribed mean extrinsic curvature, has remained an open problem for more than thirty years. The rescaled equations, which are a coupled nonlinear elliptic system consisting of the scalar Hamiltonian constraint coupled to the vector momentum constraint, have been studied almost exclusively in the setting of constant mean extrinsic curvature, known as the CMC case. In the CMC case the equations decouple, and it has long been known how to establish existence of solutions. The case of CMC data on closed (compact without boundary) manifolds was completely resolved by several authors over the last twenty years, with the last remaining sub-cases resolved and all the CMC sub-cases on closed manifolds summarized by Isenberg in [25]. Over the last ten years, other CMC cases on different types of manifolds containing various kinds of matter fields were studied and partially or completely resolved; see the survey [5]. We take a moment to point out just some of the quite substantial number of works in this area, including: the original work on the Lichnerowicz equation [32]; the development of the conformal method [49-52]; the initial solution theory for the Hamiltonian constraint [39-41]; the thin sandwich alternative 
to the conformal method [4,37]; the complete classification of CMC initial data [25] and the few known non-CMC results [11,26,28]; various technical results on transversetraceless tensors and the conformal Killing operator $[6,8]$; the more recent development of the conformal thin sandwich formulation [53]; initial data for black holes [7,9]; initial data for Kerr-like black holes [13,14]; initial data with trapped surface boundaries $[15,34]$; rough solution theory for CMC initial data $[10,33,35]$; and the gluing approach to generating initial data [12]. A survey of many of these results appears in [5].

On the other hand, the question of existence of solutions to the Einstein constraint equations for non-constant mean extrinsic curvature (the "non-CMC case") has remained largely unanswered, with progress made only in the case that the mean extrinsic curvature is nearly constant (the "near-CMC case"), in the sense that the size of its spatial derivatives is sufficiently small. The near-CMC condition leaves the constraint equations coupled, but ensures the coupling is weak. In [26], Isenberg and Moncrief established the first existence (and uniqueness) result in the near-CMC case, for background metric having negative Ricci scalar. Their result was based on a fixed-point argument, together with the use of iteration barriers (sub- and super-solutions) which were shown to be bounded above and below by fixed positive constants, independent of the iteration. We note that both the fixed-point argument and the global barrier construction in [26] rely critically on the near-CMC assumption. All subsequent non-CMC existence results are based on the framework in [26] and are thus limited to the near-CMC case (see the survey [5], the non-existence results in [27], and also the newer existence results in [1] for non-negative Yamabe classes).

This article presents (together with the brief overview in [22]) the first non-CMC existence results for the Einstein constraints that do not require the near-CMC assumption. Two recent advances make this possible: A new topological fixed-point argument (established here and in [21]) and a new global super-solution construction for the Hamiltonian constraint (established here and in [22]) that are both free of near-CMC conditions. These two results allow us to establish existence of non-CMC solutions for conformal background metrics in the positive Yamabe class, with the freely specifiable part of the data given by the traceless-transverse part of the rescaled extrinsic curvature and the matter fields sufficiently small, and with the matter energy density not identically zero. Our results here and in $[21,22]$ can be viewed as reducing the remaining open questions of existence of non-CMC (weak and strong) solutions without near-CMC conditions to two more basic and clearly stated open problems: (1) Existence of near-CMC-free global super-solutions for the Hamiltonian constraint equation when the background metric is in the non-positive Yamabe classes and for large data; and (2) existence of near-CMCfree global $s u b$-solutions for the Hamiltonian constraint equation when the background metric is in the positive Yamabe class in vacuum (without matter). We will make some further comments about this later in the paper.

Our results in this article, which can be viewed as pushing forward the rough solutions program that was initiated by Maxwell in [33,35] (see also [10]), further extend the known solution theory for the Einstein constraint equations on closed manifolds in several directions:

(i) Far-from-CMC Weak Solutions: We establish the first existence results (Theorem 1) for the coupled Einstein constraints in the non-CMC setting without the near-CMC condition. In particular, if the rescaled background metric is in the positive Yamabe class, if the freely specifiable part of the data given by the traceless-transverse part of the rescaled extrinsic curvature and the matter fields are sufficiently small, and if the energy density of matter is not identically zero, then 
we show existence of non-CMC solutions with mean extrinsic curvature arbitrarily far from constant. Two advances in the analysis of the Einstein constraint equations make this result possible: A topological fixed-point argument (Theorems 4 and 5) based on compactness arguments rather than $k$-contractions that is free of near-CMC conditions, and constructions of global barriers for the Hamiltonian constraint that are similarly free of the near-CMC condition (Lemmas 7, 8, 9, 13, and 14).

(ii) Near-CMC Weak Solutions: We establish existence results (Theorem 2) for non$\mathrm{CMC}$ solutions to the coupled constraints under the near-CMC condition in the setting of weaker (rougher) solutions spaces and for more general physical scenarios than appeared previously in [26,1]. In particular, we establish existence of weak solutions to the coupled Hamiltonian and momentum constraints on closed manifolds for all three Yamabe classes, with (positive) conformal factor in $\phi \in W^{s, p}$, where $p \in(1, \infty)$ and $s(p) \in(1+3 / p, \infty)$. These results are based on combining barriers, a priori estimates, and other results for the individual constraints together with a new type of topological fixed-point argument (Theorems 4 and 5), and are established in the presence of a weak background metric and data meeting very low regularity requirements.

(iii) CMC Weak Solutions: In the CMC case, we establish existence (Theorem 3) of weak solutions to the un-coupled Hamiltonian and momentum constraints on closed manifolds for all three Yamabe classes, with (positive) conformal factor $\phi \in W^{s, p}$, where $p \in(1, \infty)$ and $s(p) \in(3 / p, \infty) \cap[1, \infty)$. In the case of $s=2$, we reproduce the CMC existence results of Choquet-Bruhat [10], and in the case $p=2$, we reproduce the CMC existence results of Maxwell [33], but with a different proof; our CMC proof goes through the same analysis framework that we use to obtain the non-CMC results (Theorems 4 and 5). Again, these results are established in the presence of a weak background metric and with data meeting very low regularity requirements.

(iv) Barrier Constructions: We give constructions (Lemmas 9 and 13) of weak global sub- and super-solutions (barriers) for the Hamiltonian constraint equation which are free of the near-CMC condition. The constructions require the assumption that the freely specifiable part of the data given by the traceless-transverse part of the rescaled extrinsic curvature and the matter fields are sufficiently small (required for the super-solution construction in Lemma 9) and if the energy density of matter is not identically zero (required for the sub-solution in construction Lemma 13, although we note this can be relaxed using the technique in [1]). While near-CMC-free sub-solutions are common in the literature, our near-CMC-free super-solution constructions appear to be the first such results of this type.

(v) Supporting Technical Tools: We assemble a number of new supporting technical results in the body of the paper and in several appendices, including: topological fixed-point arguments designed for the Einstein constraints; construction and properties of general Sobolev classes $W^{s, p}$ and elliptic operators on closed manifolds with weak metrics; the development of a very weak solution theory for the momentum constraint; a priori $L^{\infty}$-estimates for weak $W^{1,2}$-solutions to the Hamiltonian constraint; Yamabe classification of non-smooth metrics in general Sobolev classes $W^{s, p}$; and an analysis of the connection between conformal rescaling and the near-CMC condition.

The results in this paper imply that the weakest differentiable solutions of the Einstein constraint equations we have found correspond to CMC and non-CMC hypersurfaces 
with physical spatial metric $h_{a b}$ satisfying

$$
h_{a b} \in W^{s, p}(\mathcal{M}), \quad p \in(1, \infty), \quad s(p) \in\left(1+\frac{3}{p}, \infty\right)
$$

The curvature of such metrics can be computed in a distributional sense, following [17]. In the $\mathrm{CMC}$ case, the regularity can be reduced to

$$
h_{a b} \in W^{s, p}(\mathcal{M}), \quad p \in(1, \infty), \quad s(p) \in\left(\frac{3}{p}, \infty\right) \cap[1, \infty) .
$$

In the case $s=2$, we reproduce the CMC existence results of Choquet-Bruhat [10], and in the case $p=2$, we reproduce the CMC existence results of Maxwell [33], but with a different proof; our CMC proof goes through the same analysis framework that we use to obtain the non-CMC results (Theorems 4 and 5). In this paper we do not include uniqueness statements on CMC solutions, or necessary and sufficient conditions for the existence of CMC solutions; however, we expect that the techniques used in the above mentioned works can be adapted to this setting without difficulty.

There are several related motivations for establishing the extensions outlined above. First, as outlined in [5], new results for the non-CMC case, beyond the case analyzed in $[1,26]$, are of great interest in both mathematical and numerical relativity. Non-CMC results that are free of the near-CMC assumption are of particular interest, since the existence of solutions in this case has been an open question for more than thirty years. Second, there is currently substantial research activity in rough solutions to the Einstein evolution equations, which rest on rough/weak solution results for the initial data [30]. Third, the approximation theory for Petrov-Galerkin-type methods (including finite element, wavelet, spectral, and other methods) for the constraints and similar systems previously developed in [20] establishes convergence of numerical solutions in very general physical situations, but rests on assumptions about the solution theory; the results in the present paper and in [21], help to complete this approximation theory framework. Similarly, very recent results on convergence of adaptive methods for the constraints in $[23,24]$ rest in large part on the collection of results here and in [20,21].

An extended outline of the paper is as follows.

In Sect. 2, we summarize the conformal decomposition of Einstein's constraint equations introduced by Lichnerowicz and York, on a closed manifold. We describe the classical strong formulation of the resulting coupled elliptic system, and then define weak formulations of the constraint equations that will allow us to develop solution theories for the constraints in the spaces with the weakest possible regularity.

After setting up the basic notation, we give an overview of our main results in Sect. 3, summarized in three existence theorems (Theorems 1, 2, and 3) for weak far-from-CMC, near-CMC, and CMC solutions to the coupled constraints, extending the known solution theory in several distinct ways as described above. We outline the two recent advances in the analysis of the Einstein constraint equations that make these results possible. The first advance is an abstract coupled topological fixed-point result (Theorems 4 and 5), the proof of which is based directly on compactness rather than on $k$-contractions. This gives an analysis framework for weak solutions to the constraint equations that is fundamentally free of the near-CMC assumption; the near-CMC assumption then only potentially arises in the construction of global barriers as part of the overall fixed-point argument. A result of this type also makes possible the new non-CMC results for the case of compact manifolds with boundary appearing in [21]. The second new advance is the construction 
of global super-solutions for the Hamiltonian constraint that are also free of the nearCMC condition; we give an overview of the main ideas in the constructions, which are then derived rigorously in Sect. 5 .

In Sect. 4 we then develop the necessary results for the individual constraint equations in order to complete an existence argument for the coupled system based on the abstract fixed-point argument in Theorems 4 and 5. In particular, in Sect. 4.1, we first develop some basic technical results for the momentum constraint operator under weak assumptions on the problem data, including existence of weak solutions to the momentum constraint, given the conformal factor as data. In Sect. 4.2, we assume the existence of barriers (weak sub- and super-solutions) to the Hamiltonian constraint equation forming a nonempty positive bounded interval, and then derive several properties of the Hamiltonian constraint that are needed in the analysis of the coupled system. The results are established under weak assumptions on the problem data, and for any Yamabe class.

Using order relations on appropriate Banach spaces, we then derive several such compatible weak global sub- and super-solutions in Sect. 5, based both on constants and on more complex non-constant constructions. While the sub-solutions are similar to those found previously in the literature, some of the super-solutions are new. In particular, we give two super-solution constructions that do not require the near-CMC condition. The first is constant, and requires that the scalar curvature be strictly globally positive. The second is based on a scaled solution to a Yamabe-type problem, and is valid for any background metric in the positive Yamabe class.

In Sect. 6, we establish the main results by giving the proofs of Theorems 1, 2, and 3. In particular, using the topological fixed-point argument in Theorem 5, we combine the global barrier constructions in Sect. 5 with the individual constraint results in Sect. 4 to establish existence of weak non-CMC solutions. We summarize our results in Sect. 7. For ease of exposition, various supporting technical results are given in several appendices as follows: Appendix Sect. A.1 - topological fixed-point arguments; Appendix Sect. A. 2 - ordered Banach spaces; Appendix Sect. A.3 - monotone increasing maps; Appendix Sect. A.4 - construction of fractional order Sobolev spaces of sections of vector bundles over closed manifolds; Appendix Sect. A.5 - a priori estimates for elliptic operators; Appendix Sect. A.6 - maximum principles on closed manifolds; Appendix Sect. A.7 - Yamabe classification of weak metrics; Appendix Sect. A.8 - conformal covariance of the Hamiltonian constraint; and Appendix Sect. A.9 - conformal rescaling and the near-CMC condition.

\section{Preliminary Material}

2.1. Notation and conventions. Let $\mathcal{M}$ be an $n$-dimensional smooth closed manifold. We denote by $\pi: E \rightarrow \mathcal{M}$ (or simply $E \rightarrow \mathcal{M}$, or just $E$ ) a smooth vector bundle over $\mathcal{M}$, where the manifold $\mathcal{M}$ is called the base space, $E$ is called the total space, and $\pi$ is the bundle projection such that for any $x \in \mathcal{M}, E_{x}=\pi^{-1}(x)$ is the fiber over $x$, which is a vector space of (fiber) dimension $m_{x}$. If all fibers $E_{x}$ have dimension $m_{x}=m$, we say the fiber dimension of $E$ is $m$. The manifold $\mathcal{M}$ itself can be considered as the vector bundle $E=\mathcal{M} \times\{0\}$ with fiber dimension $m=0$. A section of the trivial vector bundle $E=\mathcal{M} \times \mathbb{R}$ with fiber dimension $m=1$ is simply a scalar function on $\mathcal{M}$. Our primary interest is the case where

$$
E=T_{s}^{r} \mathcal{M}=\underbrace{T \mathcal{M} \otimes \cdots \otimes T \mathcal{M}}_{r \text { times }} \otimes \underbrace{T^{*} \mathcal{M} \otimes \ldots \otimes T^{*} \mathcal{M}}_{s \text { times }},
$$


the $(r, s)$-tensor bundle with contravariant order $r$ and covariant order $s$, giving fiber dimension $m=n(r+s)$, where $T \mathcal{M}$ is the tangent bundle, and $T^{*} \mathcal{M}$ is the co-tangent bundle of $\mathcal{M}$. A $C^{k}$ section of $\pi$ (or of $E$ ) is a $C^{k}$ map $\gamma: \mathcal{M} \rightarrow E$ such that for each $x \in \mathcal{M}, \pi(\gamma(x))=x$. These $C^{k}$ sections form real Banach spaces $C^{k}(E)$ which arise naturally in the global linear analysis of partial differential equations on manifolds.

Let $h_{a b} \in C^{\infty}\left(T_{2}^{0} \mathcal{M}\right)$ be a smooth Riemannian metric on $\mathcal{M}$, (where by convention Latin indices denote abstract indices as e.g. in [48]), meaning that it is a symmetric, positive definite, covariant, smooth two-index tensor field on $\mathcal{M}$. The combination $\left(\mathcal{M}, h_{a b}\right)$ is referred to as a (smooth) Riemannian manifold; we will relax the smoothness requirement on $h_{a b}$ below. For each $x \in \mathcal{M}$, the metric $h_{a b}(x)$ defines a positive definite inner product on the tangent space $T_{x} \mathcal{M}$ at $x$. Denote by $h^{a b}$ the inverse of $h_{a b}$, that is, $h_{a c} h^{b c}=\delta_{a}{ }^{b}$, where $\delta_{a}{ }^{b}: T_{x} \mathcal{M} \rightarrow T_{x} \mathcal{M}$ is the identity map. We use the convention that repeated indices, one upper-index and one sub-index, denote contraction. Indices on tensors will be raised and lowered with $h^{a b}$ and $h_{a b}$, respectively. For example, given the tensor $u^{a b}{ }_{c}$ we denote $u_{a b c}=h_{a a_{1}} h_{b b_{1}} u^{a_{1} b_{1}}{ }_{c}$, and $u^{a b c}=h^{c c_{1}} u^{a b}{ }_{c_{1}}$; notice that the order of the indices is important in the case that the tensor $u_{a b c}$ or $u^{a b c}$ is not symmetric. We say that a tensor is of type $m$ iff it can be transformed into a tensor $u_{a_{1} \cdots a_{m}}$ by lowering appropriate indices (its vector bundle then has fiber dimension $m n$ ).

We now give a brief overview of $L^{p}$ and Sobolev spaces of sections of vector bundles over closed manifolds in order to introduce the notation used throughout the paper. An overview of the construction of fractional order Sobolev spaces of sections of vector bundles can be found in Appendix A.4, based on Besov spaces and partitions of unity. The case of the sections of the trivial bundle of scalars can also be found in [19], and the case of tensors can also be found in [42]. Let $\nabla_{a}$ be the Levi-Civita connection associated with the metric $h_{a b}$, that is, the unique torsion-free connection satisfying $\nabla_{a} h_{b c}=0$. Let $R_{a b c}{ }^{d}$ be the Riemann tensor of the connection $\nabla_{a}$, where the sign convention used in this article is $\left(\nabla_{a} \nabla_{b}-\nabla_{b} \nabla_{a}\right) v_{c}=R_{a b c}{ }^{d} v_{d}$. Denote by $R_{a b}:=R_{a c b}{ }^{c}$ the Ricci tensor and by $R:=R_{a b} h^{a b}$ the Ricci scalar curvature of this connection.

Integration on $\mathcal{M}$ can be defined with the volume form associated with the metric $h_{a b}$. Given an arbitrary tensor $u^{a_{1} \cdots a_{r}} b_{1} \cdots b_{s}$ of type $m=r+s$, we define a real-valued function measuring its magnitude at any point $x \in \mathcal{M}$ as

$$
|u|:=\left(u^{a_{1} \cdots b_{s}} u_{a_{1} \cdots b_{s}}\right)^{1 / 2}
$$

A norm of an arbitrary tensor field $u^{a_{1} \cdots a_{r}} b_{1} \cdots b_{s}$ on $\mathcal{M}$ can then be defined for any $1 \leqslant p<\infty$ and for $p=\infty$ respectively using (2.1) as follows:

$$
\|u\|_{p}:=\left(\int_{\mathcal{M}}|u|^{p} d x\right)^{1 / p}, \quad\|u\|_{\infty}:=\text { ess } \sup _{x \in \mathcal{M}}|u| .
$$

One way to construct the Lebesgue spaces $L^{p}\left(T_{s}^{r} \mathcal{M}\right)$ of sections of the $(r, s)$-tensor bundle, for $1 \leqslant p \leqslant \infty$, is through the completion of $C^{\infty}\left(T_{s}^{r} \mathcal{M}\right)$ with respect to the $L^{p}$-norm (2.2). The $L^{p}$ spaces are Banach spaces, and the case $p=2$ is a Hilbert space with the inner product and norm given by

$$
(u, v):=\int_{\mathcal{M}} u_{a_{1} \cdots a_{m}} v^{a_{1} \cdots a_{m}} d x, \quad\|u\|:=\sqrt{(u, u)}=\|u\|_{2} .
$$

Denote covariant derivatives of tensor fields as $\nabla^{k} u^{a_{1} \cdots a_{m}}:=\nabla_{b_{1}} \cdots \nabla_{b_{k}} u^{a_{1} \cdots a_{m}}$, where $k$ denotes the total number of derivatives represented by the tensor indices $\left(b_{1}, \ldots, b_{k}\right)$. 
Another norm on $C^{\infty}\left(T_{s}^{r} \mathcal{M}\right)$ is given for any non-negative integer $k$ and for any $1 \leqslant p \leqslant \infty$ as follows:

$$
\|u\|_{k, p}:=\sum_{l=0}^{k}\left\|\nabla^{l} u\right\|_{p} .
$$

The Sobolev spaces $W^{k, p}\left(T_{s}^{r} \mathcal{M}\right)$ of sections of the $(r, s)$-tensor bundle can be defined as the completion of $C^{\infty}\left(T_{s}^{r} \mathcal{M}\right)$ with respect to the $W^{k, p}$-norm (2.4). The Sobolev spaces $W^{k, p}$ are Banach spaces, and the case $p=2$ is a Hilbert space. We have $L^{p}=W^{0, p}$ and $\|s\|_{p}=\|s\|_{0, p}$. See Appendix A.4 for a more careful construction that includes real order Sobolev spaces of sections of vector bundles.

Let $C_{+}^{\infty}$ be the set of nonnegative smooth (scalar) functions on $\mathcal{M}$. Then we can define order cone

$$
W_{+}^{s, p}:=\left\{\phi \in W^{s, p}:\langle\phi, \varphi\rangle \geqslant 0 \quad \forall \varphi \in C_{+}^{\infty}\right\}
$$

with respect to which the Sobolev spaces $W^{s, p}=W^{s, p}(\mathcal{M})$ are ordered Banach spaces. Here $\langle\cdot, \cdot\rangle$ is the unique extension of the $L^{2}$-inner product to a bilinear form $W^{s, p} \otimes$ $W^{-s, p^{\prime}} \rightarrow \mathbb{R}$, with $\frac{1}{p^{\prime}}+\frac{1}{p}=1$. The order relation is then $\phi \geqslant \psi$ iff $\phi-\psi \in W_{+}^{s, p}$. We note that this order cone is normal only for $s=0$. See Appendix A.2, where we review the main properties of ordered Banach spaces.

2.2. The Einstein constraint equations. We give a quick overview of the Einstein constraint equations in general relativity, and then define weak formulations that are fundamental to both solution theory and the development of approximation theory. Analogous material for the case of compact manifolds with boundary can be found in [21].

Let $\left(M, g_{\mu \nu}\right)$ be a 4-dimensional spacetime, that is, $M$ is a 4-dimensional, smooth manifold, and $g_{\mu \nu}$ is a smooth, Lorentzian metric on $M$ with signature $(-,+,+,+)$. Let $\nabla_{\mu}$ be the Levi-Civita connection associated with the metric $g_{\mu \nu}$. The Einstein equation is

$$
G_{\mu \nu}=\kappa T_{\mu \nu}
$$

where $G_{\mu \nu}=R_{\mu \nu}-\frac{1}{2} R g_{\mu \nu}$ is the Einstein tensor, $T_{\mu \nu}$ is the stress-energy tensor, and $\kappa=8 \pi G / c^{4}$, with $G$ the gravitation constant and $c$ the speed of light. The Ricci tensor is $R_{\mu \nu}=R_{\mu \sigma \nu}{ }^{\sigma}$ and $R=R_{\mu \nu} g^{\mu \nu}$ is the Ricci scalar, where $g^{\mu \nu}$ is the inverse of $g_{\mu \nu}$, that is $g_{\mu \sigma} g^{\sigma \nu}=\delta_{\mu}{ }^{\nu}$. The Riemann tensor is defined by $R_{\mu \nu \sigma}{ }^{\rho} w_{\rho}=\left(\nabla_{\mu} \nabla_{\nu}-\nabla_{\nu} \nabla_{\mu}\right) w_{\sigma}$, where $w_{\mu}$ is any 1 -form on $M$. The stress energy tensor $T_{\mu \nu}$ is assumed to be symmetric and to satisfy the condition $\nabla_{\mu} T^{\mu \nu}=0$ and the dominant energy condition, that is, the vector $-T^{\mu \nu} v_{v}$ is timelike and future-directed, where $v^{\mu}$ is any timelike and futuredirected vector field. In this section Greek indices $\mu, v, \sigma, \rho$ denote abstract spacetime indices, that is, tensorial character on the 4-dimensional manifold $M$. They are raised and lowered with $g^{\mu \nu}$ and $g_{\mu \nu}$, respectively. Latin indices $a, b, c, d$ will denote tensorial character on a 3-dimensional manifold.

The map $t: M \rightarrow \mathbb{R}$ is a time function iff the function $t$ is differentiable and the vector field $-\nabla^{\mu} t$ is a timelike, future-directed vector field on $M$. Introduce the hypersurface $\mathcal{M}:=\{x \in M: t(x)=0\}$, and denote by $n_{\mu}$ the unit 1-form orthogonal to $\mathcal{M}$. By definition of $\mathcal{M}$ the form $n_{\mu}$ can be expressed as $n_{\mu}=-\alpha \nabla_{\mu} t$, where $\alpha$, called the 
lapse function, is the positive function such that $n_{\mu} n_{v} g^{\mu \nu}=-1$. Let $\hat{h}_{\mu \nu}$ and $\hat{k}_{\mu \nu}$ be the first and second fundamental forms of $\mathcal{M}$, that is,

$$
\hat{h}_{\mu \nu}:=g_{\mu \nu}-n_{\mu} n_{\nu}, \quad \hat{k}_{\mu \nu}:=-\hat{h}_{\mu}^{\sigma} \nabla_{\sigma} n_{\nu} .
$$

The Einstein constraint equations on $\mathcal{M}$ are given by

$$
\left(G_{\mu \nu}-\kappa T_{\mu \nu}\right) n^{\nu}=0
$$

A well known calculation allows us to express these equations involving tensors on $M$ as equations involving intrinsic tensors on $\mathcal{M}$. The result is the following equations:

$$
\begin{aligned}
{ }^{3} \hat{R}+\hat{k}^{2}-\hat{k}_{a b} \hat{k}^{a b}-2 \kappa \hat{\rho} & =0, \\
\hat{D}^{a} \hat{k}-\hat{D}_{b} \hat{k}^{a b}+\kappa \hat{\jmath}^{a} & =0,
\end{aligned}
$$

where tensors $\hat{h}_{a b}, \hat{k}_{a b}, \hat{J}_{a}$ and $\hat{\rho}$ on a 3 -dimensional manifold are the pull-backs on $\mathcal{M}$ of the tensors $\hat{h}_{\mu \nu}, \hat{k}_{\mu \nu}, \hat{\jmath}_{\mu}$ and $\hat{\rho}$ on the 4-dimensional manifold $M$. We have introduced the energy density $\hat{\rho}:=n_{\mu} n_{\mu} T^{\mu \nu}$ and the momentum current density $\hat{\jmath}_{\mu}:=-\hat{h}_{\mu \nu} n_{\sigma} T^{v \sigma}$. We have denoted by $\hat{D}_{a}$ the Levi-Civita connection associated to $\hat{h}_{a b}$, so $\left(\mathcal{M}, \hat{h}_{a b}\right)$ is a 3-dimensional Riemannian manifold, with $\hat{h}_{a b}$ having signature $(+,+,+)$, and we use the notation $\hat{h}^{a b}$ for the inverse of the metric $\hat{h}_{a b}$. Indices have been raised and lowered with $\hat{h}^{a b}$ and $\hat{h}_{a b}$, respectively. We have also denoted by ${ }^{3} \hat{R}$ the Ricci scalar curvature of the metric $\hat{h}_{a b}$. Finally, recall that the constraint Eqs. (2.6)-(2.7) are indeed equations on $\hat{h}_{a b}$ and $\hat{k}_{a b}$ due to the matter fields satisfying the energy condition $-\hat{\rho}^{2}+\hat{\jmath}_{a} \hat{\jmath}^{a} \leqslant 0$ (with strict inequality holding at points on $\mathcal{M}$, where $\hat{\rho} \neq 0$; see [48]), which is implied by the dominant energy condition on the stress-energy tensor $T^{\mu \nu}$ in spacetime.

2.3. Conformal transverse traceless decomposition. Let $\phi$ denote a positive scalar field on $\mathcal{M}$, and decompose the extrinsic curvature tensor $\hat{k}_{a b}=\hat{l}_{a b}+\frac{1}{3} \hat{h}_{a b} \hat{\tau}$, where $\hat{\tau}:=$ $\hat{k}_{a b} \hat{h}^{a b}$ is the trace and then $\hat{l}_{a b}$ is the traceless part of the extrinsic curvature tensor. Then, introduce the following conformal re-scaling:

$$
\begin{aligned}
\hat{h}_{a b} & =: \phi^{4} h_{a b}, \quad \hat{l}^{a b}=: \phi^{-10} l^{a b}, \hat{\tau}=: \tau, \\
\hat{\jmath}^{a} & =: \phi^{-10} j^{a}, \quad \hat{\rho}=: \phi^{-8} \rho .
\end{aligned}
$$

We have introduced the Riemannian metric $h_{a b}$ on the 3-dimensional manifold $\mathcal{M}$, which determines the Levi-Civita connection $D_{a}$, and so we have that $D_{a} h_{b c}=0$. We have also introduced the symmetric, traceless tensor $l_{a b}$, and the non-physical matter sources $j^{a}$ and $\rho$. The different powers of the conformal re-scaling above are carefully chosen so that the constraint Eqs. (2.6)-(2.7) transform into the following equations:

$$
\begin{aligned}
& -8 \Delta \phi+{ }^{3} R \phi+\frac{2}{3} \tau^{2} \phi^{5}-l_{a b} l^{a b} \phi^{-7}-2 \kappa \rho \phi^{-3}=0, \\
& -D_{b} l^{a b}+\frac{2}{3} \phi^{6} D^{a} \tau+\kappa j^{a}=0
\end{aligned}
$$

where in the equation above, and from now on, indices of unhatted fields are raised and lowered with $h^{a b}$ and $h_{a b}$ respectively. We have also introduced the Laplace-Beltrami 
operator with respect to the metric $h_{a b}$, acting on smooth scalar fields; it is defined as follows:

$$
\Delta \phi:=h^{a b} D_{a} D_{b} \phi
$$

Equations (2.9)-(2.10) can be obtained by a straightforward albeit long computation. In order to perform this calculation it is useful to recall that both $\hat{D}_{a}$ and $D_{a}$ are connections on the manifold $\mathcal{M}$, and so they differ on a tensor field $C_{a b}{ }^{c}$, which can be computed explicitly in terms of $\phi$, and has the form

$$
C_{a b}{ }^{c}=4 \delta_{(a}^{c} D_{b)} \ln (\phi)-2 h_{a b} h^{c d} D_{d} \ln (\phi) .
$$

We remark that the power four on the re-scaling of the metric $\hat{h}_{a b}$ and $\mathcal{M}$ being 3-dimensional imply that ${ }^{3} \hat{R}=\phi^{-5}\left({ }^{3} R \phi-8 \Delta \phi\right)$, or in other words, that $\phi$ satisfies the Yamabe-type problem:

$$
-8 \Delta \phi+{ }^{3} R \phi-{ }^{3} \hat{R} \phi^{5}=0, \quad \phi>0,
$$

where ${ }^{3} \hat{R}$ represents the scalar curvature corresponding to the physical metric $\hat{h}_{a b}=$ $\phi^{4} h_{a b}$. Note that for any other power in the re-scaling, terms proportional to $h^{a b}\left(D_{a} \phi\right)$ $\left(D_{b} \phi\right) / \phi^{2}$ appear in the transformation. The set of all metrics on a closed manifold can be classified into the three disjoint Yamabe classes $\mathcal{Y}^{+}(\mathcal{M}), \mathcal{Y}^{0}(\mathcal{M})$, and $\mathcal{Y}^{-}(\mathcal{M})$, corresponding to whether one can conformally transform the metric into a metric with strictly positive, zero, or strictly negative scalar curvature, respectively, cf. [31] (see also Appendix A.7). We note that the Yamabe problem is to determine, for a given metric $h_{a b}$, whether there exists a conformal transformation $\phi$ solving (2.12) such that ${ }^{3} \hat{R}=$ const. Arguments similar to those above for $\phi$ force the power negative ten on the re-scaling of the tensor $\hat{l}^{a b}$ and $\hat{\jmath}^{a}$, so terms proportional to $\left(D_{a} \phi\right) / \phi$ cancel out in (2.10). Finally, the ratio between the conformal re-scaling powers of $\hat{\rho}$ and $\hat{j}^{a}$ is chosen such that the inequality $-\rho^{2}+h_{a b} j^{a} j^{b} \leqslant 0$ implies the inequality $-\hat{\rho}^{2}+\hat{h}_{a b} \hat{\jmath}^{a} \hat{\jmath}^{b} \leqslant 0$. For a complete discussion of all possible choices of re-scaling powers, see Appendix A.9.

There is one more step to convert the original constraint equation (2.6)-(2.7) into a determined elliptic system of equations. This step is the following: Decompose the symmetric, traceless tensor $l_{a b}$ into a divergence-free part $\sigma_{a b}$, and the symmetrized and traceless gradient of a vector, that is, $l^{a b}=: \sigma^{a b}+(\mathcal{L} w)^{a b}$, where $D_{a} \sigma^{a b}=0$ and we have introduced the conformal Killing operator $\mathcal{L}$ acting on smooth vector fields and defined as follows:

$$
(\mathcal{L} w)^{a b}:=D^{a} w^{b}+D^{b} w^{a}-\frac{2}{3}\left(D_{c} w^{c}\right) h^{a b} .
$$

Therefore, the constraint Eqs. (2.6)-(2.7) are transformed by the conformal re-scaling into the following equations:

$$
\begin{aligned}
& -8 \Delta \phi+{ }^{3} R \phi+\frac{2}{3} \tau^{2} \phi^{5}-\left[\sigma_{a b}+(\mathcal{L} w)_{a b}\right]\left[\sigma^{a b}+(\mathcal{L} w)^{a b}\right] \phi^{-7}-2 \kappa \rho \phi^{-3}=0, \\
& -D_{b}(\mathcal{L} w)^{a b}+\frac{2}{3} \phi^{6} D^{a} \tau+\kappa j^{a}=0 .
\end{aligned}
$$

In the next section we interpret these equations above as partial differential equations for the scalar field $\phi$ and the vector field $w^{a}$, while the rest of the fields are considered 
as given fields. Given a solution $\phi$ and $w^{a}$ of Eqs. (2.14)-(2.15), the physical metric $\hat{h}_{a b}$ and extrinsic curvature $\hat{k}^{a b}$ of the hypersurface $\mathcal{M}$ are given by

$$
\hat{h}_{a b}=\phi^{4} h_{a b}, \quad \hat{k}^{a b}=\phi^{-10}\left[\sigma^{a b}+(\mathcal{L} w)^{a b}\right]+\frac{1}{3} \phi^{-4} \tau h^{a b},
$$

while the matter fields are given by Eq (2.8).

From this point forward, for simplicity we will denote the Levi-Civita connection of the metric $h_{a b}$ on the 3-dimensional manifold $\mathcal{M}$ as $\nabla_{a}$ rather than $D_{a}$, and the Ricci scalar of $h_{a b}$ will be denoted by $R$ instead of ${ }^{3} R$. Let $(\mathcal{M}, h)$ be a 3-dimensional Riemannian manifold, where $\mathcal{M}$ is a smooth, compact manifold without boundary, and $h \in C^{\infty}\left(T_{2}^{0} \mathcal{M}\right)$ is a positive definite metric. With the shorthands $C^{\infty}=C^{\infty}(\mathcal{M} \times \mathbb{R})$ and $C^{\infty}=C^{\infty}(T \mathcal{M})$, let $L: C^{\infty} \rightarrow C^{\infty}$ and $\mathbb{L}: C^{\infty} \rightarrow C^{\infty}$ be the operators with actions on $\phi \in C^{\infty}$ and $\boldsymbol{w} \in \boldsymbol{C}^{\infty}$ given by

$$
\begin{aligned}
L \phi & :=-\Delta \phi, \\
\left(\mathbb{L}_{\boldsymbol{w}}\right)^{a} & :=-\nabla_{b}(\mathcal{L} \boldsymbol{w})^{a b},
\end{aligned}
$$

where $\Delta$ denotes the Laplace-Beltrami operator defined in (2.11), and where $\mathcal{L}$ denotes the conformal Killing operator defined in (2.13). We will also use the index-free notation $\mathbb{L} \boldsymbol{w}$ and $\mathcal{L} \boldsymbol{w}$.

The freely specifiable functions of the problem are a scalar function $\tau$, interpreted as the trace of the physical extrinsic curvature; a symmetric, traceless, and divergence-free, contravariant, two index tensor $\sigma$; the non-physical energy density $\rho$ and the non-physical momentum current density vector $\boldsymbol{j}$ subject to the requirement $-\rho^{2}+\boldsymbol{j} \cdot \boldsymbol{j} \leqslant 0$. The term non-physical refers here to a conformal rescaled field, while physical refers to a conformally non-rescaled term. The requirement on $\rho$ and $\boldsymbol{j}$ mentioned above and the particular conformal rescaling used in the semi-decoupled decomposition imply that the same inequality is satisfied by the physical energy and momentum current densities. This is a necessary condition (although not sufficient) in order that the matter sources in spacetime satisfy the dominant energy condition. The definition of various energy conditions can be found in [48, p. 219]. Introduce the non-linear operators $F: C^{\infty} \times C^{\infty} \rightarrow C^{\infty}$ and $\mathbb{F}: C^{\infty} \rightarrow C^{\infty}$ given by

$$
F(\phi, w)=a_{\tau} \phi^{5}+a_{R} \phi-a_{\rho} \phi^{-3}-a_{w} \phi^{-7}, \text { and } \mathbb{F}(\phi)=\boldsymbol{b}_{\tau} \phi^{6}+\boldsymbol{b}_{j},
$$

where the coefficient functions are defined as follows:

$$
\begin{array}{llrl}
a_{\tau}:=\frac{1}{12} \tau^{2}, & a_{R}:=\frac{1}{8} R, & a_{\rho}:=\frac{\kappa}{4} \rho, \\
a_{\boldsymbol{w}}:=\frac{1}{8}(\sigma+\mathcal{L} \boldsymbol{w})_{a b}(\sigma+\mathcal{L} \boldsymbol{w})^{a b}, & b_{\tau}^{a}:=\frac{2}{3} \nabla^{a} \tau, & b_{j}^{a}:=\kappa j^{a} .
\end{array}
$$

Notice that the scalar coefficients $a_{\tau}, a_{w}$, and $a_{\rho}$ are non-negative, while there is no sign restriction on $a_{R}$.

With these notations, the classical formulation (or the strong formulation) of the coupled Einstein constraint equations reads: Given the freely specifiable smooth functions $\tau, \sigma, \rho$, and $\boldsymbol{j}$ in $\mathcal{M}$, find a scalar field $\phi$ and a vector field $\boldsymbol{w}$ in $\mathcal{M}$ solution of the system

$$
L \phi+F(\phi, w)=0 \quad \text { and } \quad \mathbb{L} \boldsymbol{w}+\mathbb{F}(\phi)=0 \quad \text { in } \mathcal{M}
$$


2.4. Formulation in Sobolev spaces. We now outline a formulation of the Einstein constraint equations that involves the weakest regularity of the equation coefficients such that the equation itself is well-defined. So in particular, the operators $L$ and $\mathbb{L}$ are no longer differential operators sending smooth sections to smooth sections. We shall employ Sobolev spaces to quantify smoothness, cf. Appendix A.4.

Let $(\mathcal{M}, h)$ be a 3-dimensional Riemannian manifold, where $\mathcal{M}$ is a smooth, compact manifold without boundary, and with $p \in\left(\frac{3}{2}, \infty\right)$ and $s \in\left(\frac{3}{p}, \infty\right) \cap[1,2], h \in$ $W^{s, p}\left(T_{2}^{0} \mathcal{M}\right)$ is a positive definite metric. Note that the restriction $s \leqslant 2$ is only apparent, since $W^{t, p} \hookrightarrow W^{2, p}$ for any $t>2$. In the formulation of the constraint equations we need to distinguish the cases $s>2$ and $s \leqslant 2$ at least notation-wise, and we choose to present in this subsection the case $s \leqslant 2$ because this is the case that is considered in the core existence theory; the higher regularity is obtained by a standard bootstrapping technique. The general case is discussed in Sects. 4 and 6. Let us define $r=r(s, p)=\frac{3 p}{3+(2-s) p}$, so that the continuous embedding $L^{r} \hookrightarrow W^{s-2, p}$ holds. Introduce the operators

$$
A_{L}: W^{s, p} \rightarrow W^{s-2, p}, \quad \text { and } \quad A_{\mathbb{L}}: W^{1,2 r} \rightarrow W^{-1,2 r},
$$

as the unique extensions of the operators $L$ and $\mathbb{L}$ in Eqs. (2.16) and (2.17), respectively, cf. Lemma 31 in Appendix A.5. The boldface letters denote spaces of sections of the tangent bundle $T \mathcal{M}$, e.g., $\boldsymbol{W}^{1,2 r}=W^{1,2 r}(T \mathcal{M})$.

Fix the source functions

$$
\tau \in L^{2 r}, \quad \rho \in W_{+}^{s-2, p}, \quad \sigma \in L^{2 r}, \quad \boldsymbol{j} \in \boldsymbol{W}^{-1,2 r},
$$

where $\sigma$ is symmetric, traceless and divergence-free in the weak sense, the latter meaning that $\langle\sigma, \mathcal{L} \boldsymbol{\omega}\rangle=0$ for all $\boldsymbol{\omega} \in \boldsymbol{W}^{1,(2 r)^{\prime}}$. Here $\frac{1}{(2 r)^{\prime}}+\frac{1}{2 r}=1$, and $\langle\cdot, \cdot\rangle$ denotes the extension of the $L^{2}$-inner product to $\boldsymbol{W}^{-1,2 r} \otimes \boldsymbol{W}^{1,(2 r)^{\prime}}$. We say that the matter fields $\rho$ and $\boldsymbol{j}$ satisfy the energy condition iff there exist sequences $\left\{\rho_{n}\right\} \subset C^{\infty}$ and $\left\{\boldsymbol{j}_{n}\right\} \subset \boldsymbol{C}^{\infty}$, respectively converging to $\rho$ and $\boldsymbol{j}$ in the appropriate topology, such that

$$
\rho_{n}^{2}-\boldsymbol{j}_{n} \cdot \boldsymbol{j}_{n} \geqslant 0 \text {. }
$$

Given any function $\tau \in L^{2 r}$ we have $\boldsymbol{b}_{\tau} \equiv \frac{2}{3} \nabla \tau \in \boldsymbol{W}^{-1,2 r}$. The assumptions $\tau \in L^{2 r}$ and $\sigma \in L^{2 r}$ imply that for every $\boldsymbol{w} \in \boldsymbol{W}^{1,2 r}$ the functions $a_{\tau}$ and $a_{\boldsymbol{w}}$ belong to $L^{r}$. For example, to see that $a_{w} \in L^{r}$, we proceed as

$$
\left\|a_{\boldsymbol{w}}\right\|_{r}=\|\sigma+\mathcal{L} \boldsymbol{w}\|_{2 r} \leqslant 2\left(\|\sigma\|_{2 r}^{2}+\|\mathcal{L} \boldsymbol{w}\|_{2 r}^{2}\right) \leqslant 2\left(\|\sigma\|_{2 r}^{2}+c_{\mathcal{L}}^{2}\|\boldsymbol{w}\|_{1,2 r}^{2}\right),
$$

where we used the boundedness $\|\mathcal{L} \boldsymbol{w}\|_{2 r} \leqslant c_{\mathcal{L}}\|\boldsymbol{w}\|_{1,2 r}$. The assumption on the background metric implies that $a_{R} \in W^{s-2, p}$.

Given any two functions $u, v \in L^{\infty}$, and $t \geqslant 0$ and $q \in[1, \infty]$, define the interval

$$
[u, v]_{t, q}:=\left\{\phi \in W^{t, q}: u \leqslant \phi \leqslant v\right\} \subset W^{t, q},
$$

see Lemma 1 near the end of Sect. 3. We equip $[u, v]_{t, q}$ with the subspace topology of $W^{t, q}$. We will write $[u, v]_{q}$ for $[u, v]_{0, q}$, and $[u, v]$ for $[u, v]_{\infty}$. Now, assuming that $\phi_{-}, \phi_{+} \in W^{s, p}$ and $0<\phi_{-} \leqslant \phi_{+}<\infty$, we introduce the non-linear operators

$$
f:\left[\phi_{-}, \phi_{+}\right]_{s, p} \times \boldsymbol{W}^{1,2 r} \rightarrow W^{s-2, p}, \quad \text { and } \quad f:\left[\phi_{-}, \phi_{+}\right]_{s, p} \rightarrow \boldsymbol{W}^{-1,2 r},
$$


by

$$
f(\phi, w)=a_{\tau} \phi^{5}+a_{R} \phi-a_{\rho} \phi^{-3}-a_{\boldsymbol{w}} \phi^{-7}, \quad \text { and } \boldsymbol{f}(\phi)=\boldsymbol{b}_{\tau} \phi^{6}+\boldsymbol{b}_{j},
$$

where the pointwise multiplication by an element of $W^{s, p}$ defines a bounded linear map in $W^{s-2, p}$ and in $\boldsymbol{W}^{-1,2 r}$, cf. Corollary 3(a) in Appendix A.4.

Now, we can formulate the Einstein constraint equations in terms of the above defined operators: Find elements $\phi \in\left[\phi_{-}, \phi_{+}\right]_{s, p}$ and $\boldsymbol{w} \in \boldsymbol{W}^{1,2 r}$ solutions of

$$
\begin{aligned}
A_{L} \phi+f(\phi, w) & =0, \\
A_{\mathbb{L}} \boldsymbol{w}+\boldsymbol{f}(\phi) & =0 .
\end{aligned}
$$

In the following, often we treat the two equations separately. The Hamiltonian constraint equation is the following: Given a function $\boldsymbol{w} \in \boldsymbol{W}^{1,2 r}$, find an element $\phi \in\left[\phi_{-}, \phi_{+}\right]_{s, p}$ solution of

$$
A_{L} \phi+f(\phi, w)=0
$$

When the Hamiltonian constraint equation is under consideration, the function $\boldsymbol{w}$ is referred to as the source. To indicate the dependence of the solution $\phi$ on the source $\boldsymbol{w}$, sometimes we write $\phi=\phi_{w}$. Let us define the momentum constraint equation: Given $\phi \in W^{s, p}$ with $\phi>0$, find an element $\boldsymbol{w} \in \boldsymbol{W}^{1,2 r}$ solution of

$$
A_{\mathbb{L}} \boldsymbol{w}+\boldsymbol{f}(\phi)=0 .
$$

When the momentum constraint equation is under consideration, the function $\phi$ is referred to as the source. To indicate the dependence of the solution $\boldsymbol{w}$ on the source $\phi$, sometimes we write $\boldsymbol{w}=\boldsymbol{w}_{\phi}$.

\section{Overview of the Main Results}

In this section, we state our three main theorems (Theorems 1, 2, and 3 below) on the existence of far-from-CMC, near-CMC, and CMC solutions to the Einstein constraint equations, and give an outline of the overall structure of the argument that we build in the paper. The proofs of the main results appear in Sect. 6 toward the end of the paper, after we develop a number of supporting results in the body of the paper. After we give an overview of the basic abstract structure of the coupled nonlinear constraint problem, we prove two abstract topological fixed-point theorems (Theorems 4 and 5) that are the basis for our analysis of the coupled system; these arguments are also the basis for our results in [21] on existence of non-CMC solutions to the Einstein constraints on compact manifolds with boundary. After proving these abstract results, we give an overview of the technical results that must be established in the remainder of the paper in order to use the abstract results.

Before stating the main theorems, let us make precise what we mean by near-CMC condition in this article. We say that the extrinsic mean curvature $\tau$ satisfies the nearCMC condition when the following inequality is satisfied:

$$
\|\nabla \tau\|_{z}<\Gamma \inf _{\mathcal{M}}|\tau|
$$

where the constant $\Gamma=\frac{\sqrt{3}}{2 C}$ if $\rho, \sigma^{2} \in L^{\infty}$, and $\Gamma=\frac{\sqrt{3}}{2 C}\left(\frac{\min u v}{\max u v}\right)^{6}$ otherwise, with the constant $C>0$ as in Corollary 1 and the continuous functions $u, v>0$ are as defined in 
(5.14) or in (5.15) on page. Here $C$ depends only on the Riemannian manifold $\left(\mathcal{M}, h_{a b}\right)$, and not mentioning $\left(\mathcal{M}, h_{a b}\right), u$ and $v$ depend only on $\rho, \sigma^{2}$, and $\tau$. It is important to note that we always have $0<\frac{\min u v}{\max u v} \leqslant 1$, so that in any case the condition (3.1) is at least as strong as the same condition with $\Gamma$ taken to be equal to $\frac{\sqrt{3}}{2 C}$. The condition depends on the value of $z$, and that will be inserted through the context.

Recall that the three Yamabe classes $\mathcal{Y}^{+}(\mathcal{M}), \mathcal{Y}^{-}(\mathcal{M})$ and $\mathcal{Y}^{0}(\mathcal{M})$ are defined after Eq. (2.12). See Appendix A.7 for more details.

3.1. Theorem 1: Far-CMC weak solutions. Here is the first of our three main results. This result does not involve the near-CMC condition, which is one of the main contributions of this paper. The result is developed in the presence of a weak background metric $h_{a b} \in W^{s, p}$, for $p \in(1, \infty)$ and $s \in\left(1+\frac{3}{p}, \infty\right)$, with the weakest possible assumptions on the data that allows for avoiding the near-CMC condition.

Theorem 1 (Far-CMC $W^{s, p}$ solutions, $\left.p \in(1, \infty), s \in\left(1+\frac{3}{p}, \infty\right)\right)$. Let $\left(\mathcal{M}, h_{a b}\right)$ be a 3-dimensional closed Riemannian manifold. Let $h_{a b} \in W^{s, p}$ admit no conformal Killing field and be in $\mathcal{Y}^{+}(\mathcal{M})$, where $p \in(1, \infty)$ and $s \in\left(1+\frac{3}{p}, \infty\right)$ are given. Select $q$ and e to satisfy:

- $\frac{1}{q} \in(0,1) \cap\left(0, \frac{s-1}{3}\right) \cap\left[\frac{3-p}{3 p}, \frac{3+p}{3 p}\right]$,

- $e \in\left(1+\frac{3}{q}, \infty\right) \cap[s-1, s] \cap\left[\frac{3}{q}+s-\frac{3}{p}-1, \frac{3}{q}+s-\frac{3}{p}\right]$.

Assume that the data satisfies:

- $\tau \in W^{e-1, q}$ if $e \geqslant 2$, and $\tau \in W^{1, z}$ otherwise, with $z=\frac{3 q}{3+\max \{0,2-e\} q}$,

- $\sigma \in W^{e-1, q}$, with $\left\|\sigma^{2}\right\|_{\infty}$ sufficiently small,

- $\rho \in W_{+}^{s-2, p} \cap L^{\infty} \backslash\{0\}$, with $\|\rho\|_{\infty}$ sufficiently small,

- $\boldsymbol{j} \in \boldsymbol{W}^{e-2, q}$, with $\|\boldsymbol{j}\|_{e-2, q}$ sufficiently small.

Then there exist $\phi \in W^{s, p}$ with $\phi>0$ and $\boldsymbol{w} \in \boldsymbol{W}^{e, q}$ solving the Einstein constraint equations.

Proof. The proof will be given in Sect. 6. See Fig. 1 for clarification of the conditions on $e$ and $q$.

Remark 1. The above result avoids the near-CMC condition (3.1); however, one should be aware of the various smallness conditions involved in the above theorem. More precisely, the mean curvature $\tau$ can be chosen to be an arbitrary function from a suitable function space, and afterwards, one has to choose $\sigma, \rho$, and $\boldsymbol{j}$ satisfying smallness conditions that depend on the chosen $\tau$. Nevertheless, the novelty of this result is that $\tau$ can be specified freely, whereas the condition (3.1) is not satisfied for arbitrary $\tau$.

3.2. Theorem 2: Near-CMC weak solutions. Here is the second of our three main results; this result requires the near-CMC condition, but still extends the known near-CMC results to situations with weaker assumptions on metric and on the data. In particular, the result is developed in the presence of a weak background metric $h_{a b} \in W^{s, p}$, for $p \in(1, \infty)$ and $s \in\left(1+\frac{3}{p}, \infty\right)$, and with the weakest possible assumptions on the data.

Theorem 2 (Near-CMC $W^{s, p}$ solutions, $\left.p \in(1, \infty), s \in\left(1+\frac{3}{p}, \infty\right)\right)$. Let $\left(\mathcal{M}, h_{a b}\right)$ be a 3-dimensional closed Riemannian manifold. Let $h_{a b} \in W^{s, p}$ admit no conformal Killing field, where $p \in(1, \infty)$ and $s \in\left(1+\frac{3}{p}, \infty\right)$ are given. Select $q$, e and $z$ to satisfy: 


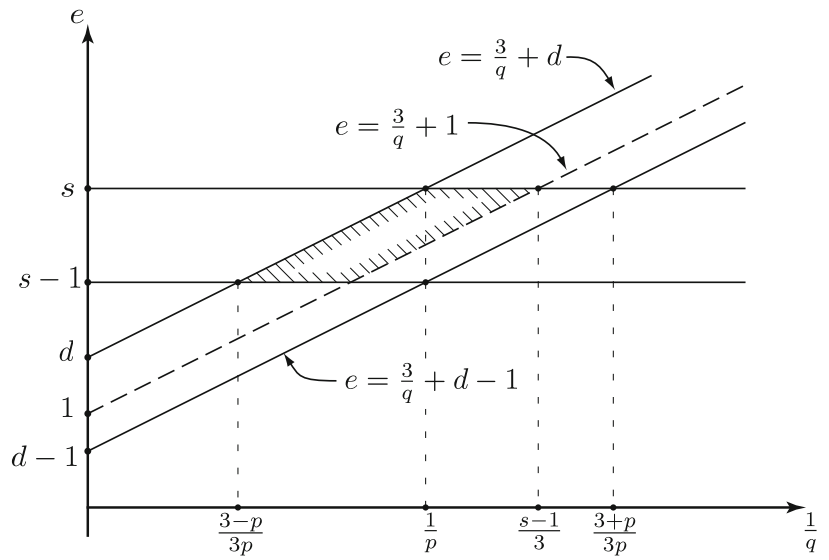

Fig. 1. Range of $e$ and $q$ in Theorems 1 and 2, with $d=s-\frac{3}{p}>1$

- $\frac{1}{q} \in(0,1) \cap\left(0, \frac{s-1}{3}\right) \cap\left[\frac{3-p}{3 p}, \frac{3+p}{3 p}\right]$.

- $e \in\left(1+\frac{3}{q}, \infty\right) \cap[s-1, s] \cap\left[\frac{3}{q}+s-\frac{3}{p}-1, \frac{3}{q}+s-\frac{3}{p}\right]$.

- $z=\frac{3 q}{3+\max \{0,2-e\} q}$.

Assume that $\tau$ satisfies the near-CMC condition (3.1) with $z$ as above, and that the data satisfies:

- $\tau \in W^{e-1, q}$ if $e>2$, and $\tau \in W^{1, z}$ if $e \leqslant 2$,

- $\sigma \in W^{e-1, q}$,

- $\rho \in W_{+}^{s-2, p}$,

- $\boldsymbol{j} \in \boldsymbol{W}^{e-2, q}$.

In addition, let one of the following sets of conditions hold:

(a) $h_{a b}$ is in $\mathcal{Y}^{-}(\mathcal{M})$; the metric $h_{a b}$ is conformally equivalent to a metric with scalar curvature $\left(-\tau^{2}\right)$;

(b) $h_{a b}$ is in $\mathcal{Y}^{0}(\mathcal{M})$ or in $\mathcal{Y}^{+}(\mathcal{M})$; either $\rho \not \equiv 0$ and $\tau \not \equiv 0$ or $\tau \in L^{\infty}$ and $\inf \mathcal{M}^{2}$ is sufficiently large.

Then there exist $\phi \in W^{s, p}$ with $\phi>0$ and $\boldsymbol{w} \in \boldsymbol{W}^{e, q}$ solving the Einstein constraint equations.

Proof. The proof will be given in Sect. 6. See Fig. 1 for clarification of the conditions on $e$ and $q$.

3.3. Theorem 3: CMC weak solutions. Here is the last of our three main results; it covers specifically the CMC case, and allows for lower regularity of the background metric than the non-CMC case. In particular, the result is developed with a weak background metric $h_{a b} \in W^{s, p}$, for $p \in(1, \infty)$ and $s \in\left(\frac{3}{p}, \infty\right) \cap[1, \infty)$. In the case of $s=2$, we reproduce the CMC existence results of Choquet-Bruhat [10], and in the case $p=2$, we reproduce the CMC existence results of Maxwell [33], but with a different proof; our CMC proof goes through the same analysis framework that we use to obtain the non-CMC results (Theorems 4 and 5). 
Theorem 3 (CMC $W^{s, p}$ solutions, $\left.p \in(1, \infty), s \in\left(\frac{3}{p}, \infty\right) \cap[1, \infty)\right)$. Let $\left(\mathcal{M}, h_{a b}\right)$ be a 3-dimensional closed Riemannian manifold. Let $h_{a b} \in W^{s, p}$ admit no conformal Killing field, where $p \in(1, \infty)$ and $s \in\left(\frac{3}{p}, \infty\right) \cap[1, \infty)$ are given. With $d:=s-\frac{3}{p}$, select $q$ and $e$ to satisfy:

- $\frac{1}{q} \in(0,1) \cap\left[\frac{3-p}{3 p}, \frac{3+p}{3 p}\right] \cap\left[\frac{1-d}{3}, \frac{3+s p}{6 p}\right)$,

- $e \in[1, \infty) \cap[s-1, s] \cap\left[\frac{3}{q}+d-1, \frac{3}{q}+d\right] \cap\left(\frac{3}{q}+\frac{d}{2}, \infty\right)$.

Assume $\tau=$ const $(C M C)$ and that the data satisfies:

- $\sigma \in W^{e-1, q}$,

- $\rho \in W_{+}^{s-2, p}$,

- $\boldsymbol{j} \in \boldsymbol{W}^{e^{-2}, q}$.

In addition, let one of the following sets of conditions hold:

(a) $h_{a b}$ is in $\mathcal{Y}^{-}(\mathcal{M}) ; \tau \neq 0$;

(b) $h_{a b}$ is in $\mathcal{Y}^{+}(\mathcal{M}) ; \rho \neq 0$ or $\sigma \neq 0$;

(c) $h_{a b}$ is in $\mathcal{Y}^{0}(\mathcal{M}) ; \tau \neq 0 ; \rho \neq 0$ or $\sigma \neq 0$;

(d) $h_{a b}$ is in $\mathcal{Y}^{0}(\mathcal{M}) ; \tau=\rho=\sigma=0 ; \boldsymbol{j}=0$.

Then there exist $\phi \in W^{s, p}$ with $\phi>0$ and $\boldsymbol{w} \in W^{e, q}$ solving the Einstein constraint equations.

Proof. The proof will be given in Sect. 6. See Fig. 2 for clarification of the conditions on $e$ and $q$.

3.4. A coupled topological fixed-point argument. In Theorems 4 and 5 below (see also [21]) we give some abstract fixed-point results which form the basic framework for our analysis of the coupled constraints. These topological fixed-point theorems will be the main tool by which we shall establish Theorems 1,2, and 3 above. They have the important feature that the required properties of the abstract fixed-point operators $S$ and $T$ appearing in Theorems 4 and 5 below can be established in the case of the Einstein

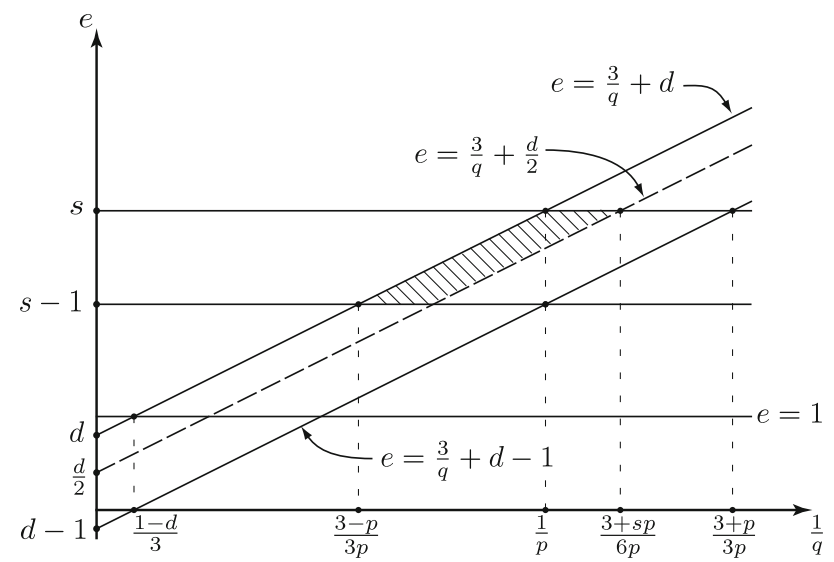

Fig. 2. Range of $e$ and $q$ in Theorem 3. Recall that $d=s-\frac{3}{p}>0$ 
constraints without using the near-CMC condition; this is not the case for fixed-point arguments for the constraints based on $k$-contractions (cf. [1,26]) which require nearCMC conditions. The bulk of the paper then involves establishing the required properties of $S$ and $T$ without using the near-CMC condition, and finding suitable global barriers $\phi_{-}$and $\phi_{+}$for defining the required set $U$ that are similarly free of the near-CMC condition (when possible).

We now set up the basic abstract framework. Let $X$ and $Y$ be Banach spaces, let $f: X \times Y \rightarrow X^{*}$ and $f: X \rightarrow Y^{*}$ be (generally nonlinear) operators, let $A_{\mathbb{L}}: Y \rightarrow Y^{*}$ be a linear invertible operator, and let $A_{L}: X \rightarrow X^{*}$ be a linear invertible operator satisfying the maximum principle, meaning that $A_{L} u \leqslant A_{L} v \Rightarrow u \leqslant v$. The order structure on $X$ for interpreting the maximum principle will be inherited from an ordered Banach space $Z$ (see Appendices A.2, A.3, and A.6, and also cf. [54]) through the compact embedding $X \hookrightarrow Z$, which will also make available compactness arguments.

The coupled Hamiltonian and momentum constraints can be viewed abstractly as coupled operator equations of the form:

$$
\begin{aligned}
A_{L} \phi+f(\phi, w) & =0, \\
A_{\mathbb{L}} w+f(\phi) & =0,
\end{aligned}
$$

or equivalently as the coupled fixed-point equations

$$
\begin{aligned}
\phi & =T(\phi, w), \\
w & =S(\phi),
\end{aligned}
$$

for appropriately defined fixed-point maps $T: X \times Y \rightarrow X$ and $S: X \rightarrow Y$. The obvious choice for $S$ is the Picard map for (3.3),

$$
S(\phi)=-A_{\mathbb{L}}^{-1} \boldsymbol{f}(\phi),
$$

which also happens to be the solution map for (3.3). On the other hand, there are a number of distinct possibilities for $T$, ranging from the solution map for (3.2), to the Picard map for (3.2), which inverts only the linear part of the operator in (3.2):

$$
T(\phi, w)=-A_{L}^{-1} f(\phi, w) .
$$

Assume now that $T$ is as in (3.7), and (for fixed $w \in Y$ ) that $\phi_{-}$and $\phi_{+}$are sub- and super-solutions of the semi-linear operator equation (3.2) in the sense that

$$
A_{L} \phi_{-}+f\left(\phi_{-}, w\right) \leqslant 0, \quad A_{L} \phi_{+}+f\left(\phi_{+}, w\right) \geqslant 0 .
$$

The assumptions on $A_{L}$ imply (see Lemma 26 in Appendix A.3) that for fixed $w \in Y$, $\phi_{-}$and $\phi_{+}$are also sub- and super-solutions of the equivalent fixed-point equation:

$$
\phi_{-} \leqslant T\left(\phi_{-}, w\right), \quad \phi_{+} \geqslant T\left(\phi_{+}, w\right) .
$$

For developing results on fixed-point iterations in ordered Banach spaces, it is convenient to work with maps which are monotone increasing in $\phi$, for fixed $w \in Y$ :

$$
\phi_{1} \leqslant \phi_{2} \quad \Longrightarrow \quad T\left(\phi_{1}, w\right) \leqslant T\left(\phi_{2}, w\right) \text {. }
$$

The map $T$ that arises as the Picard map for a semi-linear problem will generally not be monotone increasing; however, if there exists a continuous linear monotone increasing 
map $J: X \rightarrow X^{*}$, then one can always introduce a positive shift $s$ into the operator equation

$$
A_{L}^{s} \phi+f^{s}(\phi, w)=0
$$

with $A_{L}^{s}=A_{L}+s J$ and $f^{s}(\phi, w)=f(\phi, w)-s J \phi$. (Throughout this paper, the spaces we encounter for $X$ typically fit into a Gelfand triple $X \hookrightarrow H \hookrightarrow X^{*}$, where the "pivot" space $H$ is Hilbert space, and the continuous map between $X$ and $X^{*}$ is a composition of the two inclusion maps.) Since $s>0$ the shifted operator $A_{L}^{S}$ retains the maximum principle property of $A_{L}$, and if $s$ is chosen sufficiently large then $f^{s}$ is monotone decreasing in $\phi$. Under the additional condition on $J$ and $s$ that $A_{L}^{s}$ is invertible (see also [21]), the shifted Picard map

$$
T^{s}(\phi, w)=-\left(A_{L}^{s}\right)^{-1} f^{s}(\phi, w)
$$

is now monotone increasing in $\phi$.

We now give two abstract existence results for systems of the form (3.4)-(3.5).

Theorem 4 (Coupled Fixed-Point Principle A). Let $X$ and $Y$ be Banach spaces, and let $Z$ be a Banach space with compact embedding $X \hookrightarrow Z$. Let $U \subset Z$ be a non-empty, convex, closed, bounded subset, and let

$$
S: U \rightarrow \mathcal{R}(S) \subset Y, \quad T: U \times \mathcal{R}(S) \rightarrow U \cap X,
$$

be continuous maps. Then there exist $\phi \in U \cap X$ and $w \in \mathcal{R}(S)$ such that

$$
\phi=T(\phi, w) \text { and } w=S(\phi) .
$$

Proof. The proof will be through a standard variation of the Schauder Fixed-Point Theorem, reviewed as Theorem 9 in Appendix A.1. The proof is divided into several steps.

Step 1. Construction of a non-empty, convex, closed, bounded subset $U \subset Z$. By assumption we have that $U \subset Z$ is non-empty, convex (involving the vector space structure of $Z$ ), closed (involving the topology on $Z$ ), and bounded (involving the metric given by the norm on $Z$ ).

Step 2. Continuity of a mapping $G: U \subset Z \rightarrow U \cap X \subset X$. Define the composite operator

$$
G:=T \circ S: U \subset Z \rightarrow U \cap X \subset X
$$

The mapping $G$ is continuous, since it is a composition of the continuous operators $S: U \subset Z \rightarrow \mathcal{R}(S) \subset Y$ and $T: U \subset Z \times \mathcal{R}(S) \rightarrow U \cap X \subset X$.

Step 3. Compactness of a mapping $F: U \subset Z \rightarrow U \subset Z$. The compact embedding assumption $X \hookrightarrow Z$ implies that the canonical injection operator $i: X \rightarrow Z$ is compact. Since the composition of compact and continuous operators is compact, we have the composition $F:=i \circ G: U \subset Z \rightarrow U \subset Z$ is compact.

Step 4. Invoking the Schauder Theorem. Therefore, by a standard variant of the Schauder Theorem (see Theorem 9 in Appendix A.1), there exists a fixed-point $\phi \in U$ such that $\phi=F(\phi)=T(\phi, S(\phi))$. Since $\mathcal{R}(T)=U \cap X$, in fact $\phi \in U \cap X$. We now take $w=S(\phi) \subset \mathcal{R}(S)$ and we have the result. 
The assumption in Theorem 4 that the mapping $T$ is invariant on the non-empty, closed, convex, bounded subset $U$ can be established using a priori estimates if $T$ is the solution mapping, but if there are multiple fixed-points then continuity of $T$ will not hold. Fixed-point theory for set-valued maps could still potentially be used (cf. [54]). On the other hand, if $T$ is chosen to be the Picard map, then it is typically easier to establish continuity of $T$ even with multiple fixed-points, but more difficult to establish the invariance property without additional conditions on $T$. In our setting, we wish to allow for non-uniqueness in the Hamiltonian constraint (for example see [21] for possible non-uniqueness in the case of compact manifolds with boundary), so will generally focus on the Picard map for the Hamiltonian constraint in our fixed-point framework for the coupled constraints. The following special case of Theorem 4 gives some simple sufficient conditions on $T$ to establish the invariance using barriers in an ordered Banach space (for a review of ordered Banach spaces, see Appendix A.2 or [54]).

Theorem 5 (Coupled Fixed-Point Principle B). Let $X$ and $Y$ be Banach spaces, and let $Z$ be a real ordered Banach space having the compact embedding $X \hookrightarrow Z$. Let $\left[\phi_{-}, \phi_{+}\right] \subset Z$ be a nonempty interval which is closed in the topology of $Z$, and set $U=\left[\phi_{-}, \phi_{+}\right] \cap \bar{B}_{M} \subset Z$, where $\bar{B}_{M}$ is the closed ball of finite radius $M>0$ in $Z$ about the origin. Assume $U$ is nonempty, and let the maps

$$
S: U \rightarrow \mathcal{R}(S) \subset Y, \quad T: U \times \mathcal{R}(S) \rightarrow U \cap X,
$$

be continuous maps. Then there exist $\phi \in U \cap X$ and $w \in \mathcal{R}(S)$ such that

$$
\phi=T(\phi, w) \text { and } w=S(\phi) .
$$

Proof. By choosing the set $U$ to be the non-empty intersection of the interval $\left[\phi_{-}, \phi_{+}\right]$ with a bounded set in $Z$, we have $U$ bounded in $Z$. We also have that $U$ is convex with respect to the vector space structure of $Z$, since it is the intersection of two convex sets $\left[\phi_{-}, \phi_{+}\right]$and $\bar{B}_{M}$. Since $U$ is the intersection of the interval $\left[\phi_{-}, \phi_{+}\right]$, which by assumption is closed in the topology of $Z$, with the closed ball $\bar{B}_{M}$ in $Z, U$ is also closed. In summary, we have that $U$ is non-empty as a subset of $Z$, closed in the topology of $Z$, convex with respect to the vector space structure of $Z$, and bounded with respect to the metric (via normed) space structure of $Z$. Therefore, the assumptions of Theorem 4 hold and the result then follows.

We make some final remarks about Theorems 4 and 5. If the ordered Banach space $Z$ in Theorem 5 had a normal order cone, then the closed interval $\left[\phi_{-}, \phi_{+}\right]$would automatically be bounded in the norm of $Z$ (see Lemma 20 in Appendix A.2 or [54] for this result). The interval by itself is also non-empty and closed by assumption, and trivially convex (see Appendix A.2), so that Theorem 5 would follow immediately from Theorem 4 by simply taking $U=\left[\phi_{-}, \phi_{+}\right]$. Second, the closed ball $\bar{B}_{M}$ in Theorem 5 can be replaced with any non-empty, convex, closed, and bounded subset of $Z$ having non-trivial intersection with the interval $\left[\phi_{-}, \phi_{+}\right]$. Third, in the case that $T$ in Theorem 5 arises as the Picard map (3.7) of the semi-linear problem (3.2), we can always ensure that $T$ is invariant on $U$ in Theorem 5 by: (1) obtaining sub- and super-solutions to the semi-linear operator equation and using these for $\phi_{-}$and $\phi_{+}$, since these will also be suband super-solutions for the fixed-point equation involving the Picard map; (2) introducing a shift into the nonlinearity to ensure $T$ is monotone increasing; and (3) obtaining $a$ priori norm bounds on Picard iterates. As noted earlier, (1) and (2) will ensure

$$
\phi_{-} \leqslant T\left(\phi_{-}, w\right) \leqslant T(\phi, w) \leqslant T\left(\phi_{+}, w\right) \leqslant \phi_{+},
$$


for all $\phi \in\left[\phi_{-}, \phi_{+}\right]$, and $w \in \mathcal{R}(S)$, whereas (3) ensures that

$$
\|T(\phi, w)\|_{X} \leqslant M, \quad \forall \phi \in\left[\phi_{-}, \phi_{+}\right], \quad \forall w \in \mathcal{R}(S),
$$

which together ensure $T: U \times \mathcal{R}(S) \rightarrow U \cap X$, where $U=\left[\phi_{-}, \phi_{+}\right] \cap \bar{B}_{M} \subset Z$. Again, if $Z$ has a normal order cone structure, then ensuring (3.8) holds will automatically guarantee that (3.9) also holds, so it is not necessary to establish (3.9) separately in the case of a normal order cone.

Finally, note that Theorem 5 also allows one to choose the solution map (or any other fixed-point map) for $T$ together with a priori order cone and norm estimates to ensure the conditions (3.8) and (3.9) hold (as long as continuity for $T$ can be shown). Even if a priori order-cone estimates cannot be shown to hold directly for this choice of $T$, as long as the map can be "bracketed" in the interval $\left[\phi_{-}, \phi_{+}\right]$by two auxiliary monotone increasing maps, then it can be shown that (3.8) holds. This allows one to use the Picard map even if it is not monotone increasing, without having to introduce the shift into the Picard map.

The overall argument we use to prove the non-CMC results in Theorems 1, 2, and 3 using Theorems 4 and 5 involves the following steps:

Step 1. The choice of function spaces. We will choose the spaces for use of Theorem 5 as follows:

- $X=W^{s, p}$, with $p \in(1, \infty)$, and $s(p) \in\left(1+\frac{3}{p}, \infty\right)$. In the CMC case in Theorem 3, we can lower $s$ to $s(p) \in\left(\frac{3}{p}, \infty\right) \cap[1, \infty)$.

- $Y=W^{e, q}$, with $e$ and $q$ as given in the theorem statements.

- $Z=W^{\tilde{s}, p}, \tilde{s} \in\left(\frac{3}{p}, s\right)$, so that $X=W^{s, p} \hookrightarrow W^{\tilde{s}, p}=Z$ is compact.

- $U=\left[\phi_{-}, \phi_{+}\right]_{\tilde{s}, p} \cap \bar{B}_{M} \subset W^{\tilde{s}, p}=Z$, with $\phi_{-}$and $\phi_{+}$global barriers (suband super-solutions, respectively) for the Hamiltonian constraint equation which satisfy the compatibility condition: $0<\phi_{-} \leqslant \phi_{+}<\infty$.

Step 2. Construction of the mapping S. Assuming the existence of "global" weak suband super-solutions $\phi_{-}$and $\phi_{+}$, and assuming the fixed function $\phi \in U=$ $\left[\phi_{-}, \phi_{+}\right]_{\tilde{s}, p} \cap \bar{B}_{M} \subset W^{\tilde{s}, p}=Z$ is taken as data in the momentum constraint, we establish continuity and related properties of the momentum constraint solution map $S: U \rightarrow \mathcal{R}(S) \subset W^{e, q}=Y$ (Sect. 4.1).

Step 3. Construction of the mapping T. Again assuming existence of "global" weak sub- and super-solutions $\phi_{-}$and $\phi_{+}$, with fixed $w \in \mathcal{R}(S) \subset \boldsymbol{W}^{e, q}=Y$ taken as data in the Hamiltonian constraint, we establish continuity and related properties of the Picard map $T: U \times \mathcal{R}(S) \rightarrow U \cap W^{s, p}$. Invariance of $T$ on $U=\left[\phi_{-}, \phi_{+}\right]_{\tilde{s}, p} \cap \bar{B}_{M} \subset W^{\tilde{s}, p}$ is established using a combination of a priori order cone bounds and norm bounds (Sect. 4.2).

Step 4. Barrier construction. Global weak sub- and super-solutions $\phi_{-}$and $\phi_{+}$for the Hamiltonian constraint are explicitly constructed to build a nonempty, convex, closed, and bounded subset $U=\left[\phi_{-}, \phi_{+}\right]_{\tilde{s}, p} \cap \bar{B}_{M} \subset W^{\tilde{s}, p}$, which is a strictly positive interval. These include variations of known barrier constructions which require the near-CMC condition, and also some new barrier constructions which are free of the near-CMC condition (Sect. 5). Note: This is the only place in the argument where near-CMC conditions may potentially arise.

Step 5. Application of fixed-point theorem. The global barriers and continuity properties are used together with the abstract topological fixed-point result (Theorem 5) to establish existence of solutions $\phi \in U \cap W^{s, p}$ and $w \in \boldsymbol{W}^{e, q}$ to the coupled system: $w=S(\phi), \phi=T(\phi, w)($ Sect. 6). 
Step 6. Bootstrap. The above application of a fixed-point theorem is actually performed for some low regularity spaces, i.e., for $s \leqslant 2$ and $e \leqslant 2$, and a bootstrap argument is then given to extend the results to the range of $s$ and $p$ given in the statement of the theorem (Sect. 6).

The ordered Banach space $Z$ plays a central role in Theorem 5. We will use $Z=W^{t, q}$, $t \geqslant 0,1 \leqslant q \leqslant \infty$, with order cone defined as in (2.5). Given such an order cone, one can define the closed interval

$$
\left[\phi_{-}, \phi_{+}\right]_{t, q}=\left\{\phi \in W^{t, q}: \phi_{-} \leqslant \phi \leqslant \phi_{+}\right\} \subset W^{t, q},
$$

which as noted earlier is denoted more simply as $\left[\phi_{-}, \phi_{+}\right]_{q}$ when $t=0$, and as simply $\left[\phi_{-}, \phi_{+}\right]$when $t=0, q=\infty$. When $t=0$, the $W^{t, q}$ order cone is normal for $1 \leqslant q \leqslant \infty$, meaning that closed intervals $\left[\phi_{-}, \phi_{+}\right]_{q} \subset L^{q}=W^{0, q}$ are automatically bounded in the metric given by the norm on $L^{q}$.

If we consider the interval $U=\left[\phi_{-}, \phi_{+}\right]_{t, q} \subset W^{t, q}=Z$ defined using this order structure, it will be critically important to establish that $U$ is convex (with respect to the vector space structure of $Z$ ), closed (in the topology of $Z$ ), and (when possible) bounded (in the metric given by the norm on $Z$ ). It will also be important that $U$ be nonempty as a subset of $Z$; this will involve choosing compatible $\phi_{-}$and $\phi_{+}$. Regarding convexity, closure, and boundedness, we have the following lemma.

Lemma 1 (Order cone intervals in $W^{t, q}$ ). For $t \geqslant 0,1 \leqslant q \leqslant \infty$, the set

$$
U=\left[\phi_{-}, \phi_{+}\right]_{t, q}=\left\{\phi \in W^{t, q}: \phi_{-} \leqslant \phi \leqslant \phi_{+}\right\} \subset W^{t, q}
$$

is convex with respect to the vector space structure of $W^{t, q}$ and closed in the topology of $W^{t, q}$. For $t=0,1 \leqslant q \leqslant \infty$, the set $U$ is also bounded with respect to the metric space structure of $L^{q}=W^{0, q}$.

Proof. That $U$ is convex for $t \geqslant 0,1 \leqslant q \leqslant \infty$, follows from the fact that any interval built using order cones is convex. That $U$ is closed in the case of $t=0,1 \leqslant q \leqslant \infty$ follows from the fact that norm convergence in $L^{q}$ for $1 \leqslant q \leqslant \infty$ implies pointwise subsequential convergence almost everywhere (see Theorem 3.12 in [44]). That $U$ is bounded when $t=0,1 \leqslant q \leqslant \infty$ follows from the fact that the order cone $L_{+}^{q}$ is normal (see Appendix A.2).

What remains is to show that $U$ is closed in the case of $t>0,1 \leqslant q \leqslant \infty$. The argument is as follows. Let $\left\{u_{k}\right\}_{k=1}^{\infty}$ be a Cauchy sequence in $U \subset W^{t, q} \subset L^{q}$, with $t>0,1 \leqslant q \leqslant \infty$. From completeness of $W^{t, q}$ there exists $\lim _{k \rightarrow \infty} u_{k}=u \in W^{t, q}$. From the continuous embedding $W^{t, q} \hookrightarrow L^{q}$ for $t>0$, we have that

$$
\left\|u_{k}-u_{l}\right\|_{q} \leqslant C\left\|u_{k}-u_{l}\right\|_{t, q}
$$

so that $u_{k}$ is also Cauchy in $L^{q}$. Moreover, the continuous embedding also implies that $u$ is also the limit of $u_{k}$ as a sequence in $L^{q}$. Since $\left[\phi_{-}, \phi_{+}\right]_{0, q}$ is closed in $L^{q}$, we have $u \in\left[\phi_{-}, \phi_{+}\right]_{0, q}$, and so $u \in U=\left[\phi_{-}, \phi_{+}\right]_{t, q}=\left[\phi_{-}, \phi_{+}\right]_{0, q} \cap W^{t, q}$.

Remark 2. We indicate now how the far-CMC result outlined in [22] can be recovered using Theorem 4 above. The framework is constructed by taking $X=W^{2, p}, Y=W^{2, p}$, and $Z=L^{\infty}$, with $p>3$, giving the compact embedding $W^{2, p} \hookrightarrow L^{\infty}$. The coefficients are assumed to satisfy $\tau \in W^{1, p}$ and $\sigma^{2}, j^{a}, \rho \in L^{p}$ as well as the assumptions for the construction of a near-CMC-free global super-solution (presented in [22] as Theorem 1, analogous to Lemma 9 in this paper), and for the construction of a near-CMC-free global 
sub-solution (presented in [22] as Theorem 2, analogous to Lemma 13 in this paper). One then takes $U=\left[\phi_{-}, \phi_{+}\right] \subset Z=L^{\infty}$, where the compatible $0<\phi_{-} \leqslant \phi_{+}$are these near-CMC-free barriers. Since $Z=L^{\infty}$ is an ordered Banach space with normal order cone, we have (by Lemma 1 in this paper) that $U$ is non-empty, convex, closed and bounded as a subset of $Z$. The invariance of the Picard mapping on the interval $\left[\phi_{-}, \phi_{+}\right]$ is proven using a monotone shift (cf. Lemma 4 in this paper) and a barrier argument (cf. Lemma 5 in this paper). The main result in [22] (stated in [22] as Theorem 4), now follows from Theorem 4 in this paper (stated in [22] as Lemma 1).

\section{Weak Solution Results for the Individual Constraints}

4.1. The momentum constraint and the solution map $S$. In this section we fix a particular scalar function $\phi \in W^{s, p}$ with $s p>3$, and consider separately the momentum constraint equation (2.24) to be solved for the vector valued function $\boldsymbol{w}$. The result is a linear elliptic system of equations for this variable $\boldsymbol{w}=\boldsymbol{w}_{\phi}$. For convenience, we reformulate the problem here in a self-contained manner. Note that the problem (4.2) below is identical to (2.24) provided the functions $\boldsymbol{b}_{\tau}$ and $\boldsymbol{b}_{j}$ are defined accordingly. Our goal is not only to develop some existence results for the momentum constraint, but also to derive the estimates for the momentum constraint solution map $S$ that we will need later in our analysis of the coupled system. We note that a complete weak solution theory for the momentum constraint on compact manifolds with boundary, using both variational methods and Riesz-Schauder Theory, is developed in [21].

Let $(\mathcal{M}, h)$ be a 3-dimensional Riemannian manifold, where $\mathcal{M}$ is a smooth, compact manifold without boundary, and with $p \in(1, \infty)$ and $s \in\left(\frac{3}{p}, \infty\right), h \in W^{s, p}$ is a positive definite metric. With

$$
q \in(1, \infty), \quad \text { and } \quad e \in(2-s, s] \cap\left(-s+\frac{3}{p}-1+\frac{3}{q}, s-\frac{3}{p}+\frac{3}{q}\right],
$$

introduce the bounded linear operator

$$
A_{\mathbb{L}}: \boldsymbol{W}^{e, q} \rightarrow \boldsymbol{W}^{e-2, q},
$$

as the unique extension of the operator $\mathbb{L}$ in (2.17), cf. Lemmata 31 and 32 in Appendix A.5. Fix the source terms $\boldsymbol{b}_{\tau}, \boldsymbol{b}_{j} \in \boldsymbol{W}^{e-2, q}$. Fix a function $\phi \in W^{s, p}$, and define

$$
\boldsymbol{f}_{\phi} \in \boldsymbol{W}^{s-2, q}, \quad \boldsymbol{f}_{\phi}:=\boldsymbol{b}_{\tau} \phi^{6}+\boldsymbol{b}_{j}
$$

We used the subscript $\phi$ in $f_{\phi}$ to emphasize that $\phi$ is not a variable (but the "source") of the problem. Note that the above conditions on $q$ and $e$ are sufficient for the pointwise multiplication by an element of $W^{s, p}$ to be a bounded map in $\boldsymbol{W}^{e-2, q}$, cf. Corollary 3(a) in Appendix A.4.

The momentum constraint equation is the following: find an element $\boldsymbol{w} \in \boldsymbol{W}^{e, q}$ solution of

$$
A_{\mathbb{L}} \boldsymbol{w}+\boldsymbol{f}_{\phi}=0 .
$$

We sketch here a proof of existence of weak solutions of the momentum constraint equation (4.2). 
Theorem 6 (Momentum constraint). Let e and $q$ be as above. Then there exists a solution $\boldsymbol{w} \in \boldsymbol{W}^{e, q}$ to the momentum constraint equation (4.2) if and only if $\boldsymbol{f}_{\phi}(\boldsymbol{v})=0$ for all $\boldsymbol{v} \in \boldsymbol{W}^{2-e, q^{\prime}}$ satisfying $A_{\mathbb{L}}^{*} \boldsymbol{v}=0$. The solution is unique if and only if the kernel of $A_{\mathbb{L}}^{*}$ is trivial. Moreover, if a solution exists at all in $\boldsymbol{W}^{e, q}$, for any given closed linear space $K \subseteq \boldsymbol{W}^{e, q}$ such that $\boldsymbol{W}^{e, q}=\operatorname{ker} A_{\mathbb{L}} \oplus K$, there is a unique solution satisfying $\boldsymbol{w} \in K$, and for this solution, we have

$$
\|\boldsymbol{w}\|_{e, q} \leqslant C\left\|\boldsymbol{f}_{\phi}\right\|_{e-2, q}
$$

with some constant $C>0$ not depending on $\boldsymbol{w}$.

Proof. By Lemma 34 in Appendix A.5, the operator $A_{\mathbb{L}}$ is semi-Fredholm, and moreover since $A_{\mathbb{L}}$ is formally self-adjoint, it is Fredholm. The formal self-adjointness also implies that when the metric is smooth, index of $A_{\mathbb{L}}$ is zero independent of $e$ and $q$. Now we can approximate the metric $h$ by smooth metrics so that $A_{\mathbb{L}}$ is sufficiently close to a Fredholm operator with index zero. Since the set of Fredholm operators with constant index is open, we conclude that the index of $A_{\mathbb{L}}$ is zero, and the theorem follows.

In the later sections we need to bound the coefficient $a_{w}$ in the Hamiltonian constraint equation, which can be obtained by using the following observation.

Corollary 1. Let $p \in(1, \infty)$ and $s \in\left(1+\frac{3}{p}, \infty\right)$. In addition, let $q \in(3, \infty)$ and $e \in(1, s] \cap\left(1+\frac{3}{q}, s-\frac{3}{p}+\frac{3}{q}\right] \cap(1,2]$, and with $z=\frac{3 q}{3+(2-e) q}$, let $\boldsymbol{b}_{\tau} \in \boldsymbol{L}^{z}$. Assume that the momentum constraint equation has a solution $\boldsymbol{w} \in \boldsymbol{W}^{e, q}$. Then, we have

$$
\|\mathcal{L} \boldsymbol{w}\|_{\infty} \leqslant C\|\phi\|_{\infty}^{6}\left\|\boldsymbol{b}_{\tau}\right\|_{z}+C\left\|\boldsymbol{b}_{j}\right\|_{e-2, q},
$$

with $C>0$ not depending on $\boldsymbol{w}$. Moreover, if the solution is unique, the norm $\|\boldsymbol{w}\|_{e, q}$ can be bounded by the same expression.

Proof. Since the kernel of $A_{\mathbb{L}}$ is finite dimensional, we can write $\boldsymbol{W}^{e, q}=\operatorname{ker} A_{\mathbb{L}} \oplus K$ with a closed linear space $K \subseteq \boldsymbol{W}^{e, q}$. We have the splitting $\boldsymbol{w}=\boldsymbol{w}_{0}+\boldsymbol{w}_{1}$ such that $\boldsymbol{w}_{0} \in \operatorname{ker} A_{\mathbb{L}}=\operatorname{ker} \mathcal{L}$ and $\boldsymbol{w}_{1} \in K$, implying that

$$
\|\mathcal{L} \boldsymbol{w}\|_{\infty}=\left\|\mathcal{L} \boldsymbol{w}_{1}\right\|_{\infty} \leqslant c\left\|\boldsymbol{w}_{1}\right\|_{1, \infty} \leqslant c^{\prime}\left\|\boldsymbol{w}_{1}\right\|_{e, q},
$$

the latter inequality by $\boldsymbol{W}^{e, q} \hookrightarrow \boldsymbol{W}^{1, \infty}$. We note that demanding $\boldsymbol{W}^{e, q} \hookrightarrow \boldsymbol{W}^{1, \infty}$ gives us the lower bound $e>1+\frac{3}{q}$, and this in turn implies $s>1+\frac{3}{p}$ if the range of $e$ is to be nonempty. To complete the proof, we note that $\boldsymbol{w}_{1}$ is also a solution of the momentum constraint, and taking into account $\boldsymbol{L}^{z} \hookrightarrow \boldsymbol{W}^{e-2, q}$, we apply Theorem 6 to bound the norm $\left\|\boldsymbol{w}_{1}\right\|_{e, q}$. Note that the latter embedding requires $e \leqslant 2$, and combining this with $e>1+\frac{3}{q}$, we need $q>3$.

We now establish some properties of the momentum constraint solution map $S$ that we will need later for our analysis of the coupled system. Suppose that the conditions for Theorem 6 hold, so that the momentum constraint is uniquely solvable. Then for any fixed $\phi_{+} \in W^{s, p}$ with $\phi_{+}>0$, there exists a mapping

$$
S:\left[0, \phi_{+}\right] \cap W^{s, p} \rightarrow \boldsymbol{W}^{e, q}
$$

that sends the source $\phi$ to the corresponding solution $w$ of the momentum constraint equation. Since the momentum constraint is linear, it follows easily that $S$ is Lipschitz continuous as stated in the following lemma. 
Lemma 2 (Properties of the map $S$ ). In addition to the conditions imposed in the beginning of this section, let $s \geqslant 1$. Let $e \in[1,3]$ and $\frac{1}{q} \in\left(\frac{e-1}{2} \delta, 1-\frac{3-e}{2} \delta\right)$, where $\delta=\max \left\{0, \frac{1}{p}-\frac{s-1}{3}\right\}$. Assume that the momentum constraint (4.2) is uniquely solvable in $\boldsymbol{W}^{e, q}$. With some $\phi_{+} \in W^{s, p}$ satisfying $\phi_{+}>0$, let $\boldsymbol{w}_{1}$ and $\boldsymbol{w}_{2}$ be the solutions to the momentum constraint with the source functions $\phi_{1}$ and $\phi_{2}$ from the set $\left[0, \phi_{+}\right] \cap W^{s, p}$, respectively. Then,

$$
\left\|\boldsymbol{w}_{1}-\boldsymbol{w}_{2}\right\|_{e, q} \leqslant C\left\|\phi_{+}\right\|_{\infty}^{5}\left\|\boldsymbol{b}_{\tau}\right\|_{e-2, q}\left\|\phi_{1}-\phi_{2}\right\|_{s, p} .
$$

Proof. The functions $\phi_{1}$ and $\phi_{2}$ pointwise satisfy the following inequalities:

$$
\begin{aligned}
\phi_{2}^{n}-\phi_{1}^{n} & =\left(\sum_{j=0}^{n-1} \phi_{2}^{j} \phi_{1}^{n-1-j}\right)\left(\phi_{2}-\phi_{1}\right) \leqslant n\left(\phi_{+}\right)^{n-1}\left|\phi_{2}-\phi_{1}\right|, \\
-\left[\phi_{2}^{-n}-\phi_{1}^{-n}\right] & =\frac{\phi_{2}^{n}-\phi_{1}^{n}}{\left(\phi_{2} \phi_{1}\right)^{n}} \leqslant n \frac{\left(\phi_{+}\right)^{n-1}}{\left(\phi_{-}\right)^{2 n}}\left|\phi_{2}-\phi_{1}\right|,
\end{aligned}
$$

for any integer $n>0$. Since Eq. (4.2) is linear, applying Theorem 6 with the right-hand side $\boldsymbol{f}:=\boldsymbol{f}_{\phi_{1}}-\boldsymbol{f}_{\phi_{2}}$, and by using Lemma 29 in Appendix, we obtain

$$
\left\|\boldsymbol{w}_{1}-\boldsymbol{w}_{2}\right\|_{e, q} \leqslant\left\|\boldsymbol{b}_{\tau}\right\|_{e-2, q}\left\|\phi_{1}^{6}-\phi_{2}^{6}\right\|_{s, p} \leqslant 6\left\|\phi_{+}\right\|_{\infty}^{5}\left\|\boldsymbol{b}_{\tau}\right\|_{e-2, q}\left\|\phi_{1}-\phi_{2}\right\|_{s, p} .
$$

4.2. The Hamiltonian constraint and the Picard map T. In this section we fix a particular function $a_{w}$ in an appropriate space and we then separately look for weak solutions of the Hamiltonian constraint equation (2.23). For convenience, we reformulate the problem here in a self-contained manner. Note that the problem (4.9) below is identical to (2.23), provided the functionals $a_{\tau}$ and $a_{\rho}$ are defined accordingly. Our goal here is primarily to establish some properties and derive some estimates for a Hamiltonian constraint fixed-point map $T$ that we will need later in our analysis of the coupled system, and also for the analysis of the Hamiltonian constraint alone in the CMC setting. We remark that a complete weak solution theory for the Hamiltonian constraint on compact manifolds with boundary, using both variational methods and fixed-point arguments based on monotone increasing maps, combined with sub- and super-solutions, is developed in [21].

Let $(\mathcal{M}, h)$ be a 3-dimensional Riemannian manifold, where $\mathcal{M}$ is a smooth, compact manifold without boundary, and with $p \in(1, \infty)$ and $s \in\left(\frac{3}{p}, \infty\right) \cap[1, \infty), h \in W^{s, p}$ is a positive definite metric. Introduce the operator

$$
A_{L}: W^{s, p} \rightarrow W^{s-2, p}
$$

as the unique extension of the Laplace-Beltrami operator $L=-\Delta$, cf. Lemma 31 in Appendix A.5. Fix the source functions

$$
a_{\tau}, a_{\rho}, a_{w} \in W_{+}^{s-2, p}, \quad \text { and } \quad a_{R}=\frac{1}{8} R \in W^{s-2, p},
$$

where $R$ is the scalar curvature of the metric $h$. (By Corollary 3(b) in Appendix A.4, we know $h_{a b} \in W^{s, p}$ implies $R \in W^{s-2, p}$.) Given any two functions $\phi_{-}, \phi_{+} \in W^{s, p}$ with $0<\phi_{-} \leqslant \phi_{+}$, introduce the nonlinear operator

$$
f_{w}:\left[\phi_{-}, \phi_{+}\right]_{s, p} \rightarrow W^{s-2, p}, \quad f_{w}(\phi)=a_{\tau} \phi^{5}+a_{R} \phi-a_{\rho} \phi^{-3}-a_{w} \phi^{-7},
$$


where the pointwise multiplication by an element of $W^{s, p}$ defines a bounded linear map in $W^{s-2, p}$ since $s-2 \geqslant-s$ and $2\left(s-\frac{3}{p}\right)>0>2-3$, cf. Corollary 3(a) in Appendix A.4. In case the coupled system is under consideration, the dependence of $f_{w}$ on $w$ is hidden in the fact that the coefficient $a_{w}$ depends on $w$, cf. (2.18). For generality, in the following we will view that the operator $f_{w}$ depends on $a_{w}$.

We now formulate the Hamiltonian constraint equation as follows: find an element $\phi \in\left[\phi_{-}, \phi_{+}\right]_{s, p}$ solution of

$$
A_{L} \phi+f_{w}(\phi)=0 .
$$

To establish existence results for weak solutions to the Hamiltonian constraint equation using fixed-point arguments, we will rely on the existence of generalized (weak) suband super-solutions (sometimes called barriers) which will be derived later in Sect. 5. Let us recall the definition of sub- and super-solutions in the following, in a slightly generalized form that will be necessary in our study of the coupled system.

A function $\phi_{-} \in(0, \infty) \cap W^{s, p}$ is called a sub-solution of (2.23) iff the function $\phi_{-}$satisfies the inequality

$$
A_{L} \phi_{-}+f_{w}\left(\phi_{-}\right) \leqslant 0,
$$

for some $a_{w} \in W^{s-2, p}$. A function $\phi_{+} \in(0, \infty) \cap W^{s, p}$ is called a super-solution of (2.23) iff the function $\phi_{+}$satisfies the inequality

$$
A_{L} \phi_{+}+f_{w}\left(\phi_{+}\right) \geqslant 0
$$

for some $a_{w} \in W^{s-2, p}$. We say a pair of sub- and super-solutions is compatible if they satisfy

$$
0<\phi_{-} \leqslant \phi_{+}<\infty
$$

so that the interval $\left[\phi_{-}, \phi_{+}\right] \cap W^{s, p}$ is both nonempty and bounded.

We now turn to the construction of the fixed-point mapping $T: U \times \mathcal{R}(S) \rightarrow X$ for the Hamiltonian constraint and its properties. There are a number of possibilities for defining $T$; the requirements are (1) that every fixed-point of $T$ must be a solution to the Hamiltonian constraint; (2) $T$ must be a continuous map from its domain to its range; and (3) $T$ must be invariant on a non-empty, convex, closed, bounded subset $U$ of an ordered Banach space $Z$, with $X \hookrightarrow Z$ compact. It will be sufficient to define $T$ using a variation of the Picard iteration as follows. Due to the presence of the non-trivial kernel of the operator $A_{L}$, which is a consequence of working with a closed manifold, we must introduce a shift into the Hamiltonian constraint equation in order to construct $T$ with the required properties.

Lemma 3 (Properties of the map $T$ ). In the above described setting, assume that $p \in$ $\left(\frac{3}{2}, \infty\right)$ and $s \in\left(\frac{3}{p}, \infty\right) \cap[1,3]$. With $a_{0} \in W_{+}^{s-2, p}$ satisfying $a_{0} \neq 0$, and $\psi \in W_{+}^{s, p}$, let $a_{s}=a_{0}+a_{w} \psi \in W^{s-2, p}$. Fix the functions $\phi_{-}, \phi_{+} \in W^{s, p}$ such that $0<\phi_{-} \leqslant \phi_{+}$, and define the shifted operators

$$
\begin{aligned}
A_{L}^{s}: W^{s, p} \rightarrow W^{s-2, p}, & A_{L}^{s} \phi & :=A_{L} \phi+a_{s} \phi, \\
f_{w}^{s}:\left[\phi_{-}, \phi_{+}\right]_{s, p} \rightarrow W^{s-2, p}, & f_{w}^{s}(\phi) & :=f_{w}(\phi)-a_{s} \phi .
\end{aligned}
$$

Let, for $\phi \in\left[\phi_{-}, \phi_{+}\right]_{s, p}$ and $a_{w} \in W^{s-2, p}$,

$$
T^{s}\left(\phi, a_{w}\right):=-\left(A_{L}^{s}\right)^{-1} f_{w}^{s}(\phi) .
$$


Then, the map $T^{s}:\left[\phi_{-}, \phi_{+}\right]_{s, p} \times W^{s-2, p} \rightarrow W^{s, p}$ is continuous in both arguments. Moreover, there exist $\tilde{s} \in\left(\frac{3}{p}, s\right)$ and a constant $C$ such that

$$
\left\|T\left(\phi, a_{w}\right)\right\|_{s, p} \leqslant C\left(1+\left\|a_{w}\right\|_{s-2, p}\right)\|\phi\|_{\tilde{s}, p},
$$

for all $\phi \in\left[\phi_{-}, \phi_{+}\right]_{s, p}$ and $a_{w} \in W^{s-2, p}$.

Proof. In this proof, we denote by $C$ a generic constant that may have different values at its different occurrences. By applying Lemma 29 from the Appendix, for any $\tilde{s} \in\left(\frac{3}{p}, s\right]$, $s-2 \in[-1,1]$ and $\frac{1}{p} \in\left(\frac{s-1}{2} \delta, 1-\frac{3-s}{2} \delta\right)$ with $\delta=\frac{1}{p}-\frac{\tilde{s}-1}{3}$, we have

$$
\begin{aligned}
\left\|f_{w}^{s}(\phi)\right\|_{s-2, p} \leqslant & C\left(\left\|a_{\tau}\right\|_{s-2, p}\left\|\phi_{+}^{4}\right\|_{\infty}+\left\|a_{\rho}\right\|_{s-2, p}\left\|\phi_{-}^{-4}\right\|_{\infty}\right. \\
& \left.+\left\|a_{w}\right\|_{s-2, p}\left(\left\|\phi_{-}^{-8}\right\|_{\infty}+\|\psi\|_{\tilde{s}, p}\right)+\left\|a_{R}+a_{0}\right\|_{s-2, p}\right)\|\phi\|_{\tilde{s}, p} .
\end{aligned}
$$

Let us verify if $\frac{1}{p}$ is indeed in the prescribed range. First, we have $\delta=\frac{1}{3}+\frac{1}{p}-\frac{\tilde{s}}{3}<\frac{1}{3}$ since $\frac{\tilde{s}}{3}-\frac{1}{p}>0$, and taking into account $s \geqslant 1$, we infer $1-\frac{3-s}{2} \delta \geqslant 1-\frac{3-1}{2} \frac{1}{3}=\frac{2}{3}$. This shows $\frac{1}{p}<1-\frac{3-s}{2} \delta$ for $p>\frac{3}{2}$, which is not sharp, but will be sufficient for our analysis. For the other bound, we need $\frac{1}{p}<\frac{s-1}{2} \delta=\frac{s-1}{2 p}-\frac{(s-1)(\tilde{s}-1)}{6}$, or in other words, $\frac{(s-1)(\tilde{s}-1)}{6}>\frac{s-3}{2 p}$. Since $s \in[1,3]$, it is possible to choose $\tilde{s} \in\left(\frac{3}{p}, s\right]$ satisfying this inequality.

To finalize the proof of (4.16), we note that by Lemma 36 in Appendix A.6, the operator $A_{L}^{s}$ is invertible, since the function $a_{s}$ is positive, and that by Corollary 5 also in that appendix, the inverse $\left(A_{L}^{s}\right)^{-1}: W^{s-2, p} \rightarrow W^{s, p}$ is bounded.

The continuity of the mapping $f_{w}^{s}:\left[\phi_{-}, \phi_{+}\right]_{s, p} \rightarrow W^{s-2, p}$ for any $a_{w} \in W^{s-2, p}$ is obtained similarly, and the continuity of $a_{w} \mapsto f_{w}(\phi)$ for fixed $\phi \in\left[\phi_{-}, \phi_{+}\right]_{s, p}$ is obvious. Being the composition of continuous maps, $\left(\phi, a_{w}\right) \mapsto T_{w}^{s}(\phi)$ is also continuous.

The following lemma shows that by choosing the shift sufficiently large, we can make the map $T^{s}$ monotone increasing. This result is important for ensuring that the Picard map $T$ for the Hamiltonian constraint is invariant on the interval $\left[\phi_{-}, \phi_{+}\right]$defined by sub- and super-solutions. There is an obstruction that the scalar curvature should be continuous, which will be handled in the general case by conformally transforming the metric to a metric with continuous scalar curvature and using the conformal covariance of the Hamiltonian constraint, cf. Sect. 6.1.

Lemma 4 (Monotone increasing property of $T$ ). In addition to the conditions of Lemma 3 , let $a_{R}$ be continuous and define the shift function $a_{s}$ by

$$
a_{s}=\max \left\{1, a_{R}\right\}+3 \frac{\phi_{+}^{2}}{\phi_{-}^{6}} a_{\rho}+5 \phi_{+}^{4} a_{\tau}+7 \frac{\phi_{+}^{6}}{\phi_{-}^{14}} a_{w} .
$$

Then, for any fixed $a_{w} \in W^{s-2, p}$, the map $\phi \mapsto T^{s}\left(\phi, a_{w}\right):\left[\phi_{-}, \phi_{+}\right]_{s, p} \rightarrow W^{s, p}$ is monotone increasing. 
Proof. The shifted operator $A_{L}^{s}$ satisfies the maximum principle, hence the inverse $\left(A_{L}^{s}\right)^{-1}: W^{s-2, p} \rightarrow W^{s, p}$ is monotone increasing.

Now we will show that the operator $f_{w}^{s}$ is monotone decreasing. Given any two functions $\phi_{2}, \phi_{1} \in\left[\phi_{-}, \phi_{+}\right]_{s, p}$ with $\phi_{2} \geqslant \phi_{1}$, we have

$$
\begin{aligned}
f_{w}^{s}\left(\phi_{2}\right)-f_{w}^{s}\left(\phi_{1}\right)= & f_{w}\left(\phi_{2}\right)-f_{w}\left(\phi_{1}\right)-a_{s}\left[\phi_{2}-\phi_{1}\right] \\
= & a_{\tau}\left[\phi_{2}^{5}-\phi_{1}^{5}\right]+a_{R}\left[\phi_{2}-\phi_{1}\right]-a_{s}\left[\phi_{2}-\phi_{1}\right]-a_{\rho}\left[\phi_{2}^{-3}-\phi_{1}^{-3}\right] \\
& -a_{w}\left[\phi_{2}^{-7}-\phi_{1}^{-7}\right] .
\end{aligned}
$$

The inequalities (4.7), the condition $0<\phi_{1} \leqslant \phi_{2}$, and the choice of $a_{s}$ imply

$$
f_{w}^{s}\left(\phi_{2}\right)-f_{w}^{s}\left(\phi_{1}\right) \leqslant 0
$$

which establishes that $f_{w}^{s}$ is monotone decreasing.

Both the operator $\left(A_{L}^{s}\right)^{-1}$ and the map $-f_{w}^{s}$ are monotone increasing, therefore the operator $T^{s}\left(\cdot, a_{w}\right)$ is also monotone increasing.

Lemma 5 (Barriers for $T$ and the Hamiltonian constraint). Let the conditions of Lemma 4 hold, with $\phi_{-}$and $\phi_{+}$sub-and super-solutions of the Hamiltonian constraint equation (4.9), respectively. Then, we have $T^{s}\left(\phi_{+}, a_{w}\right) \leqslant \phi_{+}$and $T^{s}\left(\phi_{-}, a_{w}\right) \geqslant \phi_{-}$.

Proof. We have

$$
\phi_{+}-T^{s}\left(\phi_{+}, a_{w}\right)=\left(A_{L}^{s}\right)^{-1}\left[A_{L}^{s} \phi_{+}+f_{w}^{s}\left(\phi_{+}\right)\right]
$$

which is nonnegative since $\phi_{+}$is a super-solution and $\left(A_{L}^{S}\right)^{-1}$ is linear and monotone increasing. The proof of the other inequality is completely analogous.

Since we are no longer using normal order cones, our non-empty, convex, closed interval $\left[\phi_{-}, \phi_{+}\right]_{s, p}$ is not necessarily bounded as a subset of $W^{s, p}$. Therefore, we also need $a$ priori bounds in the norm on $W^{s, p}$ to ensure the Picard iterates stay inside the intersection of the interval with the closed ball $\bar{B}_{M}$ in $W^{s, p}$ of radius $M$, centered at the origin. We first establish a lemma to this effect that will be useful for both the non-CMC and CMC cases.

Lemma 6 (Invariance of $T$ on the ball $\bar{B}_{M}$ ). Let the conditions of Lemma 3 hold, and let $a_{w} \in W^{s-2, p}$. Then, for any $\tilde{s} \in\left(\frac{3}{p}, s\right]$ and for some $t \in\left(\frac{3}{p}, \tilde{s}\right)$ there exists a closed ball $\bar{B}_{M} \subset W^{\tilde{s}, p}$ of radius $M=\mathcal{O}\left(\left[1+\left\|a_{w}\right\|_{s-2, p}\right]^{\tilde{s} /(\tilde{s}-t)}\right)$, such that

$$
\phi \in\left[\phi_{-}, \phi_{+}\right]_{\tilde{s}, p} \cap \bar{B}_{M} \Rightarrow T^{s}\left(\phi, a_{w}\right) \in \bar{B}_{M} .
$$

Proof. From Lemma 3, there exist $t \in\left(\frac{3}{p}, \tilde{s}\right)$ and $K>0$ such that

$$
\left\|T^{s}\left(\phi, a_{w}\right)\right\|_{\tilde{s}, p} \leqslant K\left(1+\left\|a_{w}\right\|_{s-2, p}\right)\|\phi\|_{t, p}, \quad \forall \phi \in\left[\phi_{-}, \phi_{+}\right]_{\tilde{s}, p} .
$$

For any $\varepsilon>0$, the norm $\|\phi\|_{t, p}$ can be bounded by the interpolation estimate

$$
\|\phi\|_{t, p} \leqslant \varepsilon\|\phi\|_{\tilde{s}, p}+C \varepsilon^{-t /(\tilde{s}-t)}\|\phi\|_{p}
$$


where $C$ is a constant independent of $\varepsilon$. Since $\phi$ is bounded from above by $\phi_{+},\|\phi\|_{p}$ is bounded uniformly, and now demanding that $\phi \in \bar{B}_{M}$, we get

$$
\left\|T^{s}\left(\phi, a_{w}\right)\right\|_{\tilde{s}, p} \leqslant K\left[1+\left\|a_{w}\right\|_{s-2, p}\right]\left(M \varepsilon+C \varepsilon^{-t /(\tilde{s}-t)}\right),
$$

with possibly different constant $C$. Choosing $\varepsilon$ such that $2 \varepsilon K\left[1+\left\|a_{w}\right\|_{s-2, p}\right]=1$ and setting $M=2 K C\left[1+\left\|a_{w}\right\|_{s-2, p}\right] \varepsilon^{-t /(\tilde{s}-t)}$, we can ensure that the right-hand side of (4.17) is bounded by $M$.

\section{Barriers for the Hamiltonian Constraint}

The results developed in Sect. 4.2 for a particular fixed-point map $T$ for analyzing the Hamiltonian constraint equation and the coupled system rely on the existence of generalized (weak) sub- and super-solutions, or barriers. There, the Hamiltonian constraint was studied in isolation from the momentum constraint, and these generalized barriers only needed to satisfy the conditions given at the beginning of Sect. 4.2 for a given fixed function $\boldsymbol{w}$ appearing as a source term in the nonlinearity of the Hamiltonian constraint. Therefore, these types of barriers are sometimes referred to as local barriers, in that the coupling to the momentum constraint is ignored. In order to establish existence results for the coupled system in the non-CMC case, it will be critical that the sub- and super-solutions satisfy one additional property that now reflects the coupling, giving rise to the term global barriers. It will be useful now to define this global property precisely.

Definition 1. A sub-solution $\phi_{-}$is called global iff it is a sub-solution of (2.23) for all vector fields $\boldsymbol{w}_{\phi}$ solution of (2.24) with source function $\phi \in\left[\phi_{-}, \infty\right) \cap W^{s, p}$. A supersolution $\phi_{+}$is called global iff it is a super-solution of (2.23) for all vector fields $\boldsymbol{w}_{\phi}$ solution of (2.24) with source function $\phi \in\left(0, \phi_{+}\right] \cap W^{s, p}$. A pair $\phi_{-} \leqslant \phi_{+}$of sub-and super-solutions is called an admissible pair if $\phi_{-}$and $\phi_{+}$are sub-and super-solutions of (2.23) for all vector fields $\boldsymbol{w}_{\phi}$ of (2.24) with source function $\phi \in\left[\phi_{-}, \phi_{+}\right] \cap W^{s, p}$.

It is obvious that if $\phi_{-}$and $\phi_{+}$are respectively global sub- and super-solutions, then the pair $\phi_{-}, \phi_{+}$is admissible in the sense above, provided they satisfy the compatibility condition (4.12).

Below we give a number of (local and global) sub- and super-solution constructions for closed manifolds; analogous constructions for compact manifolds with boundary are given in [21]. These constructions are based on generalizing known constant sub- and super-solution constructions given previously in the literature for closed manifolds. On one hand, the generalized global sub-solution constructions appearing here and in [21] do not require the near-CMC condition, inheriting this property from the known sub-solutions from literature on which they are based. However, on the other hand, all previously known global super-solutions for the Hamiltonian constraint equation have required the near-CMC condition.

Here and in [21,22], one of our primary interests is in developing existence results for weak (and strong) non-CMC solutions to the coupled system which are free of the near-CMC assumption. This assumption had appeared in two distinct places in all prior literature on this problem [1,26]; the first assumption appears in the construction of a fixed-point argument based on strict $k$-contractions, and the second assumption appears in the construction of global super-solutions. Here and in [21,22], an alternative fixedpoint framework based on compactness arguments rather than $k$-contractions is used 
to remove the first of these near-CMC assumptions. In this section, we give some new constructions of global super-solutions that are free of the near-CMC assumption, along with some compatible sub-solutions. These sub- and super-solution constructions are needed (without their global property) for the existence result for the Hamiltonian constraint (Theorem 3), and they are also needed (now with their global property) for the general fixed-point result for the coupled system (Theorem 5), leading to our two main non-CMC results (Theorems 1 and Theorem 2). The super-solutions in Lemmata 7(b) and 9 appear to be the first such near-CMC-free constructions, and provide the second key piece of the puzzle we need in order to establish non-CMC results through Theorem 5 without the near-CMC condition.

Throughout this section, we will assume that the background metric $h$ belongs to $W^{s, p}$ with $p \in(1, \infty)$ and $s \in\left(\frac{3}{p}, \infty\right) \cap(1,2]$. Recall that $r=\frac{3 p}{3+(2-s) p}$, so that the continuous embedding $L^{r} \hookrightarrow W^{s-2, p}$ holds. Given a symmetric two-index tensor $\sigma \in L^{2 r}$ and a vector field $\boldsymbol{w} \in \boldsymbol{W}^{1,2 r}$, introduce the functions $a_{\sigma}=\frac{1}{8} \sigma^{2} \in L^{r}$ and $a_{\mathcal{L} \boldsymbol{w}}=\frac{1}{8}(\mathcal{L} \boldsymbol{w})^{2} \in L^{r}$. Note that under these conditions $a_{\boldsymbol{w}}$ belongs to $L^{r} \hookrightarrow W^{s-2,2}$, and that if $a_{\sigma}, a_{\mathcal{L} w} \in L^{\infty}$ we have the pointwise estimate

$$
a_{\boldsymbol{w}}^{\wedge} \leqslant 2 a_{\sigma}^{\wedge}+2 a_{\mathcal{L} w}^{\wedge}
$$

Here and in what follows, given any scalar function $u \in L^{\infty}$, we use the notation

$$
u^{\wedge}:=\operatorname{ess} \sup u, \quad u^{\vee}:=\operatorname{ess} \inf u .
$$

In some places we will assume that when the vector field $\boldsymbol{w} \in \boldsymbol{W}^{1,2 r}$ is given by the solution of the momentum constraint equation (2.24) (or (4.2)) with the source term $\phi \in W^{s, p}$,

$$
a_{\mathcal{L} w}^{\wedge} \leqslant \mathrm{k}(\phi):=\mathrm{k}_{1}\|\phi\|_{\infty}^{12}+\mathrm{k}_{2}
$$

with some positive constants $\mathrm{k}_{1}$ and $\mathrm{k}_{2}$. We can verify this assumption e.g. when the conditions of Corollary 1 are satisfied, since from Corollary 1 we would get

$$
a_{\mathcal{L} w}^{\wedge}=\|\mathcal{L} w\|_{\infty}^{2} \leqslant C^{2}\left(\|\phi\|_{\infty}^{6}\left\|\boldsymbol{b}_{\tau}\right\|_{z}+\left\|\boldsymbol{b}_{j}\right\|_{e-2, q}\right)^{2},
$$

giving the bound (5.1) with the constants

$$
\mathrm{k}_{1}=2 C^{2}\left\|\boldsymbol{b}_{\tau}\right\|_{z}^{2}, \quad \text { and } \quad \mathrm{k}_{2}=2 C^{2}\left\|\boldsymbol{b}_{j}\right\|_{e-2, q}^{2} .
$$

5.1. Constant barriers. Now we will present some global sub- and super-solutions for the Hamiltonian constraint equation (2.23) which are constant functions. The proofs essentially follow the arguments in [21] for the case of compact manifolds with boundary.

Lemma 7 (Global super-solution). Let $(\mathcal{M}, h)$ be a 3-dimensional, smooth, closed Riemannian manifold with metric $h \in W^{s, p}$. Assume that the estimate (5.1) holds for the solution of the momentum constraint equation, and assume that $a_{\rho}, a_{\sigma} \in L^{\infty}$ and that $a_{R}$ is uniformly bounded from below. With the parameter $\varepsilon>0$ to be chosen later, define the rational polynomial

$$
q_{\varepsilon}(\chi)=\left(a_{\tau}^{\vee}-\mathrm{K}_{1 \varepsilon}\right) \chi^{5}+a_{R}^{\vee} \chi-a_{\rho}^{\wedge} \chi^{-3}-\mathrm{K}_{2 \varepsilon} \chi^{-7},
$$

where $\mathrm{K}_{1 \varepsilon}:=\left(1+\frac{1}{\varepsilon}\right) \mathrm{k}_{1}$ and $\mathrm{K}_{2 \varepsilon}:=(1+\varepsilon) a_{\sigma}^{\wedge}+\left(1+\frac{1}{\varepsilon}\right) \mathrm{k}_{2}$. We distinguish the following two cases: 
(a) In case $\mathrm{k}_{1}<a_{\tau}^{\vee}$, choose $\varepsilon>\frac{\mathrm{k}_{1}}{a_{\tau}^{\vee}-\mathrm{k}_{1}}$. If $q_{\varepsilon}$ has a root, let $\phi_{+}=\phi_{1}\left(a_{\tau}^{\vee}\right.$ $\left.\mathrm{K}_{1 \varepsilon}, a_{R}^{\vee}, a_{\rho}^{\wedge}, \mathrm{K}_{2 \varepsilon}\right)$ be the largest positive root of $q$, and if $q$ has no positive roots, let $\phi_{+}=1$. Now, the constant $\phi_{+}$is a global super-solution of the Hamiltonian constraint equation (2.23).

(b) In case $\mathrm{k}_{1} \geqslant a_{\tau}^{\vee}$, choose $\varepsilon>0$. In addition, assume that $a_{R}^{\vee}>0$ and that both $a_{\rho}^{\wedge}$ and $\mathrm{K}_{2 \varepsilon}$ are sufficiently small, such that $q$ has two positive roots. Then, the largest root $\phi_{+}=\phi_{2}\left(a_{\tau}^{\vee}-\mathrm{K}_{1 \varepsilon}, a_{R}^{\vee}, a_{\rho}^{\wedge}, \mathrm{K}_{2 \varepsilon}\right)$ of $q$ is a super-solution of the Hamiltonian constraint equation (2.23).

Proof. We look for a super-solution among the constant functions. Let $\chi$ be any positive constant. Then we have

$$
f(\chi, w)=a_{\tau} \chi^{5}+a_{R} \chi-a_{\rho} \chi^{-3}-a_{w} \chi^{-7} \geqslant a_{\tau}^{\vee} \chi^{5}+a_{R}^{\vee} \chi-a_{\rho}^{\wedge} \chi^{-3}-a_{w}^{\wedge} \chi^{-7} .
$$

Given any $\varepsilon>0$, the inequality $2\left|\sigma_{a b}(\mathcal{L} \boldsymbol{w})^{a b}\right| \leqslant \varepsilon \sigma^{2}+\frac{1}{\varepsilon}(\mathcal{L} \boldsymbol{w})^{2}$ implies that

$$
8 a_{w}=\sigma^{2}+(\mathcal{L} \boldsymbol{w})^{2}+2 \sigma_{a b}(\mathcal{L} \boldsymbol{w})^{a b} \leqslant(1+\varepsilon) \sigma^{2}+\left(1+\frac{1}{\varepsilon}\right)(\mathcal{L} \boldsymbol{w})^{2},
$$

hence, taking into account (5.1), for any $\boldsymbol{w} \in \boldsymbol{W}^{1,2 r}$ that is a solution of the momentum constraint equation (2.24) with any source term $\phi \in(0, \chi]$, the constant $a_{w}^{\wedge}$ must fulfill the inequality

$$
a_{\boldsymbol{w}}^{\wedge} \leqslant(1+\varepsilon) a_{\sigma}^{\wedge}+\left(1+\frac{1}{\varepsilon}\right) a_{\mathcal{L} w}^{\wedge} \leqslant \mathrm{K}_{1 \varepsilon}\|\phi\|_{\infty}^{12}+\mathrm{K}_{2 \varepsilon} .
$$

Thus, for any constant $\chi>0$ and all $\phi \in(0, \chi]$, it holds that

$$
\begin{aligned}
f\left(\chi, \boldsymbol{w}_{\phi}\right) & \geqslant a_{\tau}^{\vee} \chi^{5}+a_{R}^{\vee} \chi-a_{\rho}^{\wedge} \chi^{-3}-\left(\mathrm{K}_{1 \varepsilon}\|\phi\|_{\infty}^{12}+\mathrm{K}_{2 \varepsilon}\right) \chi^{-7} \\
& \geqslant B_{\varepsilon} \chi^{5}+a_{R}^{\vee} \chi-a_{\rho}^{\wedge} \chi^{-3}-\mathrm{K}_{2 \varepsilon} \chi^{-7}
\end{aligned}
$$

where $B_{\varepsilon}:=a_{\tau}^{\vee}-\mathrm{K}_{1 \varepsilon}$. Introduce the rational polynomial on $\chi$ given by

$$
q_{\varepsilon}(\chi):=B_{\varepsilon} \chi^{5}+a_{R}^{\vee} \chi-a_{\rho}^{\wedge} \chi^{-3}-\mathrm{K}_{2 \varepsilon} \chi^{-7} .
$$

We calculate the first and second derivative of $q_{\varepsilon}$ as

$$
\begin{aligned}
& q_{\varepsilon}^{\prime}(\chi)=5 B_{\varepsilon} \chi^{4}+a_{R}^{\vee}+3 a_{\rho}^{\wedge} \chi^{-4}+7 \mathrm{~K}_{2 \varepsilon} \chi^{-8} \\
& q_{\varepsilon}^{\prime \prime}(\chi)=20 B_{\varepsilon} \chi^{3}-12 a_{\rho}^{\wedge} \chi^{-5}-56 \mathrm{~K}_{2 \varepsilon} \chi^{-9}
\end{aligned}
$$

Consider the case (a). In this case, because of the choice $\varepsilon>\frac{\mathrm{k}_{1}}{a_{\tau}^{\vee}-\mathrm{k}_{1}}$, we have $B_{\varepsilon}>0$, and so $q_{\varepsilon}(\chi)>0$ for sufficiently large $\chi$, and $q_{\varepsilon}$ is increasing. The function $q_{\varepsilon}$ has no positive root only if $a_{\rho}^{\wedge}=\mathrm{K}_{2 \varepsilon}=0$. So if $q_{\varepsilon}$ has no positive root, $q_{\varepsilon}(\chi) \geqslant 0$ for all $\chi \geqslant 0$. If $q_{\varepsilon}$ has at least one positive root, denoting by $\phi_{1}$ the largest positive root, $q(\chi) \geqslant 0$ for all $\chi \geqslant \phi_{1}$. Recalling now that any constant $\chi$ satisfies $A_{L} \chi=0$, we conclude that

$$
A_{L} \chi+f\left(\chi, w_{\phi}\right) \geqslant 0 \quad \forall \chi \geqslant \phi_{1}, \forall \phi \in(0, \chi],
$$

implying that $\phi_{+}$is a global super-solution of the Hamiltonian constraint (2.21).

For the case (b), since $B_{\varepsilon}<0$ and $a_{\rho}^{\wedge}$ and $\mathrm{K}_{2 \varepsilon}$ are nonnegative, the first derivative $q_{\varepsilon}^{\prime}(\chi)$ is strictly decreasing for $\chi>0$, and since $q_{\varepsilon}^{\prime}(\phi)>0$ for sufficiently small $\chi>0$ and $q_{\varepsilon}^{\prime}(\chi)<0$ for sufficiently large $\chi>0$, the derivative $q_{\varepsilon}^{\prime}$ has a unique positive root, 
at which the polynomial $q_{\varepsilon}$ attains its maximum over $(0, \infty)$. This maximum is positive if both $a_{\rho}^{\wedge}$ and $\mathrm{K}_{2 \varepsilon}$ are sufficiently small, and hence the polynomial $q_{\varepsilon}$ has two positive roots $\phi_{1} \leqslant \phi_{2}$. Similarly to the above we conclude that

$$
A_{L} \chi+f\left(\chi, \boldsymbol{w}_{\phi}\right) \geqslant 0 \quad \forall \chi \in\left[\phi_{1}, \phi_{2}\right], \forall \phi \in(0, \chi]
$$

implying that $\phi_{+}$is a global super-solution of the Hamiltonian constraint (2.21).

Case (a) of the above lemma has the condition $\mathrm{k}_{1}<a_{\tau}^{\vee}$, which is the near-CMC condition. This condition seems to be present in all non-CMC results to date. The above condition also requires that the extrinsic mean curvature $\tau$ is nowhere zero. Noting that there are solutions even for $\tau \equiv 0$ in some cases (cf. [25]), the condition inf $\tau>0$ appears as a rather strong restriction. We see that case (b) of the above lemma removes this restriction, in exchange for the smallness conditions on $\rho, j$, and $\sigma$. We also need the scalar curvature to be strictly positive, which condition is relaxed in the next subsection to allow any metric in the positive Yamabe class.

In the following lemma, we list some constant sub-solutions. They impose considerable restrictions on the allowable data, which is the main reason to consider non-constant sub-solutions in the next subsection.

Lemma 8 (Global sub-solution). Let $(\mathcal{M}, h)$ be a 3-dimensional, smooth, closed Riemannian manifold with metric $h \in W^{s, p}$. Assume that $a_{\tau} \in L^{\infty}$ and that $a_{R}$ is uniformly bounded from above. We distinguish the following three cases:

(a) If $a_{R}^{\wedge}<0$, then the unique positive root of the polynomial

$$
q(\chi)=a_{\tau}^{\wedge} \chi^{4}+a_{R}^{\wedge}
$$

is a global sub-solution of (2.23).

(b) If $a_{\rho}^{\vee}>0$, then the unique positive root of the polynomial

$$
q_{\rho}(\chi)=a_{\tau}^{\wedge} \chi^{8}+\max \left\{1, a_{R}^{\wedge}\right\} \chi^{4}-a_{\rho}^{\vee},
$$

is a global sub-solution of (2.23).

(c) Let $\phi_{+}>0$ be a global super-solution of the Hamiltonian constraint. Let $a_{\sigma}^{\vee}>$ $\mathrm{k}\left(\phi_{+}\right)$, where $\mathrm{k}$ is as in (5.1). Then, with some $\varepsilon \in\left(\mathrm{k}\left(\phi_{+}\right) / a_{\sigma}^{\vee}, 1\right)$, the unique positive root $\phi_{+}$of the polynomial

$$
q_{\sigma}(\chi)=a_{\tau}^{\wedge} \chi^{12}+\max \left\{1, a_{R}^{\wedge}\right\} \chi^{8}-\mathrm{K}_{\varepsilon},
$$

where $\mathrm{K}_{\varepsilon}:=(1-\varepsilon) a_{\sigma}^{\vee}-\left(\frac{1}{\varepsilon}-1\right) \mathrm{k}\left(\phi_{+}\right)$, is a global sub-solution of (2.23).

Proof. For the proof of (a,b), see e.g. [21]. We give a proof of (c) here.

Let $\chi>0$ be any constant function, and let $\boldsymbol{w} \in \boldsymbol{W}^{1,2 r}$. Then we have

$$
\begin{aligned}
f(\chi, w) & =a_{\tau} \chi^{5}+a_{R} \chi-a_{\rho} \chi^{-3}-a_{w} \chi^{-7} \leqslant a_{\tau}^{\wedge} \chi^{5}+a_{R}^{\wedge} \chi-a_{w}^{\vee} \chi^{-7} \\
& \leqslant a_{\tau}^{\wedge} \chi^{5}+C \chi-a_{w}^{\vee} \chi^{-7}
\end{aligned}
$$

where we have used that $a_{\rho}$ is nonnegative, and introduced the constant $C=\max \left\{1, a_{R}^{\wedge}\right\}$. Given any $\varepsilon>0$, the inequality $2\left|\sigma_{a b}(\mathcal{L} \boldsymbol{w})^{a b}\right| \leqslant \varepsilon \sigma^{2}+\frac{1}{\varepsilon}(\mathcal{L} \boldsymbol{w})^{2}$ implies that

$$
8 a_{\boldsymbol{w}}=\sigma^{2}+(\mathcal{L} \boldsymbol{w})^{2}+2 \sigma_{a b}(\mathcal{L} \boldsymbol{w})^{a b} \geqslant(1-\varepsilon) \sigma^{2}-\left(\frac{1}{\varepsilon}-1\right)(\mathcal{L} \boldsymbol{w})^{2},
$$


hence, taking into account (5.1), for any $\boldsymbol{w} \in \boldsymbol{W}^{1,2 r}$ that is a solution of the momentum constraint equation (2.24) with any source term $\phi \in\left(0, \phi_{+}\right]$, the constant $a_{w}^{\vee}$ must fulfill the inequality

$$
a_{\boldsymbol{w}}^{\vee} \geqslant(1-\varepsilon) a_{\sigma}^{\vee}-\left(\frac{1}{\varepsilon}-1\right) a_{\mathcal{L} \boldsymbol{w}}^{\wedge} \geqslant(1-\varepsilon) a_{\sigma}^{\vee}-\left(\frac{1}{\varepsilon}-1\right) \mathrm{k}\left(\phi_{+}\right)=: \mathrm{K}_{\varepsilon} .
$$

We use the above estimate in (5.6) to get, for any $\boldsymbol{w} \in \boldsymbol{W}^{1,2 r}$ that is a solution of the momentum constraint equation (2.24) with any source term $\phi \in\left(0, \phi_{+}\right]$,

$$
f(\chi, w) \leqslant a_{\tau}^{\wedge} \chi^{5}+C \chi-\mathrm{K}_{\varepsilon} \chi^{-7} .
$$

Because of the choice $\mathrm{k}\left(\phi_{+}\right) / a_{\sigma}^{\vee}<\varepsilon<1$, we have $\mathrm{K}_{\varepsilon}>0$. So with the unique positive root $\chi_{*}$ of

$$
q_{\sigma}(\chi):=a_{\tau}^{\wedge} \chi^{5}+C \chi-\mathrm{K}_{\varepsilon} \chi^{-7},
$$

we have $q_{\sigma}(\chi) \leqslant 0$ for any constant $\chi \in\left(0, \chi_{*}\right]$, establishing the proof.

5.2. Non-constant barriers. All global super-solutions found to date appear to require the near-CMC condition; Lemma 7(b) avoids the near-CMC condition, but it requires the scalar curvature to be strictly positive. The following lemma extends this result to arbitrary metrics in the positive Yamabe class $\mathcal{Y}^{+}(\mathcal{M})$.

Lemma 9 (Global super-solution $h \in \mathcal{Y}^{+}$). Let $(\mathcal{M}, h)$ be a 3-dimensional, smooth, closed Riemannian manifold with metric $h \in W^{s, p}$ in $\mathcal{Y}^{+}(\mathcal{M})$. Assume there exist continuous positive functions $u, \Lambda \in W^{s, p}$ that together satisfy:

$$
-\Delta u+\frac{1}{8} R u=\Lambda>0, \quad u>0 .
$$

Let $0<\mathrm{k}_{3}:=u^{\wedge} / u^{\vee}<\infty$, which is a trivially satisfied Harnack-type inequality. Assume that the estimate (5.1) is satisfied for the solution of the momentum constraint equation for two positive constants $\mathrm{k}_{1}$ and $\mathrm{k}_{2}$, and assume that $a_{\rho}, a_{\sigma} \in L^{\infty}$. If the constants $a_{\rho}^{\wedge}, a_{\sigma}^{\wedge}$, and $\mathrm{k}_{2}$ are sufficiently small, then

$$
\phi_{+}=\beta u, \quad \beta=\left[\frac{\Lambda^{\vee}}{2 \mathrm{k}_{1} \mathrm{k}_{3}^{12}\left(u^{\wedge}\right)^{5}}\right]^{1 / 4}>0,
$$

is a positive global super-solution to the Hamiltonian constraint equation.

Proof. Taking $\phi=\beta u$ with a constant $\beta>0$ in (5.7), gives

$$
-\Delta \phi+a_{R} \phi=\beta\left(-\Delta u+\frac{1}{8} R u\right)=\beta \Lambda .
$$

Then for any $\varphi \in C_{+}^{\infty}$, by using (5.3) with $\mathrm{K}_{1}:=2 \mathrm{k}_{1}$ and $\mathrm{K}_{2}:=2 a_{\sigma}^{\wedge}+2 \mathrm{k}_{2}$, we infer

$$
\begin{aligned}
\left\langle A_{L} \phi+f(\phi, w), \varphi\right\rangle & =\langle\nabla \phi, \nabla \varphi\rangle+\left\langle a_{R} \phi+a_{\tau} \phi^{5}-a_{\rho} \phi^{-3}-a_{w} \phi^{-7}, \varphi\right\rangle \\
& \geqslant\left\langle\beta \Lambda+a_{\tau}^{\vee} \phi^{5}-\left[\mathrm{K}_{1}\left(\phi^{\wedge}\right)^{12}+\mathrm{K}_{2}\right] \phi^{-7}-a_{\rho}^{\wedge} \phi^{-3}, \varphi\right\rangle \\
& \geqslant\left\langle\beta \Lambda+\left[a_{\tau}^{\vee}-\mathrm{K}_{1} \mathrm{k}_{3}^{12}\right] \phi^{5}-\mathrm{K}_{2} \phi^{-7}-a_{\rho}^{\wedge} \phi^{-3}, \varphi\right\rangle \\
& \geqslant\left\langle\beta G\left(\beta, \mathrm{K}_{2}, a_{\rho}\right), \varphi\right\rangle,
\end{aligned}
$$


where

$$
G\left(\beta, \mathrm{K}_{2}, a_{\rho}\right):=\Lambda^{\vee}-\mathrm{K}_{1} \mathrm{k}_{3}^{12} \beta^{4}\left(u^{\wedge}\right)^{5}-\mathrm{K}_{2} \beta^{-8}\left(u^{\wedge}\right)^{-7}-a_{\rho}^{\wedge} \beta^{-4}\left(u^{\wedge}\right)^{-3},
$$

and where we have used the fact that $\phi^{\wedge} / \phi^{\vee}=u^{\wedge} / u^{\vee}=\mathrm{k}_{3}$. Therefore, to ensure $\phi$ is a super-solution we must now pick arguments ensuring $G\left(\beta, \mathrm{K}_{2}, a_{\rho}\right) \geqslant 0$. We first pick $\beta$ as in (5.8) giving

$$
\frac{1}{2} \Lambda^{\vee}=\Lambda^{\vee}-\mathrm{K}_{1} \mathrm{k}_{3}^{12}\left(u^{\wedge}\right)^{5} \beta^{4}>0 .
$$

For this fixed $\beta$, we then pick $\mathrm{K}_{2}$ and $a_{\rho}^{\vee}$, each sufficiently small, so that

$$
\frac{1}{2} \Lambda^{\vee}-\mathrm{K}_{2} \beta^{-8}\left(u^{\wedge}\right)^{-7}-a_{\rho}^{\wedge} \beta^{-4}\left(u^{\wedge}\right)^{-3} \geqslant 0 .
$$

The result then follows.

Remark 3. We now make some remarks about the existence of a pair of positive functions $(u, \Lambda)$ which satisfy the hypotheses of Lemma 9. Let the background metric $h_{a b} \in W^{s, p}$ be in the positive Yamabe class. Then in Theorem 11 in Appendix A.7, for the sub-critical range $1 \leqslant q<5$ we establish the existence of a positive $u \in W^{s, p}$ and a constant $\mu_{q}>0$ satisfying

$$
-8 \Delta u+R u=\mu_{q} u^{q} .
$$

So the pair $\left(u, \frac{1}{8} \mu_{q} u^{q}\right)$ readily satisfies (5.7). In a sense the simplest construction of the near-CMC-free global super-solution in Lemma 9 arises by taking $q=1$; one is then simply using the first eigenfunction of the conformal Laplacian to build the global super-solution.

Alternatively, one can consider a solution to the Yamabe problem

$$
-8 \Delta u+R u=u^{5}, \quad u>0,
$$

which exists for sufficiently smooth metrics in the positive Yamabe class, cf. [31]. This approach is taken for simplicity in [22].

In any case, note that the function $u>0$ that satisfies (5.7) is the conformal factor which transforms $h_{a b}$ into a metric with scalar curvature $R_{u}=8 \Lambda u^{-5}>0$.

We remark that without the near-CMC condition, the only potentially strictly positive term appearing in the nonlinearity of the Hamiltonian constraint is the term involving the scalar curvature $R$. Therefore, global super-solution constructions based on the approach in Lemma 9 are restricted to data in $\mathcal{Y}^{+}(\mathcal{M})$. We extend this observation in the next lemma, which essentially says that in a nonpositive Yamabe class, there is no way to build a positive global super-solution without the near-CMC condition as long as we use a global estimate of type (5.1).

Lemma 10 (Near-CMC condition and $a_{w}$ bounds). Let $(\mathcal{M}, h)$ be a 3-dimensional, smooth, closed Riemannian manifold with metric $h \in W^{s, p}$ in a nonpositive Yamabe class, and let $a_{\tau}$ be continuous. Let $\phi_{+} \in W^{s, p}$ with $\phi_{+}>0$ be a global super-solution to the Hamiltonian constraint equation. We assume that any vector field $\boldsymbol{w} \in \boldsymbol{W}^{1,2 r}$ that is a solution of the momentum constraint equation with a source $\phi \leqslant \phi_{+}$satisfies the estimate

$$
a_{w} \leqslant \mathrm{~K}_{1}\left\|\phi_{+}\right\|_{\infty}^{12}+\mathrm{K}_{2}
$$


with some positive constants $\mathrm{K}_{1}$ and $\mathrm{K}_{2}$. Moreover, we assume that this estimate is sharp in the sense that for any $x \in \mathcal{M}$ there exist an open neighborhood $U \ni x$ and a vector field $\boldsymbol{w} \in \boldsymbol{W}^{1,2 r}$ a solution of the momentum constraint equation with a source $\phi \leqslant \phi_{+}$, such that

$$
a_{w}=\mathrm{K}_{1}\left\|\phi_{+}\right\|_{\infty}^{12}+\mathrm{K}_{2} \quad \text { in } U \text {. }
$$

Then, we have $\mathrm{K}_{1} \leqslant \sup _{\mathcal{M}} a_{\tau}$.

Proof. Since the metric is in a nonpositive Yamabe class, there exists $\tilde{\varphi} \in W_{+}^{2-s, p^{\prime}}$ such that $\left\langle\nabla \phi_{+}, \nabla \tilde{\varphi}\right\rangle+\left\langle a_{R} \phi_{+}, \tilde{\varphi}\right\rangle \leqslant 0$. The collection of all neighborhoods in (5.11) will form an open cover of $\mathcal{M}$, and let $\left\{U_{i}\right\}$ be one of its finite subcovers. Let $\left\{\mu_{i}\right\}$ be a partition of unity subordinate to $\left\{U_{i}\right\}$. Then, by writing $\tilde{\varphi}=\sum_{i} \mu_{i} \tilde{\varphi}$, we can expand the expression $\left\langle\nabla \phi_{+}, \nabla \tilde{\varphi}\right\rangle+\left\langle a_{R} \phi_{+}, \tilde{\varphi}\right\rangle$ into a finite sum, which has at least one non-positive term. Without loss of generality, let us assume $\left\langle\nabla \phi_{+}, \nabla \varphi\right\rangle+\left\langle a_{R} \phi_{+}, \varphi\right\rangle \leqslant 0$ with $\varphi=\mu_{i} \tilde{\varphi}$. With $\boldsymbol{w} \in \boldsymbol{W}^{1,2 r}$ being a vector field that satisfies (5.11) with respect to $U:=U_{i}$, we have

$$
\begin{aligned}
0 & \leqslant\left\langle\nabla \phi_{+}, \nabla \varphi\right\rangle+\left\langle a_{R} \phi_{+}+a_{\tau} \phi_{+}^{5}-a_{w} \phi_{+}^{-7}-a_{\rho} \phi_{+}^{-3}, \varphi\right\rangle \\
& \leqslant\left\langle a_{\tau} \phi_{+}^{5}-a_{w} \phi_{+}^{-7}-a_{\rho} \phi_{+}^{-3}, \varphi\right\rangle \\
& =\left\langle a_{\tau} \phi_{+}^{5}-\left[\mathrm{K}_{1}\left(\phi_{+}^{\wedge}\right)^{12}+\mathrm{K}_{2}\right] \phi_{+}^{-7}-a_{\rho} \phi_{+}^{-3}, \varphi\right\rangle \\
& \leqslant\left(\left[a_{\tau}-\mathrm{K}_{1}\left(\phi_{+}^{\wedge} / \phi_{+}\right)^{12}\right] \phi_{+}^{5}, \varphi\right) .
\end{aligned}
$$

Using partitions of unity we can make the support of $\varphi$ arbitrarily small, from which we conclude that $a_{\tau} \geqslant \mathrm{K}_{1}\left(\phi_{+}^{\wedge} / \phi_{+}\right)^{12} \geqslant \mathrm{~K}_{1}$ at some $x \in \mathcal{M}$.

All of the subsequent barrier constructions below are more or less known. A number of the more technically sophisticated construction techniques we employ below were pioneered by Maxwell in [33]. For completeness, we first construct local super-solutions and then global super-solutions for the near-CMC case.

Lemma 11 (Local super-solution). Let $(\mathcal{M}, h)$ be a 3-dimensional, smooth, closed Riemannian manifold with metric $h \in W^{s, p}$. Let $a_{\tau}, a_{\rho}, a_{w} \in W_{+}^{s-2, p}$, and let one of the following conditions hold:

(a) The metric $h$ is in a non-negative Yamabe class, $a_{\tau} \neq 0$, and $a_{\rho}+a_{w} \neq 0$.

(b) The metric $h$ is in the positive Yamabe class, and $a_{\rho}+a_{w} \neq 0$.

(c) The metric $h$ is conformally equivalent to a metric with scalar curvature $-a_{\tau} \neq 0$, thus in particular the metric is in the negative Yamabe class.

Then, there is a positive (local) super-solution $\phi_{+} \in W^{s, p}$ of the Hamiltonian constraint equation (2.23).

Proof. First we prove (a) and (b). Let $u \in W^{s, p}$ be a (weak) solution to

$$
-\Delta u+\frac{1}{8} R u=\lambda u, \quad u>0,
$$

with a constant $\lambda \geqslant 0$, which exists by Theorem 11 in Appendix A.7, and let $v \in W^{s, p}$ be the solution to

$$
\left\langle u^{2} \nabla v, \nabla \varphi\right\rangle+\left\langle\lambda u^{2} v+a_{\tau} v, \varphi\right\rangle=\left\langle a_{\rho}+a_{w}, \varphi\right\rangle, \quad \forall \varphi \in C^{\infty} .
$$


Since $a_{\tau}, a_{\rho}, a_{w} \in W_{+}^{s-2, p}$ with $s p>3$, we have $v \in W^{s, p} \hookrightarrow L^{\infty}$, and since $\lambda u^{2}+a_{\tau} \neq 0$ and $a_{\rho}+a_{w} \neq 0$, Lemma 35 (maximum principle) in Appendix A.6 implies that $v>0$. Let us define $\phi=\beta u v \in W^{s, p}$ for a constant $\beta>0$. Then for any $\varphi \in C_{+}^{\infty}$ we have

$$
\begin{aligned}
\left\langle A_{L} \phi+f(\phi, w), u \varphi\right\rangle= & \langle\nabla \phi, \nabla(u \varphi)\rangle+\left\langle a_{\tau} \phi^{5}+a_{R} \phi-a_{\rho} \phi^{-3}-a_{w} \phi^{-7}, u \varphi\right\rangle \\
= & \beta\left\langle u^{2} \nabla v, \nabla \varphi\right\rangle+\left\langle\beta \lambda u^{2} v+a_{\tau} u \phi^{5}-a_{\rho} u \phi^{-3}-a_{w} u \phi^{-7}, \varphi\right\rangle \\
= & \left\langle a_{\tau}\left[\beta^{5} u^{6} v^{5}-\beta v\right], \varphi\right\rangle+\left\langle a_{\rho}\left[\beta-\beta^{-3} u^{-2} v^{-3}\right], \varphi\right\rangle \\
& +\left\langle a_{w}\left[\beta-\beta^{-7} u^{-6} v^{-7}\right], \varphi\right\rangle,
\end{aligned}
$$

where the second line is obtained by

$$
\begin{aligned}
\left\langle A_{L} \phi+a_{R} \phi, u \varphi\right\rangle & =\beta\langle\nabla(u v), \nabla(u \varphi)\rangle+\frac{\beta}{8}\langle R u v, u \varphi\rangle \\
& =\beta\langle\nabla u, \nabla(u v \varphi)\rangle+\frac{\beta}{8}\langle R u, u v \varphi\rangle+\beta\langle u \nabla v, u \nabla \varphi\rangle \\
& =\beta\langle\lambda u, u v \varphi\rangle+\beta\left\langle u^{2} \nabla v, \nabla \varphi\right\rangle,
\end{aligned}
$$

and the third line is from (5.12). Now, choosing $\beta>0$ sufficiently large, so that $\beta^{4} u^{6} v^{5}-v$ $\geqslant 0,1-\beta^{-4} u^{-2} v^{-3} \geqslant 0$ and $1-\beta^{-8} u^{-6} v^{-7} \geqslant 0$, we ensure that $\phi$ is a super-solution.

Now, let us consider (c). Let $u>0$ be the conformal factor which transforms $h$ into a metric with scalar curvature $\lambda=-8 a_{\tau}$, i.e., let $u \in W^{s, p}$ be a weak solution to

$$
-\Delta u+\frac{1}{8} R u+a_{\tau} u^{5}=0, \quad u>0 .
$$

If $a_{\rho}=a_{w}=0$, the Hamiltonian constraint equation reduces to the above equation and we can take $u$ as a super-solution (it is even a solution). So we can assume in the following that $a_{\rho}+a_{w} \neq 0$. Let $v \in W^{s, p}$ be the solution to

$$
\left\langle u^{2} \nabla v, \nabla \varphi\right\rangle+\left\langle a_{\tau} v, \varphi\right\rangle=\left\langle a_{\rho}+a_{w}, \varphi\right\rangle, \quad \forall \varphi \in C^{\infty} .
$$

Defining $\phi=\beta u v \in W^{s, p}$ for a constant $\beta>0$, the rest of the proof proceeds superficially in the same way as the above case.

Lemma 12 (Near-CMC global super-solution) Let $(\mathcal{M}, h)$ be a 3-dimensional, smooth, closed Riemannian manifold with metric $h \in W^{s, p}$. Let $a_{\tau}, a_{\rho} \in W_{+}^{s-2, p}$ and $a_{\sigma} \in L_{+}^{\infty}$, and let one of the following conditions hold:

(a) The metric $h$ is in a non-negative Yamabe class, $a_{\tau} \neq 0$, and $a_{\rho}+a_{\sigma} \neq 0$. Let $u \in W^{s, p}$ and $v \in W^{s, p}$ be the solutions to

$$
\begin{aligned}
-\Delta u+\frac{1}{8} R u & =\lambda u, \\
-\nabla\left(u^{2} \nabla v\right)+\left(\lambda u^{2}+a_{\tau}\right) v & =a_{\rho}+a_{\sigma}
\end{aligned}
$$

with a constant $\lambda \geqslant 0$.

(b) The metric $h$ is conformally equivalent to a metric with scalar curvature $-a_{\tau} \neq 0$, thus in particular the metric is in the negative Yamabe class. Let $u \in W^{s, p}$ and $v \in W^{s, p}$ be the solutions to

$$
\begin{aligned}
-\Delta u+\frac{1}{8} R u+a_{\tau} u^{5} & =0, \\
-\nabla\left(u^{2} \nabla v\right)+a_{\tau} v & =a_{\rho}+a_{\sigma} .
\end{aligned}
$$


Assume that the estimate (5.1) holds for the momentum constraint equation, and let $\mathrm{k}_{1}<a_{\tau}^{\vee}\left(\frac{\min u v}{\max u v}\right)^{12}$. Then, for any sufficiently large constant $\beta>0, \phi_{+}=\beta u v$ is a global super-solution of the Hamiltonian constraint equation (2.23).

Proof. We give a proof of (a). The proof of (b) is similar. Proceeding as in the proof of the preceding lemma, for any $\varphi \in C_{+}^{\infty}$ we have

$$
\begin{aligned}
&\left\langle A_{L} \phi+f(\phi, w), u \varphi\right\rangle=\langle\nabla \phi, \nabla(u \varphi)\rangle+\left\langle a_{\tau} \phi^{5}+a_{R} \phi-a_{\rho} \phi^{-3}-a_{w} \phi^{-7}, u \varphi\right\rangle \\
&= \beta\left\langle u^{2} \nabla v, \nabla \varphi\right\rangle+\left\langle\beta \lambda u^{2} v+a_{\tau} u \phi^{5}-a_{\rho} u \phi^{-3}-a_{w} u \phi^{-7}, \varphi\right\rangle \\
& \geqslant \beta\left\langle u^{2} \nabla v, \nabla \varphi\right\rangle+\left\langle\beta \lambda u^{2} v+a_{\tau} u \phi^{5}-a_{\rho} u \phi^{-3}-2\left[a_{\sigma}+a_{\mathcal{L} w}\right] u \phi^{-7}, \varphi\right\rangle \\
&=\left\langle a_{\rho}\left[\beta-\beta^{-3} u^{-2} v^{-3}\right], \varphi\right\rangle+\left\langle a_{\sigma}\left[\beta-2 \beta^{-7} u^{-6} v^{-7}\right], \varphi\right\rangle \\
&+\left\langle a_{\tau}\left[\beta^{5} u^{6} v^{5}-\beta v\right]-2 a_{\mathcal{L} w} u \phi^{-7}, \varphi\right\rangle .
\end{aligned}
$$

Then, choosing $\beta$ sufficiently large, and by using (5.1), with $\theta=u v$ we infer

$$
A_{L} \phi+f(\phi, w) \geqslant\left[a_{\tau}^{\vee}\left(\theta^{\vee}\right)^{5}-2 \mathrm{k}_{1}\left(\theta^{\wedge}\right)^{12}\left(\theta^{\vee}\right)^{-7}\right] \beta^{5}-p(\beta),
$$

where $p(\beta)=a_{\tau}\left(v^{\wedge} / u^{\vee}\right) \beta+2 \mathrm{k}_{2}\left(\theta^{\vee}\right)^{-7} \beta^{-7}$. Now, if we have $\mathrm{k}_{1}<\frac{1}{2} a_{\tau}^{\vee}\left(\theta^{\vee} / \theta^{\wedge}\right)^{12}$, then choosing $\beta$ large enough, we ensure that $\phi$ is a super-solution. If we proceeded as in the proof of Lemma 7, we could remove the factor $\frac{1}{2}$ from the condition $\mathrm{k}_{1}<\frac{1}{2} a_{\tau}^{\vee}\left(\theta^{\vee} / \theta^{\wedge}\right)^{12}$; however, we omit it for clarity.

We now also give some examples of non-constant global sub-solutions $\phi_{-}$which are compatible with $\phi_{+}$above in the sense that $0<\phi_{-} \leqslant \phi_{+}$. Such a pair of compatible sub- and super-solutions are needed to establish existence of solutions to the individual Hamiltonian constraint (Theorem 3), and are also needed again to establish existence of solutions to the coupled system (Theorems 1 and 2).

Lemma 13 (Global sub-solution $\left.h \notin \mathcal{Y}^{-}, \rho \neq \equiv 0\right)$. Let $(\mathcal{M}, h)$ be a 3-dimensional, smooth, closed Riemannian manifold with metric $h \in W^{s, p}$ in a non-negative Yamabe class. Let $a_{\rho}, a_{\tau} \in W_{+}^{s-2, p} \backslash\{0\}$. Then, there exists a positive scalar $\phi_{-} \in W^{s, p}$ such that for any constant $\beta \in(0,1], \beta \phi_{-}$is a global sub-solution of the Hamiltonian constraint equation.

Proof. Let $u \in W^{s, p}$ be a (weak) solution to

$$
-\Delta u+\frac{1}{8} R u=\lambda u, \quad u>0,
$$

with a constant $\lambda \geqslant 0$, which exists by Theorem 11 in Appendix A.7, and let $v \in W^{s, p}$ be the solution to

$$
\left\langle u^{2} \nabla v, \nabla \varphi\right\rangle+\left\langle\lambda u^{2} v+a_{\tau} v, \varphi\right\rangle=\left\langle a_{\rho}, \varphi\right\rangle, \quad \forall \varphi \in C^{\infty} .
$$

Since $a_{\rho}, a_{\tau} \in W_{+}^{s-2, p}$ with $s p>3$, we have $v \in W^{s, p} \hookrightarrow L^{\infty}$, and Lemma 35 (maximum principle) in Appendix A.6 implies that $v>0$. Let us define $\phi=\beta u v \in W^{s, p}$ for a constant $\beta>0$. Then for any $\varphi \in C_{+}^{\infty}$ we have

$$
\begin{aligned}
\left\langle A_{L} \phi+f(\phi, w), u \varphi\right\rangle & \leqslant\left\langle A_{L} \phi, u \varphi\right\rangle+\left\langle a_{\tau} \phi^{5}+a_{R} \phi-a_{\rho} \phi^{-3}, u \varphi\right\rangle \\
& =\beta\left\langle u^{2} \nabla v, \nabla \varphi\right\rangle+\left\langle\beta \lambda u^{2} v+a_{\tau} u^{6}(\beta v)^{5}-a_{\rho} u^{-2}(\beta v)^{-3}, \varphi\right\rangle \\
& =\beta\left\langle a_{\rho}\left[1-u^{-2} v^{-3} \beta^{-4}\right], \varphi\right\rangle+\beta\left\langle a_{\tau}\left[u^{6} v^{5} \beta^{4}-1\right], \varphi\right\rangle,
\end{aligned}
$$


where the second line is obtained by (5.13), and the third line is from (5.16). Now, choosing $\beta>0$ sufficiently small, so that $1-u^{-2} v^{-3} \beta^{-4} \leqslant 0$ and $(\beta v)^{4}-1 \leqslant 0$, we ensure that $\phi$ is a sub-solution.

The following lemma extends Lemma 8(a) to all reasonable metrics in the negative Yamabe class.

Lemma 14 (Global sub-solution $h \in \mathcal{Y}^{-}$). Let $(\mathcal{M}, h)$ be a 3-dimensional, smooth, closed Riemannian manifold with metric $h \in W^{s, p}$ in $\mathcal{Y}^{-}(\mathcal{M})$. In addition, let $a_{\tau} \in W^{s-2, p}$, and let the metric $h$ be conformally equivalent to a metric with scalar curvature $\left(-a_{\tau}\right)$. Then, there exists a positive scalar function $\phi_{-} \in W^{s, p}$ such that for any $\beta \in(0,1], \beta \phi_{-}$is a global sub-solution of the Hamiltonian constraint equation.

Proof. Let $u>0$ be the conformal factor which transforms $h$ into a metric with scalar curvature $\lambda=-8 a_{\tau}$, i.e., let $u \in W^{s-2, p}$ be a weak solution to

$$
-\Delta u+\frac{1}{8} R u+a_{\tau} u^{5}=0, \quad u>0 .
$$

Taking $\phi=\beta u$ with a constant $\beta>0$, we have

$$
\begin{aligned}
A_{L} \phi+f(\phi, w) & \leqslant A_{L} \phi+a_{\tau} \phi^{5}+a_{R} \phi=-\beta \Delta u+a_{\tau}(\beta u)^{5}+\frac{\beta}{8} R u \\
& =\beta a_{\tau} u^{5}\left(\beta^{4}-1\right) .
\end{aligned}
$$

By choosing $\beta \in(0,1]$, we get the sub-solution.

The following lemma shows that the additional condition on the metric appearing in Lemma 14 is indeed not restrictive. It is worth noting that this next result can be viewed as an apparently new non-existence result in the context of the non-CMC constraints, which is interesting in its own right. This result was first proved in [33] for the case of $p=2$; we just need to reinterpret it here in our setting. It states that for there to be a (CMC or non-CMC) solution to the Hamiltonian constraint, the background metric $h_{a b}$ must be conformally equivalent to a metric with scalar curvature equal to $\left(-a_{\tau}\right)$.

Lemma 15 (Non-existence $\left.h \in \mathcal{Y}^{-}\right)$. Let $(\mathcal{M}, h)$ be a 3-dimensional, smooth, closed Riemannian manifold with metric $h \in W^{s, p}$ in $\mathcal{Y}^{-}(\mathcal{M})$. Let $a_{\tau} \in W^{s-2, p}$, and let there exist a solution to the Hamiltonian constraint equation. Then, the metric $h$ is conformally equivalent to a metric with scalar curvature $\left(-a_{\tau}\right)$.

Proof. It suffices to show that the equation

$$
-\Delta \psi+\frac{1}{8} R \psi+a_{\tau} \psi^{5}=0
$$

has a solution $\psi>0$. Since the above equation is just a Hamiltonian constraint equation with $a_{\rho}=a_{w}=0$, Theorem 3 establishes the proof upon constructing sub- and super-solutions to (5.17).

Let $\phi>0$ be a solution to the (general) Hamiltonian constraint equation. Then, since both $a_{\rho}$ and $a_{w}$ are non-negative, we have

$$
-\Delta \phi+\frac{1}{8} R \phi+a_{\tau} \phi^{5} \geqslant 0
$$

which means that $\phi$ is a super-solution to (5.17). 
Let $u \in W^{s, p}$ be a solution to

$$
-\Delta u+\frac{1}{8} R u=-\lambda u, \quad u>0,
$$

with a constant $\lambda>0$, which exists by Theorem 11 in Appendix A.7, and with a real parameter $\varepsilon$, let $v_{\varepsilon} \in W^{s, p}$ be the solution to

$$
\left\langle u^{2} \nabla v_{\varepsilon}, \nabla \varphi\right\rangle+\left\langle\lambda u^{2} v_{\varepsilon}, \varphi\right\rangle=\left\langle\lambda u^{2}-a_{\tau} \varepsilon, \varphi\right\rangle, \quad \forall \varphi \in C^{\infty} .
$$

We have $v_{\varepsilon} \equiv 1$ for $\varepsilon=0$, and we have $v_{\varepsilon} \in W^{s, p} \hookrightarrow L^{\infty}$, so as $\varepsilon$ goes to $0, v_{\varepsilon}$ tends to 1 uniformly. Let us fix $\varepsilon>0$ such that $v_{\varepsilon} \geqslant \frac{1}{2}$. By taking $\psi=\beta u v_{\varepsilon}$ with a constant $\beta>0$, and using (5.13), it holds for any $\varphi \in C_{+}^{\infty}$ that

$$
\begin{aligned}
\langle\nabla \psi, \nabla(u \varphi)\rangle+\left\langle\frac{1}{8} R \psi+a_{\tau} \psi^{5}, u \varphi\right\rangle & =\beta\left\langle u^{2} \nabla v_{\varepsilon}, \nabla \varphi\right\rangle+\left\langle a_{\tau} u^{6}\left(\beta v_{\varepsilon}\right)^{5}-\beta \lambda u^{2} v_{\varepsilon}, \varphi\right\rangle \\
& =\beta\left\langle a_{\tau}\left(u^{6} v_{\varepsilon}^{5} \beta^{4}-\varepsilon\right), \varphi\right\rangle+\beta \lambda\left\langle u^{6}\left(1-2 v_{\varepsilon}\right), \varphi\right\rangle .
\end{aligned}
$$

Now, by choosing $\beta>0$ small enough, we can ensure that $\psi$ is a sub-solution of (5.17).

5.3. A priori $L^{\infty}$ bounds on $W^{1,2}$ solutions. We now establish some related a priori $L^{\infty}$-bounds on any $W^{1,2}$-solution to the Hamiltonian constraint equation. Although such results are standard for semi-linear scalar problems with monotone nonlinearities (for example, see [29]), the nonlinearity appearing in the Hamiltonian constraint becomes non-monotone when $R$ becomes negative. Nonetheless, we are able to obtain a priori $L^{\infty}$-bounds on solutions to the Hamiltonian constraint in all cases including the nonmonotone case. See [21] for an analogue of this result in the case of compact manifolds with boundary; in that case a more general result is possible.

Lemma 16 ((Pointwise a priori bounds). Let $\phi \in W^{1,2}$ be any non-constant positive solution of the Hamiltonian constraint equation (2.23).

(a) Let $a_{\tau R}^{\vee}:=\operatorname{ess} \inf \left(a_{\tau}+a_{R}\right)>0$, and let $a_{\rho}^{\wedge}$ and $a_{w}^{\wedge}$ be finite. Then, $\phi$ satisfies the a priori bound

$$
\phi^{4} \leqslant \max \left\{1, \frac{a_{\rho}^{\wedge}+a_{\boldsymbol{w}}^{\wedge}}{a_{\tau R}^{\vee}}\right\} .
$$

(b) Let $a_{\tau}^{\vee}>0$ and let $a_{\rho}^{\wedge}$ and $a_{w}^{\wedge}$ be finite. Then, $\phi$ satisfies the a priori bound

$$
\phi^{4} \leqslant \max \left\{1, \frac{\sqrt{\left(a_{R}^{\vee}\right)^{2}+a_{\tau}^{\vee}\left(a_{\rho}^{\wedge}+a_{\boldsymbol{w}}^{\wedge}\right)}-a_{R}^{\vee}}{a_{\tau}^{\vee}}\right\} .
$$

(c) Let $a_{\rho w}^{\vee}:=\operatorname{ess} \inf \left(a_{\rho}+a_{w}\right)>0$, and let $a_{\tau}^{\wedge}$ be finite. Then, $\phi$ satisfies the a priori bound

$$
\phi^{4} \geqslant \frac{a_{\rho w}^{\vee}}{\max \left\{a_{\rho w}^{\vee}, a_{\tau}^{\wedge}+a_{R}^{\wedge}\right\}} .
$$


Proof. We will only prove (a) since the other cases can be proven similarly.

Let $\chi \in W^{1,2}$ be any function with $\chi \geqslant 1$. Then for $\varphi \in C_{+}^{\infty}$ we have

$$
\begin{aligned}
\left\langle f_{w}(\chi), \varphi\right\rangle & \geqslant\left(\chi^{\vee}\right)^{5}\left\langle a_{\tau}, \varphi\right\rangle+\chi^{\vee}\left\langle a_{R}, \varphi\right\rangle-\left(\chi^{\vee}\right)^{-3}\left(a_{\rho}, \varphi\right)-\left(\chi^{\vee}\right)^{-7}\left(a_{w}, \varphi\right) \\
& \geqslant\left(a_{\tau R}^{\vee} \chi^{\vee}-\left(\chi^{\vee}\right)^{-3}\left[a_{\rho}^{\wedge}+a_{w}^{\wedge}\right]\right)\|\varphi\|_{1} .
\end{aligned}
$$

So we conclude that

$$
\left\langle f_{w}(\chi), \varphi\right\rangle \geqslant 0 \quad \forall \chi \geqslant \phi^{\wedge}, \quad \chi \in W^{1,2}, \quad \forall \varphi \in C_{+}^{\infty},
$$

where $\left(\phi^{\wedge}\right)^{4}=\max \left\{1, \frac{a_{\rho}^{\wedge}+a_{w}^{\wedge}}{a_{\tau R}^{\vee}}\right\}$.

Now, suppose that $\phi \in W^{1,2}$ is a solution of the Hamiltonian constraint equation, such that $\phi \& \phi^{\wedge}$. Denoting by $\left(\phi-\phi^{\wedge}\right)^{+}$the positive part of $\phi-\phi^{\wedge}$ (cf. Appendix A.6), then we have

$$
\begin{aligned}
0 & \geqslant-\left\langle f_{w}(\phi),\left(\phi-\phi^{\wedge}\right)^{+}\right\rangle=\left(\nabla \phi, \nabla\left(\phi-\phi^{\wedge}\right)^{+}\right)=\left(\nabla\left(\phi-\phi^{\wedge}\right)^{+}, \nabla\left(\phi-\phi^{\wedge}\right)^{+}\right) \\
& \geqslant c\left\|\left(\phi-\phi^{\wedge}\right)^{+}-\overline{\left(\phi-\phi^{\wedge}\right)^{+}}\right\|_{2}^{2},
\end{aligned}
$$

where $c>0$, and $\overline{\left(\phi-\phi^{\wedge}\right)^{+}}$is the integral average of $\left(\phi-\phi^{\wedge}\right)^{+}$. This implies that $\phi$ is constant, leading to a contradiction.

\section{Proof of the Main Results}

It is convenient to prove Theorem 2 first, which is the most general of the three; the proofs of Theorem 1 and Theorem 3 involve minor modifications of the proof of Theorem 2.

6.1. Proof of Theorem 2. Our strategy will be to prove the theorem first for the case $s \leqslant 2$, and then to bootstrap to include the higher regularity cases.

Step 1. The choice of function spaces. We have the (reflexive) Banach spaces $X=W^{s, p}$ and $Y=W^{e, q}$, where $p, q \in(3, \infty), s=s(p) \in\left(1+\frac{3}{p}, 2\right]$, and $e=e(p, s, q) \in$ $(1, s] \cap\left(1+\frac{3}{q}, s-\frac{3}{p}+\frac{3}{q}\right]$. We have the ordered Banach space $Z=W^{\tilde{s}, p}$ with the compact embedding $X=W^{s, p} \hookrightarrow W^{\tilde{s}, p}=Z$, for $\tilde{s} \in\left(\frac{3}{p}, s\right)$. The interval $\left[\phi_{-}, \phi_{+}\right]_{\tilde{s}, p}$ is nonempty (by compatibility of the barriers we will choose below), and by Lemma 1 at the end of Sect. 3 it is also convex with respect to the vector space structure of $W^{\tilde{s}, p}$ and closed with respect to the norm topology of $W^{\tilde{s}, p}$. We then take $U=\left[\phi_{-}, \phi_{+}\right]_{\tilde{s}, p} \cap \bar{B}_{M}$ for sufficiently large $M$ (to be determined below), where $\bar{B}_{M}$ is the closed ball in $Z=W^{\tilde{s}, p}$ of radius $M$ about the origin, ensuring that $U$ is non-empty, convex, closed, and bounded as a subset of $Z=W^{\tilde{s}, p}$.

Step 2. Construction of the mapping $S$. We have $\boldsymbol{b}_{j} \in \boldsymbol{W}^{e-2, q}$, and $\boldsymbol{b}_{\tau} \in \boldsymbol{L}^{z}$ with $z=\frac{3 q}{3+(2-e) q}$ so that $\boldsymbol{L}^{z} \hookrightarrow \boldsymbol{W}^{e-2, q}$. Moreover, since the metric admits no conformal Killing field, by Lemma 6 the momentum constraint equation is uniquely solvable for any "source" $\phi \in\left[\phi_{-}, \phi_{+}\right]_{\tilde{s}, p}$. The ranges for the exponents ensure that Lemma 2 holds, so that the momentum constraint solution map

$$
S:\left[\phi_{-}, \phi_{+}\right]_{\tilde{s}, p} \rightarrow \boldsymbol{W}^{e, q}=Y,
$$

is continuous. 
Step 3. Construction of the mapping $T$. Define $r=\frac{3 p}{3+(2-s) p}$, so that the continuous embedding $L^{r} \hookrightarrow W^{s-2, p}$ holds. Since the pointwise multiplication is bounded on $L^{2 r} \otimes L^{2 r} \rightarrow L^{r}$, and $\boldsymbol{w} \in \boldsymbol{W}^{e, q} \hookrightarrow \boldsymbol{W}^{1,2 r}$, we have $a_{\boldsymbol{w}} \in W^{s-2, p}$ by $\sigma \in L^{2 r}$. The embeddings $W^{1, z} \hookrightarrow W^{e-1, q} \hookrightarrow L^{2 r}$ also guarantee that $a_{\tau}=\frac{1}{12} \tau^{2} \in W^{s-2, p}$. We have the scalar curvature $R \in W^{s-2, p}$, and these considerations show that the Hamiltonian constraint equation is well defined with $\left[\phi_{-}, \phi_{+}\right]_{s, p}$ as the space of solutions.

Suppose for the moment that the scalar curvature $R$ of the background metric $h$ is continuous, and by using the map $T^{s}$ introduced in Lemma 3, define the map $T$ by $T(\phi, w)=T^{s}\left(\phi, a_{w}\right)$, where $a_{w}$ is now considered as an expression depending on $\boldsymbol{w}$. Then Lemma 3 implies that the map $T:\left[\phi_{-}, \phi_{+}\right]_{\tilde{s}, p} \times \boldsymbol{W}^{e, q} \rightarrow W^{s, p}$ is continuous for any reasonable shift $a_{s}$, which, by Lemma 4, can be chosen so that $T$ is monotone in the first variable. Combining the monotonicity with Lemma 5, we infer that the interval $\left[\phi_{-}, \phi_{+}\right]_{\tilde{s}, p}$ is invariant under $T\left(\cdot, a_{\boldsymbol{w}}\right)$ if $\boldsymbol{w} \in S\left(\left[\phi_{-}, \phi_{+}\right]_{\tilde{s}, p}\right)$. Since $\boldsymbol{L}^{z} \hookrightarrow \boldsymbol{W}^{e-2, q}$, from Theorem 6 we have

$$
\|\boldsymbol{w}\|_{e, q} \leqslant C\left\|\boldsymbol{b}_{\tau} \phi^{6}+\boldsymbol{b}_{j}\right\|_{e-2, q} \leqslant C\left\|\phi_{+}\right\|_{\infty}^{6}\left\|\boldsymbol{b}_{\tau}\right\|_{z}+C\left\|\boldsymbol{b}_{j}\right\|_{e-2, q}
$$

for any $\boldsymbol{w} \in S\left(\left[\phi_{-}, \phi_{+}\right]_{\tilde{s}, p}\right)$. In view of Lemma 6 , this shows that there exists a closed ball $\bar{B}_{M} \subset W^{\tilde{s}, p}$ such that

$$
\phi \in\left[\phi_{-}, \phi_{+}\right]_{\tilde{s}, p} \cap \bar{B}_{M}, \quad \boldsymbol{w} \in S\left(\left[\phi_{-}, \phi_{+}\right]_{\tilde{s}, p} \cap \bar{B}_{M}\right) \Rightarrow T(\phi, w) \in \bar{B}_{M} .
$$

Under the conditions in the above displayed formula, from the invariance of the interval $\left[\phi_{-}, \phi_{+}\right]_{\tilde{s}, p}$, we indeed have $T(\phi, w) \in U=\left[\phi_{-}, \phi_{+}\right]_{\tilde{s}, p} \cap \bar{B}_{M}$.

However, the scalar curvature of $h$ may be not continuous, and in general it is not clear how to introduce a shift so that the resulting operator is monotone. Nevertheless, we can conformally transform the metric into a metric with continuous scalar curvature, cf. Theorem 12, and by using the conformal covariance of the Hamiltonian constraint, we will be able to construct an appropriate mapping $T$. Let $\tilde{h}=\theta^{4} h$ be a metric with continuous scalar curvature, where $\theta \in W^{s, p}$ is the (positive) conformal factor of the scaling. Let $\tilde{T}^{s}$ be the mapping introduced in Lemma 3, corresponding to the Hamiltonian constraint equation with the background metric $\tilde{h}$, and the coefficients $\tilde{a}_{\tau}=a_{\tau}$, and $\tilde{a}_{\rho}=\theta^{-8} a_{\rho}$. With $\tilde{a}_{w}=\theta^{-12} a_{w}$, this scaled Hamiltonian constraint equation has sub- and super-solutions $\theta^{-1} \phi_{-}$and $\theta^{-1} \phi_{+}$, respectively, as long as $\phi_{-}$and $\phi_{+}$are sub- and super-solutions respectively of the original Hamiltonian constraint equation, cf. Appendix A.8. We choose the shift in $\tilde{T}^{s}$ so that it is monotone in $\left[\theta^{-1} \phi_{-}, \theta^{-1} \phi_{+}\right]_{\tilde{s}, p}$. Then by the monotonicity and the above mentioned sub- and super-solution property under conformal scaling, for $\boldsymbol{w} \in S\left(\left[\phi_{-}, \phi_{+}\right]_{\tilde{s}, p}\right), \tilde{T}^{s}\left(\cdot, \theta^{-12} a_{w}\right)$ is invariant on $\left[\theta^{-1} \phi_{-}, \theta^{-1} \phi_{+}\right]_{\tilde{s}, p}$. Finally, we define

$$
T(\phi, \boldsymbol{w})=\theta \tilde{T}^{s}\left(\theta^{-1} \phi, \theta^{-12} a_{\boldsymbol{w}}\right),
$$

where, as before, $a_{\boldsymbol{w}}$ is considered as an expression depending on $\boldsymbol{w}$. From the pointwise multiplication properties of $\theta$ and $\theta^{-1}$, the map $T:\left[\phi_{-}, \phi_{+}\right]_{\tilde{s}, p} \times \boldsymbol{W}^{e, q} \rightarrow W^{s, p}$ is continuous, and from the monotonicity and Lemma $6, T(\cdot, \boldsymbol{w})$ is invariant on $U=$ $\left[\phi_{-}, \phi_{+}\right]_{\tilde{s}, p} \cap \bar{B}_{M}$ for $\boldsymbol{w} \in S(U)$, where $M$ is taken to be sufficiently large. Moreover, if the fixed point equation

$$
\phi=\theta \tilde{T}^{s}\left(\theta^{-1} \phi, \theta^{-12} a_{w}\right)
$$


is satisfied, then $\theta^{-1} \phi$ is a solution to the scaled Hamiltonian constraint equation with $\tilde{a}_{w}=\theta^{-12} a_{w}$, and so by conformal covariance, $\phi$ is a solution to the original Hamiltonian constraint equation, cf. Appendix A.8.

Step 4. Barrier choices and application of the fixed point theorem. At this point, Theorem 5 implies the Main Theorem 2, provided that we have an admissible pair of barriers for the Hamiltonian constraint. The ranges for the exponents ensure through Corollary 1 that we can use the estimate (5.1); see the discussion following the estimate at the beginning of Sect. 5. We will separate into the two cases in the theorem, depending on which Yamabe class we are in:

(a) $\quad h_{a b}$ is in $\mathcal{Y}^{-}(\mathcal{M})$ : We use the global constant super-solution from Lemma 7(a) or the non-constant super-solution from Lemma 12 depending on whether $\rho$ and $\sigma$ are both in $L^{\infty}$, and the global sub-solution from Lemma 14.

(b) $\quad h_{a b}$ is in $\mathcal{Y}^{0}(\mathcal{M})$ or in $\mathcal{Y}^{+}$: We use the global constant super-solution from Lemma 7(a) or the non-constant super-solution from Lemma 12 depending on whether $\rho$ and $\sigma$ are both in $L^{\infty}$, and the global sub-solution from Lemma 13 or Lemma 8(c).

This concludes the proof for the case $s \leqslant 2$.

Step 5: Bootstrap. Now suppose that $s>2$. First of all we need to show that the equations are well defined in the sense that the involved operators are bounded in appropriate spaces. All other conditions being obviously satisfied, we will show that $a_{\tau} \in W^{s-2, p}$, and $a_{\boldsymbol{w}} \in W^{s-2, p}$ for any $\boldsymbol{w} \in \boldsymbol{W}^{e, q}$. Since $\tau, \sigma$ and $\mathcal{L} \boldsymbol{w}$ belong to $W^{e-1, q}$, it suffices to show that the pointwise multiplication is bounded on $W^{e-1, q} \otimes W^{e-1, q} \rightarrow W^{s-2, p}$, and by employing Corollary 3(b) in the Appendix, we are done as long as $s-2 \leqslant e-1 \geqslant 0$, $s-2-\frac{3}{p}<2\left(e-1-\frac{3}{q}\right)$, and $s-2-\frac{3}{p} \leqslant e-1-\frac{3}{q}$. After a rearrangement these conditions read: $e \geqslant 1, e \geqslant s-1, e>\frac{3}{q}+\frac{d}{2}$, and $e \geqslant \frac{3}{q}+d-1$, with the shorthand $d=s-\frac{3}{p}>1$, the latter inequality by the hypothesis of the theorem. We have $d-1>\frac{d}{2}$ for $d>2$, and $1 \geqslant \frac{d}{2}$ for $d \leqslant 2$, meaning that the condition $e>\frac{3}{q}+\frac{d}{2}$ is implied by the hypotheses $e \geqslant \frac{3}{q}+d-1$ and $e>1+\frac{3}{q}$. So we conclude that the constraint equations are well defined.

Next, we will treat the equations as equations defined with $s=e=2$ and with $p$ and $q$ appropriately chosen. This is possible, since if the quadruple $(p, s, q, e)$ satisfies the hypotheses of the theorem, then $(\tilde{p}, \tilde{s}=2, \tilde{q}, \tilde{e}=2)$ satisfies the hypotheses too, provided that $2-\frac{3}{\tilde{p}} \leqslant s-\frac{3}{p}$, and $1<2-\frac{3}{\tilde{q}} \leqslant e-\frac{3}{q}$. Since the latter conditions reflect the Sobolev embeddings $W^{s, p} \hookrightarrow W^{2, \tilde{p}}$ and $W^{e, q} \hookrightarrow W^{2, \tilde{q}} \hookrightarrow W^{1, \infty}$, the coefficients of the equations can also be shown to satisfy sufficient conditions for posing the problem for $(\tilde{p}, 2, \tilde{q}, 2)$. Finally, we have $\tau \in W^{e-1, q} \hookrightarrow W^{1, \tilde{q}}=W^{1, z}$ since $z=\tilde{q}$ by $\tilde{e}=2$ for this new formulation. Now, by the special case $s \leqslant 2$ of this theorem that is proven in the above steps, under the remaining hypotheses including the conditions on the metric and the near-CMC condition, we have $\phi \in W^{2, \tilde{p}}$ with $\phi>0$ and $\boldsymbol{w} \in \boldsymbol{W}^{2, \tilde{q}}$ solution to the coupled system.

To complete the proof we only need to show that these solutions indeed satisfy $\phi \in$ $W^{s, p}$ and $\boldsymbol{w} \in \boldsymbol{W}^{e, q}$. Suppose that $\phi \in W^{s_{1}, p_{1}}$ and $\boldsymbol{w} \in \boldsymbol{W}^{e_{1}, q_{1}}$, with $1<s_{1}-\frac{3}{p_{1}} \leqslant s-\frac{3}{p}$, $1<e_{1}-\frac{3}{q_{1}} \leqslant e-\frac{3}{q}, \max \{2, s-2\} \leqslant s_{1} \leqslant s$, and $\max \{2, e-2\} \leqslant e_{1} \leqslant \min \{e, s\}$. Then we have $\boldsymbol{b}_{\tau} \phi^{6}+\boldsymbol{b}_{j} \in \boldsymbol{W}^{e-2, q}$, and so Corollary 5 from Appendix A.5 implies that $\boldsymbol{w} \in \boldsymbol{W}^{e, q}$. This implies that $a_{\boldsymbol{w}} \in W^{s-2, p}$, and by employing Corollary 5 once again, we get $\phi \in W^{s, p}$. The proof is completed by induction. 
6.2. Proof of Theorem 1. The proof is identical to the proof of Theorem 2, except for the particular barriers used. In the proof of Theorem 2, the near-CMC condition is used to construct global barriers satisfying

$$
0<\phi_{-} \leqslant \phi_{+}<\infty
$$

for all three Yamabe classes, and then the supporting results for the operators $S$ and $T$ established in Sect. 4.1 and Sect. 4.2 are used to reduce the proof to invoking Theorem 5. The construction of $\phi_{+}$is in fact the only place in the proof of Theorem 2 that requires the near-CMC condition. Here, the proof is identical, except that the additional conditions made on the background metric $h_{a b}$ (that it be in $\mathcal{Y}^{+}(\mathcal{M})$ ), and on the data (the smallness conditions on $\sigma, \rho$, and $j$ ) allow us to make use of the alternative construction of a global super-solution given in Lemma 9, together with compatible global sub-solution given in Lemma 13, properly scaled for compatibility with the super-solution. Theorem 1 now follows from Theorem 5, without the use of near-CMC conditions.

6.3. Proof of Theorem 3. The CMC result in this theorem can be proved using the same analysis framework used for the proofs of the two non-CMC results in Theorem 1 and Theorem 2 above. Therefore, the proof follows the same general outline of the proof of Theorem 2, with slightly different spaces and supporting results. The main difference is that we can avoid having to construct "global" barriers and getting uniform bounds on the solution to the momentum constraint, since it is solved only once a priori and then is input as data into the nonlinearity of the Hamiltonian constraint.

The case (d) follows from the Yamabe classification, cf. Appendix A.7.

Since otherwise we can use the conformal covariance of the Hamiltonian constraint as in Sect. 6.1, for simplicity, assume that the scalar curvature of the background metric is continuous. Also assume that $s \leqslant 2$, and let us look at the hypotheses of Theorem 5 . We have the (reflexive) Banach spaces $X=W^{s, p}$ and $Y=W^{1,2 r}$, where $p \in\left(\frac{3}{2}, \infty\right)$, $s=s(p) \in\left(\frac{3}{p}, \infty\right) \cap[1,2]$, and $r=r(s, p)=\frac{3 p}{3+(2-s) p}$. On the diagram in Fig. 2, for $s \leqslant 2$ the space $\boldsymbol{W}^{1,2 r}$ corresponds to the lower right corner of the shaded parallelogram, and so $\boldsymbol{W}^{1,2 r}$ contains all the spaces $\boldsymbol{W}^{e, q}$ which are represented by the points in the shaded parallelogram. In fact, $\boldsymbol{W}^{1,2 r}$ is outside of this parallelogram, because of the strict inequality relating $e$ and $q$ in order to have the boundedness of the pointwise multiplication on $W^{e-1, q} \otimes W^{e-1, q} \rightarrow W^{s-2, p}$ by using Corollary 3(b). However, the conditions of Corollary 3(b) are not necessary conditions when some of the smoothness indices are integers, for example, in our case the pointwise multiplication is bounded on $L^{2 r} \otimes L^{2 r} \rightarrow L^{r}$, even though these spaces do not satisfy the conditions of the corollary. As a consequence, as we have seen e.g. in Sect. 2.4, the constraint equations are well defined for these spaces.

We have the ordered Banach space $Z=W^{\tilde{s}, p}$ with the compact embedding $X=$ $W^{s, p} \hookrightarrow W^{\tilde{s}, p}=Z$, for $\tilde{s} \in\left(\frac{3}{p}, s\right)$. The interval $\left[\phi_{-}, \phi_{+}\right]_{\tilde{s}, p}$ is nonempty (by compatibility of the barriers we will choose below), and by Lemma 1 at the end of Sect. 3 it is also convex with respect to the vector space structure of $W^{\tilde{s}, p}$ and closed with respect to the norm topology of $W^{\tilde{s}, p}$. We then take $U=\left[\phi_{-}, \phi_{+}\right]_{\tilde{s}, p} \cap \bar{B}_{M}$ for sufficiently large $M$ (to be determined below), where $\bar{B}_{M}$ is the closed ball in $Z=W^{\tilde{s}, p}$ of radius $M$ about the origin, ensuring that $U$ is non-empty, convex, closed, and bounded as a subset of $Z=W^{\tilde{s}, p}$.

We take as $T$ the shifted Picard mapping $T^{s}$ having as its fixed-point a solution to the Hamiltonian constraint, and we take $S(\phi)=\boldsymbol{w}=-A_{\mathbb{L}}^{-1} \boldsymbol{b}_{j} \in \boldsymbol{W}^{1,2 r}$, which is indepen- 
dent of $\phi$, since the momentum equation decouples from the Hamiltonian constraint in this case. The map $S$, which is constant as a function of $\phi$ due to the CMC de-coupling, is trivially continuous as a map $S: U \rightarrow \boldsymbol{W}^{1,2 r}=Y$. We now consider properties we have for $T$. By Lemma 3, $T: U \times \mathcal{R}(S) \rightarrow W^{s, p}=X$ is a continuous map. By Lemma 4, $T$ is invariant on the closed interval $\left[\phi_{-}, \phi_{+}\right]_{\tilde{s}, p}$, and by Lemma $6, T$ is invariant on $U=\left[\phi_{-}, \phi_{+}\right]_{\tilde{s}, p} \cap \bar{B}_{M}$. To summarize, $T$ is invariant on the non-empty, closed, convex, bounded set $U$.

Finally, Theorem 5 implies the Main Theorem 3, as long as we have an admissible pair of barriers for the Hamiltonian constraint. That is when we need to separate into the three remaining cases in the theorem, depending on which Yamabe class we are in:

(a) $\quad h_{a b}$ is in $\mathcal{Y}^{-}(\mathcal{M}) ; \tau \neq 0$ : We take the super-solution from Lemma 11(c), and we take the sub-solution from Lemma 14. These lemmata require that the metric $h_{a b}$ is conformally equivalent to a metric with scalar curvature $\left(-a_{\tau}\right)$, and we shall verify this condition. By conformal invariance, it suffices to verify the condition for metrics with continuous and negative scalar curvature, meaning that we have to solve Eq. (5.17) with $R<0$ continuous and $a_{\tau}>0$ constant. Indeed, this equation has a positive solution $\psi \in W^{s, p}$ as the constants $\psi_{-}=\left(\frac{\min |R|}{8 a_{\tau}}\right)^{1 / 4}$ and $\psi_{+}=\left(\frac{\max |R|}{8 a_{\tau}}\right)^{1 / 4}$ are respectively sub- and super-solutions of (5.17).

(b) $h_{a b}$ is in $\mathcal{Y}^{+}(\mathcal{M}) ; \rho \neq 0$ or $\sigma \neq 0$ : We take the super-solution from Lemma 11(b), and we take the sub-solution from Lemma 13. For the case $\rho=0$ and $\sigma \neq 0$, a local sub-solution can easily be constructed following the approach in the proof of Lemma 13.

(c) $h_{a b}$ is in $\mathcal{Y}^{0}(\mathcal{M}) ; \tau \neq 0 ; \rho \neq 0$ or $\sigma \neq 0$ : We take the super-solution from Lemma 11(a), and we take the sub-solution from Lemma 13. The case $\rho=0$ and $\sigma \neq 0$ is treated as above.

To complete the proof one can bootstrap as in Sect. 6.1.

\section{Summary}

We began in Sect. 2 by summarizing the conformal decomposition of Einstein's constraint equations introduced by Lichnerowicz and York, on a closed manifold. After this setting up of the notation, we gave an overview of our main results in Sect. 3, represented by three new weak solution existence results for the Einstein constraint equations in the far-from-CMC, near-CMC, and CMC cases. In Sect. 4 we then developed the necessary results we need for the individual constraint equations in order to analyze the coupled system. In particular, in Sect. 4.1, we first developed some basic technical results for the momentum constraint operator under weak assumptions on the problem data. We also established the properties we need for the momentum constraint solution mapping $S$ appearing in the analysis of the coupled system. In Sect. 4.2, we assumed the existence of barriers $\phi_{-}$and $\phi_{+}$(weak sub- and super-solutions) to the Hamiltonian constraint equation, forming a nonempty positive bounded interval, and then established the properties we need for the Hamiltonian constraint Picard mapping $T$ appearing in the analysis of the coupled system. We then derived several weak global sub- and super-solutions in Sect. 5, based both on constants and on more complex non-constant constructions. While the sub-solutions are similar to those found previously in the literature, some of the super-solutions were new. In particular, we gave two super-solution constructions that do not require the near-CMC condition. The first was constant, and requires that the 
scalar curvature be strictly globally positive. The second was based on a scaled solution to a Yamabe-type problem, and is valid for any background metric in the positive Yamabe class.

In Sect. 6, we proved the main results. In particular, using topological fixed-point arguments and global barrier constructions, we combined the results for the individual constraints and the global barriers to establish existence of coupled non-CMC weak solutions with (positive) conformal factor $\phi \in W^{s, p}$, where $p \in(1, \infty)$ and $s(p) \in\left(1+\frac{3}{p}, \infty\right)$. In the CMC case, the regularity can be reduced to $p \in(1, \infty)$ and $s(p) \in\left(\frac{3}{p}, \infty\right) \cap[1, \infty)$. In the case of $s=2$, we reproduce the CMC existence results of Choquet-Bruhat [10], and in the case $p=2$, we reproduce the CMC existence results of Maxwell [33], but with a different proof; our CMC proof goes through the same analysis framework that we use to obtain the non-CMC results (Theorems 4 and 5). We also assembled a number of new supporting technical results in the body of the paper and in several appendices, including: topological fixed-point arguments designed for the Einstein constraints; construction and properties of general Sobolev classes $W^{s, p}$ and elliptic operators on closed manifolds with weak metrics; the development of a very weak solution theory for the momentum constraint; a priori $L^{\infty}$-estimates for weak $W^{1,2}$-solutions to the Hamiltonian constraint; Yamabe classification of non-smooth metrics in general Sobolev classes $W^{s, p}$; and a discussion and analysis of conformal covariance and the connection between conformal rescaling and the near-CMC condition.

An important feature of the results we presented here is the absence of the near-CMC assumption in the case of the rescaled background metric in the positive Yamabe class, as long as the freely specifiable part of the data given by the matter fields (if present) and the traceless-transverse part of the rescaled extrinsic curvature are taken to be sufficiently small. In this case, the mean extrinsic curvature can be taken to be an arbitrary smooth function without restrictions on the size of its spatial derivatives, so that it can be arbitrarily far from constant. Under these conditions, we have the first existence result for non-CMC solutions without the near-CMC condition. The two advances in the analysis of the Einstein constraint equations that make these results possible were: A topological fixed-point theorem based on compactness arguments that is free of the near-CMC condition (Theorems 4 and 5 and in [21]), and a new construction of global super-solutions for the Hamiltonian constraint that is similarly free of the near-CMC condition (Lemma 7 and Lemma 9). We note that the near-CMC-free constructions based on scaled solutions to a Yamabe-like problem also work for compact manifolds with boundary and other cases; see e.g. [21].

Finally, we point out that our results here and in [21,22] can be viewed as reducing the remaining open questions of existence of non-CMC (weak and strong) solutions without near-CMC conditions to two more basic and clearly stated open problems: (1) Existence of near-CMC-free global super-solutions for the Hamiltonian constraint equation when the background metric is in the non-positive Yamabe classes and for large data; and (2) existence of near-CMC-free global sub-solutions for the Hamiltonian constraint equation when the background metric is in the positive Yamabe class in vacuum (without matter). However, an important new development, which occurred a few months after the first draft of this article was made available, is that Maxwell has now shown [36] how a related topological fixed-point argument can be constructed so that a global subsolution is not needed, as long as the global super-solution is available; this allows for the extension of the far-CMC results in this article to the vacuum case without having to solve problem (2). 
Acknowledgement. The authors would like to thank Jim Isenberg, David Maxwell, and Daniel Pollack for many very insightful comments and suggestions about this work. We would like to thank David Maxwell in particular for his careful reading of earlier drafts of this work and for pointing out various errors. MH was supported in part by NSF Awards 0715146, 0411723, and 0511766, and DOE Awards DE-FG02-05ER25707 and DE-FG02-04ER25620. GN and GT were supported in part by NSF Awards 0715146 and 0411723.

\section{A. Some Key Technical Tools and Some Supporting Results}

A.1. Topological fixed-point theorems. In this Appendix, we give a brief review of some standard topological fixed-point theorems in Banach spaces that provide the framework for our analysis of the coupled constraint equations. The analysis framework that was developed earlier in [26] for analyzing the coupled constraints was based on $k$-contractive mappings, and as a result required the near-CMC condition in order to establish $k$-contractivity. All subsequent non-CMC results (see e.g. [1]) are based on the framework from [26], and as a result remain limited to the near-CMC case. Our interest here is on more general topological fixed-point arguments that will allow us to avoid the near-CMC condition.

Brouwer, Schauder, and Leray-Schauder Fixed-Point Theorems. To establish the main abstract results we will need, we first give a brief overview of some standard results on topological fixed-point arguments involving compactness.

Theorem 7 (Brouwer Theorem). Let $U \subset \mathbb{R}^{n}$ be a non-empty, convex, compact subset, with $n \geqslant 1$. If $T: U \rightarrow U$ is a continuous mapping, then there exists a fixed-point $u \in U$ such that $u=T(u)$.

Proof. See Proposition 2.6 in [54]; a short proof can be based on homotopy-invariance of topological degree.

Theorem 8 (Schauder Theorem). Let $X$ be a Banach space, and let $U \subset X$ be a nonempty, convex, compact subset. If $T: U \rightarrow U$ is a continuous operator, then there exists a fixed-point $u \in U$ such that $u=T(u)$.

Proof. This is a direct extension of the Brouwer Fixed-Point Theorem from $\mathbb{R}^{n}$ to $X$; see Corollary 2.13 in [54]. The short proof involves a simple finite-dimensional approximation algorithm and a limiting argument, extending the Brouwer Fixed-Point Theorem (itself generally having a more complicated proof) from $\mathbb{R}^{n}$ to $X$.

Theorem 9 (Schauder Theorem B). Let $X$ be a Banach space, and let $U \subset X$ be a non-empty, convex, closed, bounded subset. If $T: U \rightarrow U$ is a compact operator, then there exists a fixed-point $u \in U$ such that $u=T(u)$.

Proof. See Theorem 2.A in [54]; the proof is a simple consequence of Theorem 8 above.

A.2. Ordered Banach spaces. These notes follow the main ideas and definitions given in Chap. 7.1, p. 275, in [54], while some examples were taken from [2 and 16]. Let $X$ be a Banach space, $\mathbb{R}_{+}$be the non-negative real numbers. A subset $C \subset X$ is a cone iff given any $x \in C$ and $a \in \mathbb{R}_{+}$the element $a x \in C$. A subset $X_{+} \subset X$ is an order cone iff the following properties hold:

(i) The set $X_{+}$is non-empty, closed, and $X_{+} \neq\{0\}$; 
(ii) Given any $a, b \in \mathbb{R}_{+}$and $x, \underline{x} \in X_{+}$then $a x+b \underline{x} \in X_{+}$;

(iii) If $x \in X_{+}$and $-x \in X_{+}$, then $x=0$.

The second property above says that every order cone is in fact a cone, and that the set $X_{+}$is convex. The space $X=\mathbb{R}^{2}$ is a convenient Banach space to picture non-trivial examples of cones and order cones, as can be seen in Fig. 3. A pair $X, X_{+}$is called an ordered Banach space iff $X$ is a Banach space and $X_{+} \subset X$ is an order cone. The reason for this name is that the order cone $X_{+}$defines several relations on elements in $X$, called order relations, as follows:

$$
\begin{aligned}
u \geqslant v \text { iff } u-v \in X_{+}, & u>v \text { iff } u \geqslant v \text { and } u \neq v, \\
u \gg v \text { iff } u-v \in \operatorname{int}\left(X_{+}\right), & u \neq v \text { iff } u \geqslant v \text { is false; }
\end{aligned}
$$

finally the notations $u \leqslant v, u<v$, and $u \ll v$ are used to mean $v \geqslant u, v>u, v \gg u$, respectively. A simple example of an ordered Banach space is $\mathbb{R}$ with the usual order. Another example can be constructed when this order on $\mathbb{R}$ is transported into $C^{0}(\mathcal{M})$, the set of scalar-valued functions on a set $\mathcal{M} \subset \mathbb{R}^{n}$, with $n \geqslant 1$. An order on $C^{0}(\mathcal{M})$ is the following: the functions $u, v \in C^{0}(\mathcal{M})$ satisfy $u \geqslant v$ iff $u(x) \geqslant v(x)$ for all $x \in \mathcal{M}$. The following lemmas summarize the main properties of order relations in Banach spaces.

Lemma 17. Let $X, X_{+}$be an ordered Banach space. Then, for all elements $u, v, w \in X$, hold: (i) $u \geqslant u$; (ii) If $u \geqslant v$ and $v \geqslant u$, then $u=v$; (iii) If $u \geqslant v$ and $v \geqslant w$, then $u \geqslant w$.

Proof. The property that $u-u=0 \in X_{+}$implies that $u \geqslant u$. If $u \geqslant v$ and $v \geqslant u$ then $u-v \in X_{+}$and $-(u-v) \in X_{+}$, therefore $u-v=0$. Finally, if $u \geqslant v$ and $v \geqslant w$, then $u-v \in X_{+}$and $v-w \in X_{+}$, which means that $u-w=(u-v)+(v-w) \in X_{+}$.

Furthermore, the order relation is compatible with the vector space structure and with the limits of sequences.

Lemma 18. Let $X, X_{+}$be an ordered Banach space. Then, for all $u, \hat{u}, v, \hat{v}, w \in X$, and $a, b \in \mathbb{R}$, the following hold:

(i) If $u \geqslant v$ and $a \geqslant b \geqslant 0$, then $a u \geqslant b v$;

(ii) If $u \geqslant v$ and $\hat{u} \geqslant \hat{v}$, then $u+\hat{u} \geqslant v+\hat{v}$;

(iii) If $u_{n} \geqslant v_{n}$ for all $n \in \mathbb{N}$, then $\lim _{n \rightarrow \infty} u_{n} \geqslant \lim _{n \rightarrow \infty} v_{n}$.

Proof. The first two properties are straightforward to prove, and we do not do it here. The third property holds because the order cone is a closed set. Indeed, $u_{n} \geqslant v_{n}$ means that $u_{n}-v_{n} \in X_{+}$for all $n \in \mathbb{N}$, and then $\lim _{n \rightarrow \infty}\left(u_{n}-v_{n}\right) \in X_{+}$because $X_{+}$is closed, then Property (iii) follows.

The remaining order relations have some other interesting properties.

Lemma 19. Let $X, X_{+}$be an ordered Banach space. Then, for all $u, v, w \in X$, and $a \in \mathbb{R}$, the following hold:: (i) If $u \gg v$ and $v \gg w$, then $u \gg w$; (ii) If $u \gg v$ and $v \geqslant w$, then $u \gg w$; (iii) If $u \geqslant v$ and $v \gg w$, then $u \gg w$; (iv) If $u \gg v$ and $a>0$, then $a u \gg a v$.

The proof of Lemma 19 is similar to the previous lemma, and is not reproduced here. Given an ordered Banach space $X, X_{+}$, and two elements $u \geqslant v$, introduce the intervals

$$
[v, u]:=\{w \in X: v \leqslant w \leqslant u\}, \quad(v, u):=\{w \in X: v \ll w \ll u\} .
$$



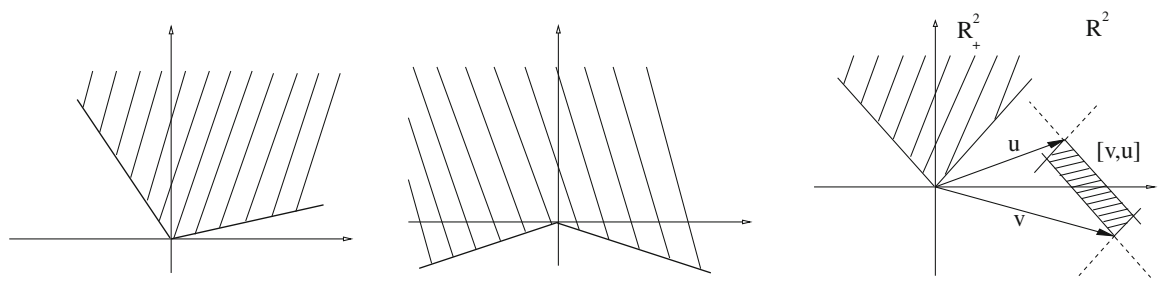

Fig. 3. The shaded regions in the first picture represent an order cone, while the second picture represents a cone that is not an order cone. The shaded region between $u$ and $v$ in the third picture represents the closed interval $[v, u]$, constructed with the order cone $\mathbb{R}_{+}^{2}$, which is also represented by a shaded region

Analogously, introduce the intervals $[v, u)$ and $(v, u]$. See Fig. 3 for an example in $X=\mathbb{R}^{2}$. Useful order cones for solving PDE are those that define an order structure in the Banach space which is related with the norm and the notion of boundedness. These types of order cones are called normal. More precisely, an order cone $X_{+}$in a Banach space $X$ is called a normal order cone iff there exists $0<a \in \mathbb{R}$ such that for all $u$, $v \in X$ with $0 \leqslant v \leqslant u$ holds $\|v\| \leqslant a\|u\|$.

Lemma 20. If $X, X_{+}$is an ordered Banach space with normal order cone $X_{+}$, then every closed interval in $X$ is bounded.

Proof. Let $w \in[v, u]$, then $v \leqslant w \leqslant u$, and so $0 \leqslant w-v \leqslant u-v$. Since the cone $X_{+}$ is normal, this implies that there exists $a>0$ such that $\|w-v\| \leqslant a\|u-v\|$. Then, the inequalities $\|w\| \leqslant\|w-v\|+\|v\| \leqslant a\|u-v\|+\|v\|$, which hold for all $w \in[v, u]$, establish the lemma.

Not every order cone is normal. For example, consider the Sobolev spaces $W^{k, p}$ of scalar-valued functions on an $n$-dimensional, closed manifold $\mathcal{M}$ (or a compact manifold with Lipschitz continuous boundary), where $k$ is a non-negative integer, and $p>1$ is a real number. An order cone in $W^{k, p}$ is defined translating the order on the real numbers, almost everywhere in $\mathcal{M}$, that is,

$$
W_{+}^{k, p}:=\left\{u \in W^{k, p}: u \geqslant 0 \text { a.e. in } \mathcal{M}\right\} .
$$

In the case $k=0$, that is, we have $W^{0, p}=L^{p}$, the order cone above is a normal cone $[2,54]$. However, in the case $k \geqslant 1$ the cone above cannot be normal, since on the one hand, the cone definition involves information only of the values of $u(x)$ and not of its derivatives; on the other hand, the norm in $W^{k, p}$ contains information of both the values of $u(x)$ and its derivatives. In the case of a compact manifold with boundary, since there are no boundary conditions on $\partial \mathcal{M}$ in the definition of $W^{k, p}$, there is no way to relate the values of a function in $\mathcal{M}$ with the values of its derivatives. (In other words, there is no Poincaré inequality for elements in $W^{k, p}$, with $k \geqslant 1$.)

An order cone $X_{+} \subset X$ is generating iff $\operatorname{Span}\left(X_{+}\right)=X$. An order cone $X_{+} \subset X$ is called total iff $\operatorname{Span}\left(X_{+}\right)$is dense in $X$. Total order cones are important because the order structure associated with them can be translated from the space $X$ into its dual space $X^{*}$.

Lemma 21. Let $X, X_{+}$be an ordered Banach space. If $X_{+}$is a total order cone, then an order cone in $X^{*}$ is given by the set $X_{+}^{*} \subset X^{*}$ defined as

$$
X_{+}^{*}:=\left\{u^{*} \in X^{*}: u^{*}(v) \geqslant 0 \quad \forall v \in X_{+}\right\} .
$$


Proof. We check the three properties in the definition of the order cone. The first property is satisfied because $X_{+}$is an order cone, so there exists $v \neq 0$ in $X_{+}$, and then there exists $u^{*} \neq 0$ in $X^{*}$ such that $u^{*}(v)=1 \geqslant 0$, so $X_{+}^{*}$ is non-empty. Trivially, $0 \in X_{+}^{*}$. Finally, $X_{+}^{*}$ is closed because the order relation $\geqslant$ for real numbers is used in its definition. The second property of an order cone is satisfied, because given any $u^{*}$, $v^{*} \in X_{+}^{*}$ and any non-negative $a, b \in \mathbb{R}$, then for all $\underline{u} \in X_{+}$,

$$
\left(a u^{*}+b v^{*}\right)(\underline{u})=a u^{*}(\underline{u})+b v^{*}(\underline{u}) \geqslant 0
$$

holds since each term is non-negative. This implies that $\left(a u^{*}+b v^{*}\right) \in X_{+}^{*}$. The third property is satisfied because the order cone $X_{+}$is total. Suppose that the element $u^{*} \in X_{+}^{*}$ and $-u^{*} \in X_{+}^{*}$, then for all $\underline{u} \in X_{+}$it holds that $u^{*}(\underline{u}) \geqslant 0$ and $-u^{*}(\underline{u}) \geqslant 0$, which implies that $u^{*}(\underline{u})=0$ for all $\underline{u} \in X_{+}$. Therefore, $u^{*} \in X_{+}^{\circ} \subset X^{*}$, where the superscript $\circ$ in $X_{+}^{\circ}$ means the Banach annihilator of the set $X_{+}$, which is a subset of the space $X^{*}$. Therefore, we conclude that $u^{*} \in\left[\operatorname{Span}\left(X_{+}\right)\right]^{\circ}$. Since the order cone is total, $\overline{\operatorname{Span}\left(X_{+}\right)}=X$, that implies $\left[\operatorname{Span}\left(X_{+}\right)\right]^{\circ}=\{0\}$, so $u^{*}=0$. This establishes the lemma.

An order cone $X_{+}$in a Banach space $X$ is called a solid cone iff $X_{+}$has non-empty interior. The following result asserts that solid order is generating. We remark that the converse is not true. In the examples below we present function spaces frequently used in solving PDE with order cones having empty interior which are indeed generating.

Lemma 22. Let $X, X_{+}$be an order Banach space. If $X_{+}$is a solid cone, then $X_{+}$is generating.

Proof. The cone $X_{+}$has a non-empty interior, so there exists $x_{0} \in \operatorname{int}\left(X_{+}\right)$and $x_{0} \neq 0$. This means that given any $x \in X$ there exists $0<a \in \mathbb{R}$ small enough such that both $x_{+}:=x_{0}+a x$ and $x_{-}:=x_{0}-a x$ belong to int $\left(X_{+}\right)$. But then, $x=\left(x_{+}-x_{-}\right) /(2 a)$, so $x \in \operatorname{Span}\left(X_{+}\right)$. This establishes the lemma.

Here is a list of examples of several order cones used in function spaces. All these examples use order cones obtained from the usual order in $\mathbb{R}$. In particular, they refer to scalar-valued functions on an $n$-dimensional, closed manifold $\mathcal{M}$ (or a compact manifold with Lipschitz boundary).

- Introduce on $C^{k}$ the cone $C_{+}^{k}:=\left\{u \in C^{k}: u(x) \geqslant 0 \quad \forall x \in \mathcal{M}\right\}$. This is an order cone for all non-negative integers $k$. The cone is a normal cone in the particular case $k=0$. The cone is solid for all $k \geqslant 0$, therefore it is a generating cone.

- Introduce on $L^{\infty}$ the cone $L_{+}^{\infty}:=\left\{u \in L^{\infty}: u \geqslant 0\right.$ a.e. in $\left.\mathcal{M}\right\}$. This is a normal, order cone. It is a solid cone, therefore it is generating.

- Introduce on $W^{k, \infty}$ the cone $W_{+}^{k, \infty}:=\left\{u \in W^{k, \infty}: u \geqslant 0\right.$ a.e. in $\left.\mathcal{M}\right\}$. This is an order cone. It is not normal for $k \geqslant 1$. The cone is solid, therefore it is generating.

- Introduce on $L^{p}$ the cone $L_{+}^{p}:=\left\{u \in L^{p}: u \geqslant 0\right.$ a.e. in $\left.\mathcal{M}\right\}$. This is a normal, order cone for every real number $p \geqslant 1$. The cone is not solid, however it is a generating cone.

- Introduce on $W^{k, p}$ the cone $W_{+}^{k, p}:=\left\{u \in W^{k, p}: u \geqslant 0\right.$ a.e. in $\left.\mathcal{M}\right\}$. This is an order cone for every real number $p \geqslant 1$. The cone is not normal for $k \geqslant 1$. The cone is not solid for $k p \leqslant n$, and it is solid for $k p>n$. In both cases, the cone is generating. 
A key concept that becomes possible in ordered Banach spaces is that of an operator satisfying a maximum principle. We have not seen in the literature an approach to maximum principles on ordered Banach spaces in the generality we now present. Let $X$, $X_{+}$and $Y, Y_{+}$be ordered Banach spaces. An operator $A: D_{A} \subset X \rightarrow Y$ satisfies the maximum principle iff for every $u, v \in D_{A}$ such that $A u-A v \in Y_{+}, u-v \in X_{+}$holds. In the particular case that the operator $A$ is linear, then it satisfies the maximum principle iff for all $u \in X$ such that $A u \in Y_{+}, u \in X_{+}$holds. The main example is the Laplace operator acting on scalar-valued functions defined on different domains. It is shown later on in this Appendix that the inverse of an operator that satisfies the maximum principle is monotone increasing. The following result gives a simple sufficient condition for an operator to satisfy the maximum principle. This result is useful on weak formulations of PDE.

Lemma 23. Let $X, X_{+}$be an ordered Banach space, and $A: X \rightarrow X^{*}$ be a linear and coercive map. Assume that $X_{+}$is a generating order cone, and that for all $u \in X$ such that $A u \in X_{+}^{*}$ there exists a decomposition $u=u^{+}-u^{-}$with $u^{+}, u^{-} \in X_{+}$that also satisfies $A u^{+}\left(u^{-}\right)=0$. Then, the operator A satisfies the maximum principle.

Proof. Since the order cone $X_{+}$is generating, the space $X^{*}$ is also an ordered Banach space. Denote its order cone by $X_{+}^{*}$. The assumption that the order cone $X_{+}$is generating also implies that for any element $u \in X$ there exists a decomposition $u=u^{+}-u^{-}$ with $u^{+}, u^{-} \in X_{+}$. By hypothesis, there exists at least one decomposition with the extra property that $A u^{+}\left(u^{-}\right)=0$. Now, by definition of the order in the space $X^{*}$ we have that

$$
A u \in X_{+}^{*} \Leftrightarrow A u(\underline{u}) \geqslant 0 \quad \forall \underline{u} \in X_{+} .
$$

Pick as a test function $\underline{u}=u^{-}$. Then,

$$
0 \leqslant A u\left(u^{-}\right)=A\left(u^{+}-u^{-}\right)\left(u^{-}\right)=A u^{+}\left(u^{-}\right)-A u^{-}\left(u^{-}\right)=-A u^{-}\left(u^{-}\right),
$$

where the last equality comes from the condition $A u^{+}\left(u^{-}\right)=0$. Therefore, we have

$$
A u^{-}\left(u^{-}\right) \leqslant 0 \Rightarrow u^{-}=0,
$$

because $A$ is coercive. So we showed that $u=u^{+} \in X_{+}$. This establishes the lemma.

An example is the weak form of the shifted Laplace-Beltrami operator $\Delta+s$ on scalar functions on a closed manifold $\mathcal{M}$, where $s>0$. Consider the case $X=W^{1,2}$, with $Y=X^{*}=W^{-1,2}$, and $X_{+}=W_{+}^{1,2}$, while $Y_{+}=W_{+}^{-1,2}$. The Laplace operator in this case is given by $A: X \rightarrow X^{*}$ with action $A u(v):=(\nabla u, \nabla v)$. It is not difficult to check that this operator satisfies the hypothesis in Lemma 23. Therefore, this operator satisfies the maximum principle, that is, $A u \in W_{+}^{-1,2}$ implies $u \in W_{+}^{1,2}$, that is, $u \geqslant 0$ a.e. in the manifold $\mathcal{M}$.

A.3. Monotone increasing maps. Let $X, X_{+}$and $Y, Y_{+}$be two ordered Banach spaces. An operator $F: X \rightarrow Y$ is monotone increasing iff for all $x, \underline{x} \in X$ such that $x-\underline{x} \in X_{+}$, $F(x)-F(\underline{x}) \in Y_{+}$holds. An operator $F: X \rightarrow Y$ is monotone decreasing iff for all $x, \underline{x} \in X$ such that $x-\underline{x} \in X_{+}$it holds that $-[F(x)-F(\underline{x})] \in Y_{+}$. The main result for these types of maps is the following; it can be found as Theorem 7.A in [54], p. 283, and Corollary 7.18 on p. 284 . We reproduce it here for completeness, without the proof. 
Theorem 10 (Fixed point for increasing operators). Let $X$ be an ordered Banach space, with a normal order cone $X_{+}$. Let $T:\left[x_{-}, x_{+}\right] \subset X \rightarrow X$ be a monotone increasing, compact map. If $-\left[x_{-}-T\left(x_{-}\right)\right] \in X_{+}$and $x_{+}-T\left(x_{+}\right) \in X_{+}$, then the iterations

$$
\begin{aligned}
x_{n+1}:=T\left(x_{n}\right), & x_{0}=x_{-}, \\
\hat{x}_{n+1}:=T\left(\hat{x}_{n}\right), & \hat{x}_{0}=x_{+},
\end{aligned}
$$

converge to $x$ and $\hat{x} \in\left[x_{-}, x_{+}\right]$, respectively, and the following estimate holds:

$$
x_{-} \leqslant x_{n} \leqslant x \leqslant \hat{x} \leqslant \hat{x}_{n} \leqslant x_{+}, \quad \forall n=\mathbb{N} .
$$

We are interested in the following class of nonlinear problems: Find an element $x \in X$ which solves the equation

$$
A x+F(x)=0,
$$

where the principal part involves an invertible linear operator $A: X \rightarrow Y$ satisfying the maximum principle, and the non-principal part involves a nonlinear operator $F: X \rightarrow Y$ which has monotonicity properties. We now establish some basic results for this class of problems. The first two results relate linear, invertible operators that satisfy the maximum principle with monotone increasing (decreasing) operators.

Lemma 24. Let $X, X_{+}$and $Y, Y_{+}$be two ordered Banach spaces. Let $A: X \rightarrow Y$ be a linear, invertible operator satisfying the maximum principle. Then, the inverse operator $A^{-1}: Y \rightarrow X$ is monotone increasing.

Proof. Let $y, \underline{y} \in Y$ be such that $y-\underline{y} \in Y_{+}$. Then,

$$
A\left(A^{-1}(y-\underline{y})\right) \in Y_{+} \quad \Rightarrow \quad A^{-1}(y-\underline{y}) \in X_{+} \quad \Leftrightarrow \quad A^{-1} y-A^{-1} \underline{y} \in X_{+} .
$$

This establishes that the operator $A^{-1}$ is monotone increasing.

Lemma 25. Let $X, X_{+}$and $Y, Y_{+}$be two ordered Banach spaces. Let $A: X \rightarrow Y$ be a linear, invertible operator satisfying the maximum principle. Let $F: X \rightarrow Y$ be a monotone decreasing (increasing) operator. Then, the operator $T: X \rightarrow X$ given by $T:=-A^{-1} F$ is monotone increasing (decreasing).

Proof. Assume first that the operator $F$ is monotone decreasing. So, given any $x, \underline{x} \in X$ such that $x-\underline{x} \in X_{+}$, the following inequalities hold:

$$
\begin{aligned}
x-\underline{x} \in X_{+} & \Rightarrow-[F(x)-F(\underline{x})] \in Y_{+}, \\
& \Leftrightarrow A\left(-A^{-1}[F(x)-F(\underline{x})]\right) \in Y_{+}, \\
& \Rightarrow-A^{-1}[F(x)-F(\underline{x})] \in X_{+}, \\
& \Leftrightarrow-\left[A^{-1} F(x)-A^{-1} F(\underline{x})\right] \in X_{+}, \\
& \Leftrightarrow T(x)-T(\underline{x}) \in X_{+},
\end{aligned}
$$

which establishes that the operator $T$ is monotone increasing. In the case that the operator $F$ is monotone increasing, then the first line in the proof above changed into $x-\underline{x} \in X_{+}$ implies that $F(x)-F(\underline{x}) \in Y_{+}$, and then all the remaining inequalities in the proof above are reverted. This establishes the lemma. 
The next result translates the inequalities that satisfy sub- and super-solutions to the equation $A x+F(x)=0$, into inequalities for the operator $T=-A^{-1} F$.

Lemma 26. Assume the hypothesis in Lemma 25.

If there exists an element $x_{+} \in X$ such that $A x_{+}+F\left(x_{+}\right) \in Y_{+}$, then this element satisfies that $x_{+}-T\left(x_{+}\right) \in X_{+}$.

If there exists an element $x_{-} \in X$ such that $-\left[A x_{-}+F\left(x_{-}\right)\right] \in Y_{+}$, then this element satisfies that $-\left[x_{-}-T\left(x_{-}\right)\right] \in X_{+}$.

Proof. The first statement in the lemma can be shown as follows:

$$
\begin{aligned}
A x_{+}+F\left(x_{+}\right) \in Y_{+} & \Leftrightarrow A\left(x_{+}+A^{-1} F\left(x_{+}\right)\right) \in Y_{+} \\
& \Rightarrow x_{+}+A^{-1} F\left(x_{+}\right) \in X_{+},
\end{aligned}
$$

which then establishes that $x_{+}-T\left(x_{+}\right) \in X_{+}$. In a similar way, the second statement in the lemma can be shown as follows:

$$
\begin{aligned}
-\left[A x_{-}+F\left(x_{-}\right)\right] \in Y_{+} & \Leftrightarrow A\left(-x_{-}-A^{-1} F\left(x_{-}\right)\right) \in Y_{+} \\
& \Rightarrow-x_{-}-A^{-1} F\left(x_{-}\right) \in X_{+},
\end{aligned}
$$

which then establishes that $-\left[x_{-}-T\left(x_{-}\right)\right] \in X_{+}$. This establishes the lemma.

For nonlinear problems of the form (A.2), one can use Theorem 10 for monotone nonlinearities to conclude the following.

Corollary 2. (Semi-linear equations with sub-/super-solutions) Let $X, X_{+}$and $Y, Y_{+}$be two ordered Banach spaces where $X_{+}$is a normal order cone. Let $A: X \rightarrow Y$ be a linear, invertible operator satisfying the maximum principle. Let $x_{+}, x_{-} \in X$ be elements such that $\left(x_{+}-x_{-}\right) \in X_{+}$, and then assume that the operator $F:\left[x_{-}, x_{+}\right] \subset X \rightarrow Y$ is monotone decreasing and compact. If the elements $x_{-}$and $x_{+}$satisfy the relations

$$
-\left[A x_{-}+F\left(x_{-}\right)\right] \in Y_{+}, \quad A x_{+}+F\left(x_{+}\right) \in Y_{+},
$$

then there exists a solution $x \in\left[x_{-}, x_{+}\right] \subset X$ of the equation $A x+F(x)=0$.

Proof. The operator $A$ is invertible, then rewrite the equation $A x+F(x)=0$ as a fixed-point equation,

$$
x=-A^{-1} F(x)=: T(x) .
$$

By Lemma 25, we know that the map $T: X \rightarrow X$ is monotone increasing. Moreover, this operator $T$ is compact, since it is the composition of the continuous mapping $-A^{-1}$ and the compact map $F$. The elements $x_{-}$and $x_{+}$satisfy Eq. (A.3), therefore, by Lemma 26, they are also sub- and super-solutions for the fixed-point equation involving the map $T$. It follows from Theorem 10 that there exists an element $x \in X$ solution to the fixed-point equation (A.4), and this solution satisfies the bounds $x_{-} \leqslant x \leqslant x_{+}$. 
A.4. Sobolev spaces on closed manifolds. In this Appendix we will recall some properties of Sobolev spaces of sections of vector bundles over closed manifolds. The following definition makes precise what we mean by fractional order Sobolev spaces. We expect that without much difficulty all the results in this paper can be modified to reflect other smoothness classes such as Bessel potential spaces or general Besov spaces.

Definition 2. For $s \geqslant 0$ and $1 \leqslant p \leqslant \infty$, we denote by $W^{s, p}\left(\mathbb{R}^{n}\right)$ the space of all distributions $u$ defined in $\mathbb{R}^{n}$, such that

(a) when $s=m$ is an integer,

$$
\|u\|_{m, p}=\sum_{|v| \leqslant m}\left\|\partial^{v} u\right\|_{p}<\infty
$$

where $\|\cdot\|_{p}$ is the standard $L^{p}$-norm in $\mathbb{R}^{n}$;

(b) and when $s=m+\sigma$ with $m$ (nonnegative) integer and $\sigma \in(0,1)$,

$$
\|u\|_{s, p}=\|u\|_{m, p}+\sum_{|\nu|=m}\left\|\partial^{v} u\right\|_{\sigma, p}<\infty,
$$

where

$$
\|u\|_{\sigma, p}=\left(\iint_{\mathbb{R}^{n} \times \mathbb{R}^{n}} \frac{|u(x)-u(y)|^{p}}{|x-y|^{n+\sigma p}} d x d y\right)^{\frac{1}{p}}, \quad \text { for } 1 \leqslant p<\infty,
$$

and

$$
\|u\|_{\sigma, \infty}=\operatorname{ess}_{\sup }, y \in \mathbb{R}^{n} \frac{|u(x)-u(y)|}{|x-y|^{\sigma}} .
$$

For $s<0$ and $1<p<\infty, W^{s, p}\left(\mathbb{R}^{n}\right)$ denotes the topological dual of $W^{-s, p^{\prime}}\left(\mathbb{R}^{n}\right)$, where $\frac{1}{p}+\frac{1}{p^{\prime}}=1$.

These well known spaces are Banach spaces with corresponding norms, and become Hilbert spaces when $p=2$. We refer to $[18,46]$ and references therein for further properties.

Now we will define analogous spaces on closed manifolds. Let $\mathcal{M}$ be an $n$-dimensional smooth closed manifold, and let $\left\{\left(U_{i}, \varphi_{i}\right)\right\}$ be a collection of charts such that $\left\{U_{i}\right\}$ forms a finite cover of $\mathcal{M}$. Then for any distribution $u \in C_{0}^{\infty}\left(U_{i}\right)^{*}$, the pull-back $\varphi_{i}^{*}(u) \in C_{0}^{\infty}\left(\varphi_{i}\left(U_{i}\right)\right)^{*}$ is defined by $\varphi_{i}^{*}(u)(v)=u\left(v \circ \varphi_{i}\right)$ for all $v \in C_{0}^{\infty}\left(\varphi_{i}\left(U_{i}\right)\right)$. Extending $\varphi_{i}^{*}(u)$ by zero outside $\varphi_{i}\left(U_{i}\right)$, in the following we treat it as a distribution on $\mathbb{R}^{n}$. Let $\left\{\chi_{i}\right\}$ be a smooth partition of unity subordinate to $\left\{U_{i}\right\}$.

Definition 3. For $s \in \mathbb{R}$ and $p \in(1, \infty)$, we denote by $W^{s, p}(\mathcal{M})$ the space of all distributions $u$ defined in $\mathcal{M}$, such that

$$
\|u\|_{s, p}=\sum_{i}\left\|\varphi_{i}^{*}\left(\chi_{i} u\right)\right\|_{s, p}<\infty
$$

where the norm under the sum is the $W^{s, p}\left(\mathbb{R}^{n}\right)$-norm. In case $s \geqslant 0$, these Sobolev spaces can also be defined for $p=1$ and $p=\infty$. 
We collect the most basic properties of these spaces in the following lemma. Recall that a Riemannian metric on $\mathcal{M}$ induces a volume form on $\mathcal{M}$, so that $L^{p}$ spaces can be defined on $\mathcal{M}$ (cf. [43]).

Lemma 27. Either let $s \geqslant 0$ and $p \in[1, \infty]$ or let $s<0$ and $p \in(1, \infty)$. Then the space $W^{s, p}(\mathcal{M})$ is a Banach space. It is independent of the choice of the covering charts $\left\{\left(U_{i}, \varphi_{i}\right)\right\}$ and the partition of unity $\left\{\chi_{i}\right\}$. In particular, the different norms (A.5) are equivalent. Moreover, the following are true when $\mathcal{M}$ is equipped with a smooth Riemannian metric.

(a) Let $\nabla$ be the Levi-Civita connection associated to the Riemannian metric. Then for any nonnegative integer $m$,

$$
\|u\|_{m, p}^{\prime}=\sum_{i=0}^{m}\left\|\nabla^{i} u\right\|_{p}
$$

is an equivalent norm on $W^{m, p}(\mathcal{M})$. In particular, we have $W^{0, p}(\mathcal{M})=L^{p}(\mathcal{M})$.

(b) Identifying $C^{\infty}(\mathcal{M})$ as a subspace of distributions via the $L^{2}$-inner product, $C^{\infty}(\mathcal{M})$ is densely embedded in $W^{s, p}(\mathcal{M})$ for any $s \in \mathbb{R}$ and $p \in(1, \infty)$.

(c) Let $s \in \mathbb{R}$ and $p \in(1, \infty)$. Then the $L^{2}$-inner product on $C^{\infty}(\mathcal{M})$ extends uniquely to a continuous bilinear pairing $W^{s, p}(\mathcal{M}) \otimes W^{-s, p^{\prime}}(\mathcal{M}) \rightarrow \mathbb{R}$, where $\frac{1}{p}+\frac{1}{p^{\prime}}=1$. Moreover, the pairing induces a topological isomorphism between $W^{-s, p^{\prime}}(\mathcal{M})$ and the topological dual space of $W^{s, p}(\mathcal{M})$.

Proof. See for example [3,19,43,45].

A main goal of this subsection is to extend the previous lemma to the case when the Riemannian metric is not smooth. The following result will be of importance.

Lemma 28. Let $s_{i} \geqslant s$ with $s_{1}+s_{2} \geqslant 0$, and $1 \leqslant p, p_{i} \leqslant \infty(i=1,2)$ be real numbers satisfying

$$
s_{i}-s \geqslant n\left(\frac{1}{p_{i}}-\frac{1}{p}\right), \quad s_{1}+s_{2}-s>n\left(\frac{1}{p_{1}}+\frac{1}{p_{2}}-\frac{1}{p}\right),
$$

where the strictness of the inequalities can be interchanged if $s \in \mathbb{N}_{0}$. In case $\min \left(s_{1}, s_{2}\right)<0$, in addition let $1<p, p_{i}<\infty$, and let

$$
s_{1}+s_{2} \geqslant n\left(\frac{1}{p_{1}}+\frac{1}{p_{2}}-1\right) .
$$

Then, the pointwise multiplication of functions extends uniquely to a continuous bilinear map

$$
W^{s_{1}, p_{1}}(\mathcal{M}) \otimes W^{s_{2}, p_{2}}(\mathcal{M}) \rightarrow W^{s, p}(\mathcal{M})
$$

Proof. A proof is given in [55] for the case $s \geqslant 0$, and by using a duality argument one can easily extend the proof to negative values of $s$.

Some important special cases are considered in the following corollary: 
Corollary 3. (a) If $p \in(1, \infty)$ and $s \in\left(\frac{n}{p}, \infty\right)$, then $W^{s, p}$ is a Banach algebra. Moreover, if in addition $q \in(1, \infty)$ and $\sigma \in[-s, s]$ satisfy $\sigma-\frac{n}{q} \in\left[-n-s+\frac{n}{p}, s-\frac{n}{p}\right]$, then the pointwise multiplication is bounded as a map $W^{s, p} \otimes W^{\sigma, q} \rightarrow W^{\sigma, q}$.

(b) Let $1<p, q<\infty$ and $\sigma \leqslant s \geqslant 0$ satisfy $\sigma-\frac{n}{q}<2\left(s-\frac{n}{p}\right)$ and $\sigma-\frac{n}{q} \leqslant s-\frac{n}{p}$. Then the pointwise multiplication is bounded as a map $W^{s, p} \otimes W^{s, p} \rightarrow W^{\sigma, q}$.

The following lemma is proved in [33] for the case $p=q=2$. With the help of Lemma 28, the proof can easily be adapted to the following general case.

Lemma 29. Let $p \in(1, \infty)$ and $s \in\left(\frac{n}{p}, \infty\right)$, and let $u \in W^{s, p}$. Let $\sigma \in[-1,1]$ and $\frac{1}{q} \in\left(\frac{1+\sigma}{2} \delta, 1-\frac{1-\sigma}{2} \delta\right)$, and let $v \in W^{\sigma, q}$, where $\delta=\frac{1}{p}-\frac{s-1}{n}$. Moreover, let $f:[\inf u, \sup u] \rightarrow \mathbb{R}$ be a smooth function. Then, we have

$$
\|v(f \circ u)\|_{\sigma, q} \leqslant C\|v\|_{\sigma, q}\left(\|f \circ u\|_{\infty}+\left\|f^{\prime} \circ u\right\|_{\infty}\|u\|_{s, p}\right),
$$

where the constant $C$ does not depend on $u, v$ or $f$.

Proof. We consider the case $\sigma=1$ first. Choosing a smooth Riemannian metric on $\mathcal{M}$, we have

$$
\begin{aligned}
\|v(f \circ u)\|_{1, q} & \leqslant C\left(\|v(f \circ u)\|_{q}+\|\nabla[v(f \circ u)]\|_{q}\right) \\
& \leqslant C\left(\|v(f \circ u)\|_{q}+\|(\nabla v)(f \circ u)\|_{q}+\left\|v\left(f^{\prime} \circ u\right) \nabla u\right\|_{q}\right) \\
& \leqslant C\left(\|v\|_{q}\|f \circ u\|_{\infty}+\|v\|_{1, q}\|f \circ u\|_{\infty}+\left\|f^{\prime} \circ u\right\|_{\infty}\|v \nabla u\|_{q}\right) .
\end{aligned}
$$

By Lemma 28 , for $\frac{1}{q} \geqslant \delta$, the last term can be bounded as

$$
\|v \nabla u\|_{q} \leqslant C\|v\|_{1, q}\|\nabla u\|_{s-1, p} \leqslant C\|v\|_{1, q}\|u\|_{s, p},
$$

proving the lemma for the case $\sigma=1$. By using duality one proves the case $\sigma=-1$ and $\frac{1}{q} \leqslant 1-\delta$, and the lemma follows from interpolation.

Let $\mathcal{M}$ be an $n$-dimensional smooth closed manifold, and let $E \rightarrow \mathcal{M}$ be a smooth vector bundle over $\mathcal{M}$. Analogously to Definition 3, we define the Sobolev space $W^{s, p}(E)$ of sections of $E$ by utilizing a finite trivializing cover of coordinate charts, a partition of unity subordinate to the cover, and the space $\left[W^{s, p}\left(\mathbb{R}^{n}\right)\right]^{k}$ of vector functions, where $k$ is the fiber dimension of $E$. Then, Lemma 27 holds for these spaces with obvious modifications. When there is no risk of confusion, we will omit the explicit specification of the vector bundle $E$ from the notation $W^{s, p}(E)$.

In the following lemma we consider nonsmooth Riemannian structures on $E$ and nonsmooth volume forms on $\mathcal{M}$.

Lemma 30. Let $\gamma \in(1, \infty)$ and $\alpha \in\left(\frac{n}{\gamma}, \infty\right)$. Fix on $\mathcal{M}$ a volume form of class $W^{\alpha, \gamma}$, and on $E$ a Riemannian structure of class $W^{\alpha, \gamma}$.

(a) Let $p \in(1, \infty)$ and $s \leqslant \min \left\{\alpha, \alpha+n\left(\frac{1}{p}-\frac{1}{\gamma}\right)\right\}$. Then identifying the space $C^{\infty}(E)$ of smooth sections of $E$ as a subspace of distributions via the $L^{2}$-inner product, $C^{\infty}(E)$ is densely embedded in $W^{s, p}(E)$.

(b) Let $s \in[-\alpha, \alpha], p \in(1, \infty)$, and $s-\frac{n}{p} \in\left[-n-\alpha+\frac{n}{\gamma}, \alpha-\frac{n}{\gamma}\right]$. Then the $L^{2}$-inner product on $C^{\infty}(E)$ extends uniquely to a continuous bilinear pairing $W^{s, p}(E) \otimes W^{-s, p^{\prime}}(E) \rightarrow \mathbb{R}$, where $\frac{1}{p}+\frac{1}{p^{\prime}}=1$. Moreover, the pairing induces $a$ topological isomorphism $\left[W^{s, p}(E)\right]^{*} \cong W^{-s, p^{\prime}}(E)$. 
Proof. We will prove the lemma for scalar functions on $\mathcal{M}$, i.e., for the trivial bundle $E=\mathcal{M} \times \mathbb{R}$. The general case is only more technical.

Fixing a smooth volume form on $\mathcal{M}$ and denoting the associated $L^{2}$-inner product by $(\cdot, \cdot)_{*}$, the $L^{2}$-inner product associated to the nonsmooth volume form (and the nonsmooth metric on $\mathcal{M} \times \mathbb{R}$ ) satisfies

$$
(u, v)_{L^{2}}=(h u, v)_{*}, \quad u, v \in C^{\infty}(\mathcal{M}),
$$

with some strictly positive function $h \in W^{\alpha, \gamma}$. From Lemma 28, we have that multiplication by $h$ is continuous on $W^{s, p}$ for $s \in[-\alpha, \alpha], p \in(1, \infty)$, and $s-\frac{n}{p} \in$ $\left[-n-\alpha+\frac{n}{\gamma}, \alpha-\frac{n}{\gamma}\right]$. Since $h>0$ this operation is invertible hence a homeomorphism on $W^{s, p}$. Now by using Lemma 27 we complete the proof.

Corollary 4. Let $\gamma \in(1, \infty)$ and $\alpha \in\left(\frac{n}{\gamma}, \infty\right)$. Fix on $\mathcal{M}$ a volume form of class $W^{\alpha, \gamma}$, and on $E$ a Riemannian structure of class $W^{\alpha, \gamma}$. With $s \in[-\alpha, \alpha], p \in(1, \infty)$, and $s-\frac{n}{p} \in\left[-n-\alpha+\frac{n}{\gamma}, \alpha-\frac{n}{\gamma}\right]$, let $A: L^{p} \rightarrow W^{s, p}$ be a bounded linear operator and let $A^{*}$ be its formal $L^{2}$-adjoint, i.e., let

$$
(A u, v)_{L^{2}}=\left(u, A^{*} v\right)_{L^{2}}, \quad \text { for } u, v \in C^{\infty}(E) .
$$

Then, $A^{*}$ extends uniquely to a bounded linear map $A^{*}: W^{-s, p^{\prime}} \rightarrow L^{p^{\prime}}$, and we have

$$
\langle A u, v\rangle=\left\langle u, A^{*} v\right\rangle, \quad \text { for } u \in L^{p}(E), \quad v \in W^{-s, p^{\prime}}(E),
$$

where $\langle\cdot, \cdot\rangle$ denotes the extension of the $L^{2}$-inner product.

Proof. This is an application of Lemma 30.

A.5. Elliptic operators on closed manifolds. In this Appendix we will state a priori estimates for general elliptic operators in some Sobolev spaces. Let $\mathcal{M}$ be an $n$-dimensional smooth closed manifold, and let $E \rightarrow \mathcal{M}$ be a smooth vector bundle over $\mathcal{M}$.

Let $C^{-\infty}(E)$ be the topological dual of the space $C^{\infty}(E)$ of smooth sections of $E$. Then for $m \in \mathbb{N}, \alpha \in \mathbb{R}$, and $\gamma \in[1, \infty]$, we define $\mathcal{D}_{m}^{\alpha, \gamma}(E)$ to be the space of differential operators $A: C^{\infty}(E) \rightarrow C^{-\infty}(E)$ that can be written in local coordinates (trivializing $E$ ) as

$$
A=\sum_{|v| \leqslant m} a^{v} \partial_{\nu} \quad \text { with } a^{\nu} \in W^{\alpha-m+|v|, \gamma}\left(\mathbb{R}^{n}, \mathbb{R}^{k \times k}\right), \quad|v| \leqslant m,
$$

where $k$ is the fiber dimension of $E$.

One can easily verify that if the metric of a Riemannian manifold is in $W^{\alpha, \gamma}$ with $\alpha \gamma>n$, then both the Laplace-Beltrami operator and vector Laplacian defined in (2.17) are in the classes $\mathcal{D}_{2}^{\alpha, \gamma}(\mathcal{M} \times \mathbb{R})$ and $\mathcal{D}_{2}^{\alpha, \gamma}(T \mathcal{M})$, respectively.

Lemma 31. Let $A$ be a differential operator of class $\mathcal{D}_{m}^{\alpha, \gamma}(E)$. Then, A can be extended to a bounded linear map

$$
A: W^{s, q}(E) \rightarrow W^{\sigma, q}(E)
$$


for $q \in(1, \infty), s \geqslant m-\alpha$, and $\sigma$ satisfying

$$
\begin{gathered}
\sigma \leqslant \min \{s, \alpha\}-m, \quad \sigma<s-m+\alpha-\frac{n}{\gamma}, \\
\sigma-\frac{n}{q} \leqslant \alpha-\frac{n}{\gamma}-m, \quad \text { and } s-\frac{n}{q} \geqslant m-n-\alpha+\frac{n}{\gamma} .
\end{gathered}
$$

Proof. This is a straightforward application of Lemma 28.

The Laplace-Beltrami operator and vector Laplacian are elliptic operators. We now consider local a priori estimates for general elliptic operators. For any subset $U \subset \mathcal{M}$, the $W^{s, p}(U)$-norm is denoted by $\|\cdot\|_{s, p, U}$.

Lemma 32. Let $A \in \mathcal{D}_{m}^{\alpha, \gamma}(E)$ be an elliptic operator with $\alpha-\frac{n}{\gamma}>\max \left\{0, \frac{m-n}{2}\right\}$. Let $q \in(1, \infty), s \in(m-\alpha, \alpha]$, and $s-\frac{n}{q} \in\left(m-n-\alpha+\frac{n}{\gamma}, \alpha-\frac{n}{\gamma}\right]$. Then for any $y \in \mathcal{M}$, there exists a constant $c>0$ and open neighborhoods $K \subset U \subset \mathcal{M}$ of y such that

$$
c\|\chi u\|_{s, q} \leqslant\|A u\|_{s-m, q}+\|u\|_{s-1, q, U}
$$

for any $u \in W^{s, q}(E)$ and $\chi \in C_{0}^{\infty}(K)$ with $\chi \geqslant 0$.

Proof. We work in a local chart containing $y$, which trivializes $E$. Let $K$ be the open ball of radius $r$ centered at $y$ contained in the domain of the chart and extend the coefficients of $A$ outside $K$ so that the resulting operator is still in $\mathcal{D}_{m}^{\nu, \gamma}$, with appropriate vector fields over $\mathbb{R}^{n}$. We make the decomposition $A=L+R+B$, where $L$ is the highest order term of $A$ with coefficients frozen at $y$, and $R$ is what remains in the highest order terms, i.e.,

$$
L=\sum_{|v|=m} a^{\nu}(y) \partial_{\nu}, \quad R=\sum_{|\nu|=m}\left[a^{\nu}-a^{\nu}(y)\right] \partial_{\nu} .
$$

Obviously $B=A-L-R$ are the lower order terms. Let $u \in W^{s, q}$ with supp $u \subset K$. From the theory of constant coefficient elliptic operators, we infer the existence of a constant $c>0$ such that for any $u \in W^{s, q}(E)$ with supp $u \subset K$,

$$
\begin{aligned}
c\|u\|_{s, q} & \leqslant\|L u\|_{s-m, q}+\|u\|_{s-m, q} \\
& \leqslant\|A u\|_{s-m, q}+\|R u\|_{s-m, q}+\|B u\|_{s-m, q}+\|u\|_{s-m, q} .
\end{aligned}
$$

Since $\alpha>\frac{n}{\gamma}$, without loss of generality we can assume for $|\nu|=m$ that $a^{\nu} \in C^{0, h}$ for some $h>0$, so

$$
\|R u\|_{s-m, q} \leqslant C r^{h}\|u\|_{s, q},
$$

where $C$ is a constant depending only on $A$. By choosing $r$ so small that $C r^{h} \leqslant \frac{c}{2}$, we have

$$
\frac{c}{2}\|u\|_{s, q} \leqslant\|A u\|_{s-m, q}+\|B u\|_{s-m, q}+\|u\|_{s-m, q} .
$$

Now we will work with the lower order term. Choose $\delta \in\left(0, \alpha-\frac{n}{\gamma}\right)$ such that $\delta \leqslant \min \left\{1, s+\alpha-m, s-\frac{n}{q}+\alpha-\frac{n}{\gamma}+n-m\right\}$. We have $B \in \mathcal{D}_{m-1}^{\alpha-1, \gamma}$, so by Lemma 31 , 
$B: W^{s-\delta, \gamma} \rightarrow W^{s-m, \gamma}$ is bounded. Then using a well known interpolation inequality, we get

$$
\|B u\|_{s-m, q} \leqslant C\|u\|_{s-\delta, q} \leqslant C \varepsilon\|u\|_{s, q}+C^{\prime} \varepsilon^{-(m-\delta) / \delta}\|u\|_{s-m, q},
$$

for any $\varepsilon>0$. Choosing $\varepsilon>0$ sufficiently small, we conclude that

$$
c\|u\|_{s, q} \leqslant\|A u\|_{s-m, q}+\|u\|_{s-m, q}, \quad \forall u \in W^{s, q}(E), \quad \operatorname{supp} u \subset K .
$$

We apply this inequality to $\chi u$, and then observing that $[A, \chi]$ is in $\mathcal{D}_{m-1}^{\alpha, \gamma}(\mathcal{M})$, we obtain (A.6).

We can easily globalize the above result as follows:

Corollary 5. Let the conditions of Lemma 32 hold. Then there exists a constant $c>0$ such that

$$
c\|u\|_{s, q} \leqslant\|A u\|_{s-m, q}+\|u\|_{s-m, q}, \quad \forall u \in W^{s, q}(E) .
$$

Proof. We first cover $\mathcal{M}$ by open neighborhoods $K$ by applying Lemma 32 to every point $y \in \mathcal{M}$, and then choose a finite subcover of the resulting cover. Then a partition of unity argument gives (A.7) with the term $\|u\|_{s-m, q}$ replaced by $\|u\|_{s-1, q}$, and finally one can use an interpolation inequality to get the conclusion.

Let us recall the following well known results from functional analysis.

Lemma 33. Let $X$ and $Y$ be Banach spaces with continuous embedding $X \hookrightarrow Y$. Let $A: X \rightarrow Y$ be a continuous linear map. Then

(a) A necessary and sufficient condition that the graph of A be closed in $X \times Y$ is that there exists a constant $c>0$ such that $c\|u\|_{X} \leqslant\|A u\|_{Y}+\|u\|_{Y}$ for all $u \in X$.

(b) If in addition the embedding $X \hookrightarrow Y$ is compact then the range of $A$ is closed and the kernel of $A$ is finite-dimensional.

As an immediate consequence, we obtain the following result.

Lemma 34. Let $A \in \mathcal{D}_{m}^{\alpha, \gamma}(E)$ be an elliptic operator with $\alpha-\frac{n}{\gamma}>\max \left\{0, \frac{m-n}{2}\right\}$. Let $q \in(1, \infty), s \in(m-\alpha, \alpha]$, and $s-\frac{n}{q} \in\left(m-n-\alpha+\frac{n}{\gamma}, \alpha-\frac{n}{\gamma}\right]$. Then, the operator $A: W^{s, q}(E) \rightarrow W^{s-m, q}(E)$ is semi-Fredholm, i.e., its range is closed and the kernel is finite-dimensional.

A.6. Maximum principles on closed manifolds. In this Appendix, we present maximum principles for the operators of the form $-\nabla \cdot(u \nabla)$ with positive function $u$, followed by a simple application. These types of results are well known, but nevertheless we state them here for completeness.

It is convenient at times when working with barriers and maximum principle arguments to split real valued functions into positive and negative parts; we will use the following notation for these concepts:

$$
\phi^{+}:=\max \{\phi, 0\}, \quad \phi^{-}:=-\min \{\phi, 0\},
$$

whenever they make sense. In the proof of the following lemma we will use the fact that for $\phi \in W^{1, p}, \phi^{+} \in W^{1, p}$ holds, and so $\phi^{-} \in W^{1, p}$, cf. [38]. 
Lemma 35. Let $p \in(1, \infty)$ and $s \in\left(\frac{n}{p}, \infty\right) \cap[1, \infty)$, and let $\left(\mathcal{M}, h_{a b}\right)$ be an $n$-dimensional, smooth, closed manifold with a Riemannian metric $h_{a b} \in W^{s, p}$. Moreover, let $u \in W^{s, p}$ be a function with $u>0$ and let $f \in W^{s-2, p}$. Let $\phi \in W^{s, p}$ be such that

$$
\langle u \nabla \phi, \nabla \varphi\rangle+\langle f, \phi \varphi\rangle \geqslant 0, \quad \text { for all } \varphi \in C_{+}^{\infty} .
$$

(a) If $f \neq 0$ and $\langle f, \varphi\rangle \geqslant 0$ for all $\varphi \in C_{+}^{\infty}$, then $\phi \geqslant 0$.

(b) If $\mathcal{M}$ is connected and $\phi \geqslant 0$, then either $\phi \equiv 0$ or $\phi>0$ everywhere.

Proof. For (a), we will follow the proof of [33, Lemma 2.9]. Since $\phi \in W^{1, n}$, we have $\phi^{-} \in W_{+}^{1, n}$ and $-\phi \phi^{-} \in W_{+}^{1, n}$. Note that $W^{1, n} \hookrightarrow\left(W^{s-2, p}\right)^{*}$ by $n \geqslant 2$. Now, using the positivity of $f$ and the property (A.8), by density we get

$$
0 \geqslant\left\langle f, \phi \phi^{-}\right\rangle \geqslant-\left\langle u \nabla \phi, \nabla \phi^{-}\right\rangle=\left\langle u \nabla \phi^{-}, \nabla \phi^{-}\right\rangle,
$$

implying that $\phi^{-}=$const. So if $\phi<0$, it would have to be a negative constant. But property (A.8) gives $\langle f, \varphi\rangle \leqslant 0$ for all $\varphi \in C_{+}^{\infty}$, which, in combination with the positivity, implies $f=0$. This contradicts the hypothesis $f \neq 0$ and proves (a).

Now we will prove (b). Since $\phi$ is continuous, the level set $\phi^{-1}(0) \subset \mathcal{M}$ is closed. Following the proof of [35, Lemma 5.3], we apply the weak Harnack inequality [47, Theorem 5.2] to show that $\phi^{-1}(0)$ is also open. Then by connectedness of $\mathcal{M}$ we will have the proof.

The weak Harnack inequality [47, Theorem 5.2] can be applied to second order elliptic operators of the form

$$
L \phi=\partial_{i}\left(a^{i j} \partial_{j} \phi+a^{i} \phi\right)+b^{j} \partial_{j} \phi+a \phi,
$$

where $a^{i j}$ are continuous, and $a^{i}, b^{j} \in L^{2 t}$, and $a \in L^{t}$ for some $t>\frac{n}{2}$. The first term in (A.8) satisfies these conditions, and the second term can be cast into a form satisfying the conditions (details can be found in the proof of [35, Lemma 5.3]). Now suppose that $\phi(x)=0$ for some $x \in \mathcal{M}$, and let us work in local coordinates around $x$. Then the weak Harnack inequality says that for sufficiently small $R>0$, and for some $p>t^{\prime}$,

$$
\|\phi\|_{L^{p}(B(x, 2 R))} \leqslant C R^{\frac{n}{p}} \inf _{B(x, R)} \phi,
$$

where $B(x, R)$ denotes the open ball of radius $R$ (in the background flat metric) centered at $x$, and $C$ is a constant that depends only on $t, p$, and the differential operator. Since $\phi(x)=0$ and $\phi$ is nonnegative, the infimum is zero and the inequality implies that $\phi \equiv 0$ in a neighborhood of $x$. Hence the set $\phi^{-1}(0)$ is open.

Lemma 36. Let the hypotheses of Lemma 35 (b) hold, and define the operator $L$ : $W^{s, p} \rightarrow W^{s-2, p}$ by

$$
\langle L \phi, \varphi\rangle=\langle u \nabla \phi, \nabla \varphi\rangle+\langle f, \phi \varphi\rangle, \quad \phi \in W^{s, p}, \quad \varphi \in C^{\infty} .
$$

Then, $L$ is bounded and invertible.

Proof. By Lemma 34, the operator $L$ is semi-Fredholm, and moreover since $L$ is formally self-adjoint, it is Fredholm. It is well known that when the metric is smooth, the index of $L$ is zero independent of $s$ and $p$. We can approximate the metric $h$ by smooth metrics so that $L$ is arbitrarily close to a Fredholm operator with index zero. Since the level sets of index as a function on Fredholm operators are open, we conclude that the index of $L$ is zero. The injectivity of $L$ follows from Lemma 35(a), for if $\phi_{1}$ and $\phi_{2}$ are two solutions of $L \phi=g$, then the above lemma implies that $\phi_{1}-\phi_{2} \geqslant 0$ and $\phi_{2}-\phi_{1} \geqslant 0$. 
A.7. The Yamabe classification of nonsmooth metrics. Let $\mathcal{M}$ be a smooth, closed, connected $n$-dimensional Riemannian manifold with a smooth metric $h$, where we assume throughout this section that $n \geqslant 3$. With a positive scalar $\varphi$, let $\tilde{h}$ be related to $h$ by the conformal transformation $\tilde{h}=\varphi^{2^{\star}-2} h$, where $2^{\star}=\frac{2 n}{n-2}$. We say that $\tilde{h}$ and $h$ are conformally equivalent, and this defines an equivalence relation on the space of metrics. The equivalence class containing $h$ will be denoted by $[h]$; e.g., $h \in[h]$. It is well known that any smooth Riemannian metric $h$ on a given closed connected manifold $\mathcal{M}$ satisfies one and only one of the following three conditions:

$\mathcal{Y}^{+}:$There is a metric in $[h]$ with strictly positive scalar curvature;

$\mathcal{Y}^{0}$ : There is a metric in $[h]$ with vanishing scalar curvature;

$\mathcal{Y}^{-}$: There is a metric in $[h]$ with strictly negative scalar curvature.

These conditions define three disjoint classes in the space of metrics: they are referred to as the Yamabe classes.

We will extend the above classification to metrics in the Sobolev spaces $W^{s, p}$ under rather mild conditions on $s$ and $p$. Since the case $p=2$ is treated in [33] and the argument there easily extends to our slightly general setting, we shall only sketch the proof here. Given a Riemannian metric $h \in W^{s, p}$, let us consider the functional $E: W^{1,2} \rightarrow \mathbb{R}$ defined by

$$
E(\varphi)=(a \nabla \varphi, \nabla \varphi)+\left\langle R, \varphi^{2}\right\rangle
$$

where $a=4 \frac{n-1}{n-2}$. By Corollary 3, the pointwise multiplication is bounded on $W^{1,2} \otimes$ $W^{1,2} \rightarrow W^{\sigma, q}$ for $\sigma \leqslant 1$ and $\sigma-\frac{n}{q}<2-n$. Putting $\sigma=2-s$ and $q=p^{\prime}$, these conditions read as $2-s-\frac{n}{p^{\prime}}=2-n-s+\frac{n}{p}<2-n$ or $s-\frac{n}{p}>0$, and $s \geqslant 1$. So if $s p>n$ and $s \geqslant 1, \varphi^{2} \in W^{2-s, p^{\prime}}$ for $\varphi \in W^{1,2}$, meaning that the second term is bounded in $W^{1,2}$.

By using the functional $E$, we define the quantity

$$
\mu_{q}=\mu_{q}(h)=\inf _{\varphi \in B_{q}} E(\varphi), \quad \text { where } B_{q}=\left\{\varphi \in W^{1,2}:\|\varphi\|_{q}=1\right\} .
$$

Under the conditions $s p>n$ and $s \geqslant 1$, one can show that $\mu_{q}$ is finite for $q \geqslant 2$, and moreover that $\mu_{2^{\star}}$ is a conformal invariant, i.e., $\mu_{2^{\star}}(h)=\mu_{2^{\star}}(\tilde{h})$ for any two metrics $\tilde{h} \in[h]$, now allowing $W^{s, p}$ functions for the conformal factor. We refer to $\mu_{2^{\star}}(h)$ as the Yamabe invariant of the metric $h$, and we will see that the Yamabe classes correspond to the signs of the Yamabe invariant.

Theorem 11. Let $(\mathcal{M}, h)$ be a smooth, closed, connected Riemannian manifold with dimension $n \geqslant 3$ and with a metric $h \in W^{s, p}$, where we assume $s p>n$ and $s \geqslant 1$. Let $q \in\left[2,2^{\star}\right)$. Then, there exists $\phi \in W^{s, p}, \phi>0$ in $\mathcal{M}$, such that

$$
-a \Delta \phi+R \phi=\mu_{q} \phi^{q-1}, \quad \text { and } \quad\|\phi\|_{q}=1,
$$

where $\mu_{q}=\mu_{q}(h)$ is as defined above.

Proof. The above equation is the Euler-Lagrange equation for the functional $E$, so it suffices to show that $E$ attains its infimum $\mu_{q}$ over $B_{q}$ at a positive function $\phi \in W^{s, p}$. Let $\left\{\phi_{i}\right\} \subset B_{q}$ be a sequence satisfying $E\left(\phi_{i}\right) \rightarrow \mu_{q}$. From the continuity of the embed$\operatorname{ding} L^{q} \hookrightarrow L^{2}$, we have $\left\{\phi_{i}\right\}$ is bounded in $L^{2}$. It is the content of [33, Lemma 3.1] that

$$
E(\varphi) \geqslant C_{1}\|\varphi\|_{1,2}^{2}-C_{2}\|\varphi\|_{2}^{2}, \quad \varphi \in W^{1,2}
$$


for metrics in $W^{s, 2}$ with $s>\frac{n}{2}$. The proof works verbatim for our case, and since $\mu_{q}$ is finite, from this we conclude that $\left\{\phi_{i}\right\}$ is bounded in $W^{1,2}$. By the reflexivity of $W^{1,2}$ and the compactness of $W^{1,2} \hookrightarrow L^{q}$, there exist an element $\phi \in W^{1,2}$ and a subsequence $\left\{\phi_{i}^{\prime}\right\} \subset\left\{\phi_{i}\right\}$ such that $\phi_{i}^{\prime} \rightarrow \phi$ in $W^{1,2}$ and $\phi_{i}^{\prime} \rightarrow \phi$ in $L^{q}$. The latter implies $\phi \in B_{q}$. It is not difficult to show that $E$ is weakly lower semi-continuous, and it follows that $E(\phi)=\mu_{q}$, so $\phi$ satisfies (A.9). Bootstrapping with Corollary 5 implies that $\phi \in W^{s, p} \hookrightarrow W^{1, n}$, so that $|\phi| \in W^{1, n}$. Since $E(|\phi|)=E(\phi)$, after replacing $\phi$ by $|\phi|$, we can assume that $\phi \geqslant 0$. Finally, bootstrapping again gives $\phi \in W^{s, p}$, and since $\phi \neq 0$ as $\phi \in B_{q}$, by Lemma 35 we have $\phi>0$.

Under the conformal scaling $\tilde{h}=\varphi^{2^{\star}-2} h$, the scalar curvature transforms as

$$
\tilde{R}=\varphi^{1-2^{\star}}(-a \Delta \varphi+R \varphi),
$$

so assuming the conditions of the above theorem we infer that any given metric $h \in W^{s, p}$ can be transformed to the metric $\tilde{h}=\phi^{2^{\star}-2} h$ with the continuous scalar curvature $\tilde{R}=\mu_{q} \phi^{q-2^{\star}}$, where the conformal factor $\phi$ is as in the theorem. In other words, given any metric $h_{a b} \in W^{s, p}$, there exist continuous functions $\phi \in W^{s, p}$ with $\phi>0$ and $\tilde{R} \in W^{s, p}$ having constant sign, such that

$$
-a \Delta \phi+R \phi=\tilde{R} \phi^{2^{\star}-1} .
$$

We will prove below that the conformal class of the metric $h$ completely determines the sign of $\tilde{R}$, giving rise to the Yamabe classification of metrics in $W^{s, p}$.

In the class of smooth metrics there is a stronger result known as the Yamabe theorem: each conformal class of smooth metrics contains a metric with constant scalar curvature. The Yamabe theorem is a non-trivial extension of the above theorem to the critical case $q=2^{\star}$, and we see that for smooth metrics the sign of the Yamabe invariant determines which Yamabe class the metric is in. A proof of the Yamabe theorem requires more delicate techniques since we lose the compactness of the embedding $W^{1,2} \hookrightarrow L^{q}$, see e.g. [31] for a treatment of smooth metrics. As far as we know there has not appeared in the literature an explicit proof of the Yamabe theorem for nonsmooth metrics such as the ones considered in this paper, although it is generally expected to be true. We will not pursue this issue here; however, the following simpler result justifies the Yamabe classification of nonsmooth metrics.

Theorem 12. Let $(\mathcal{M}, h)$ be a smooth, closed, connected Riemannian manifold with dimension $n \geqslant 3$ and with a metric $h \in W^{s, p}$, where we assume $s p>n$ and $s \geqslant 1$. Then, the following hold:

- $\mu_{2^{\star}}>0$ iff there is a metric in $[h]$ with continuous positive scalar curvature.

- $\mu_{2^{\star}}=0$ iff there is a metric in [h] with vanishing scalar curvature.

- $\mu_{2 \star}<0$ iff there is a metric in $[h]$ with continuous negative scalar curvature.

In particular, two conformally equivalent metrics cannot have scalar curvatures with distinct signs.

Proof. We begin by proving that if there is a metric in $[h]$ with continuous scalar curvature of constant sign, then $\mu_{2^{\star}}$ has the corresponding sign. Since $\mu_{2 \star}$ is a conformal invariant, we can assume that the scalar curvature $R$ of $h$ is continuous and has constant sign. If $R<0$, then $E(\varphi)<0$ for constant test functions $\varphi=$ const and there 
is a constant function in $B_{2^{\star}}$, so we have $\mu_{2^{\star}}<0$. If $R \geqslant 0$, then $E(\varphi) \geqslant 0$ for any $\varphi \in W^{1,2}$, so $\mu_{2^{\star}} \geqslant 0$. Taking constant test functions, we infer that $R=0$ implies $\mu_{2^{\star}}=0$. Now, if $R>0$ then $E(\varphi)$ defines an equivalent norm on $W^{1,2}$, and we have $1=\|\varphi\|_{2^{\star}} \leqslant C\|\varphi\|_{1,2}$ for $\varphi \in B_{2^{\star}}$, so $\mu_{2^{\star}}>0$.

Next, we will prove that there is a metric in $[h]$ with continuous scalar curvature with the same sign as that of $\mu_{2^{\star}}$. To this end, for any $q \in\left[2,2^{\star}\right)$, we shall show that the sign of $\mu_{2^{\star}}$ determines the sign of $\mu_{q}$, so that the proof is completed by Theorem 11 . If $\mu_{2^{\star}}<0$, then $E(\varphi)<0$ for some $\varphi \in B_{2^{\star}}$, and since $E(k \varphi)=k^{2} E(\varphi)$ for $k \in \mathbb{R}$, there is some $k \varphi \in B_{q}$ such that $E(k \varphi)<0$, so $\mu_{q}<0$. If $\mu_{s^{\star}} \geqslant 0$, then $E(\varphi) \geqslant 0$ for all $\varphi \in B_{2^{\star}}$, and for any $\psi \in B_{q}$ there is $k$ such that $k \psi \in B_{2^{\star}}$, so $\mu_{q} \geqslant 0$. All such $k$ are uniformly bounded since $k=1 /\|\psi\|_{2^{\star}} \leqslant C /\|\psi\|_{q}=C$ by the continuity estimate $\|\cdot\|_{1} \leqslant C\|\cdot\|_{2^{\star}}$. From this we have for all $\psi \in B_{q}, E(\psi)=E(k \psi) / k^{2} \geqslant \mu_{2^{\star}} / k^{2} \geqslant \mu_{2^{\star}} / C^{2}$, meaning that $\mu_{2^{\star}}>0$ implies $\mu_{q}>0$. A similar scaling argument gives that if $\mu_{2^{\star}}=0$ then $\mu_{q}=0$.

A.8. Conformal covariance of the Hamiltonian constraint. Let $\mathcal{M}$ be a smooth, closed, connected $n$-dimensional manifold equipped with a Riemannian metric $h \in W^{s, p}$, where we assume throughout this section that $p \in(1, \infty), s \in\left(\frac{n}{p}, \infty\right) \cap[1, \infty)$ and that $n \geqslant 3$. We consider the Hamiltonian constraint

$$
H(\phi):=-\Delta \phi+\frac{1}{r(n-1)} R \phi+a_{\tau} \phi^{r+1}-a_{w} \phi^{-r-3}-a_{\rho} \phi^{-t}=0,
$$

where $r=\frac{4}{n-2}, t \in \mathbb{R}$ are constants, $R \in W^{s-2, p}$ is the scalar curvature of the metric $h$, and the other coefficients satisfy $a_{\tau}, a_{w}, a_{\rho} \in W_{+}^{s-2, p}$. In this Appendix, we will be interested in the transformation properties of $H$ under the conformal change $\tilde{h}=\theta^{r} h$ of the metric with the conformal factor $\theta \in W^{s, p}$ satisfying $\theta>0$. To this end, we consider

$$
\tilde{H}(\psi):=-\tilde{\Delta} \psi+\frac{1}{r(n-1)} \tilde{R} \psi+\tilde{a}_{\tau} \psi^{r+1}-\tilde{a}_{w} \psi^{-r-3}-\tilde{a}_{\rho} \psi^{-t}=0,
$$

where $\tilde{\Delta}$ is the Laplace-Beltrami operator associated to the metric $\tilde{h}, \tilde{R} \in W^{s-2, p}$ is the scalar curvature of $\tilde{h}$, and at the moment we do not impose any conditions on the remaining coefficients other than that they satisfy $\tilde{a}_{\tau}, \tilde{a}_{w}, \tilde{a}_{\rho} \in W_{+}^{s-2, p}$. One can derive the following relations:

$$
\begin{aligned}
\tilde{R} & =\theta^{-r} R-r(n-1) \theta^{-r-1} \Delta \theta, \\
\tilde{\Delta} \psi & =\theta^{-r} \Delta \psi+2 \theta^{-r-1} \nabla^{a} \theta \nabla_{a} \psi .
\end{aligned}
$$

Combining these relations with

$$
\Delta(\theta \psi)=\theta \Delta \psi+\psi \Delta \theta+2 \nabla^{a} \theta \nabla_{a} \psi,
$$

we obtain

$$
-\tilde{\Delta} \psi+\frac{1}{r(n-1)} \tilde{R} \psi=\theta^{-r-1}\left(-\Delta(\theta \psi)+\frac{1}{r(n-1)} R \theta \psi\right),
$$

which in turn implies that

$$
\tilde{H}(\psi)=\theta^{-r-1} H(\theta \psi),
$$


provided in the definition of $\tilde{H}$ that $\tilde{a}_{\tau}=a_{\tau}, \tilde{a}_{w}=\theta^{-2 r-4} a_{w}$, and $\tilde{a}_{\rho}=\theta^{-t-r-1} a_{\rho}$. We have proved the following well known result.

Lemma 37. Assume the above setting, so in particular, $\tilde{a}_{\tau}=a_{\tau}, \tilde{a}_{w}=\theta^{-2 r-4} a_{w}$, and $\tilde{a}_{\rho}=\theta^{-t-r-1} a_{\rho}$. Then we have

$$
\begin{aligned}
& \tilde{H}(\psi)=0 \quad \Leftrightarrow \quad H(\theta \psi)=0, \\
& \tilde{H}(\psi) \geqslant 0 \quad \Leftrightarrow \quad H(\theta \psi) \geqslant 0, \\
& \tilde{H}(\psi) \leqslant 0 \quad \Leftrightarrow \quad H(\theta \psi) \leqslant 0 .
\end{aligned}
$$

A.9. General conformal rescaling and the near-CMC condition. In this article we focused on the standard conformal method to produce the particular coupled elliptic PDE system that we analyzed. Here we examine briefly other decompositions to see if it is possible to remove the near-CMC obstacle for non-CMC existence that still seems to remain for the non-positive Yamabe classes and for the positive Yamabe class with large data.

The key question here is whether or not the standard conformal method essentially hard-wires the near-CMC assumption into the coupled system in order to get a domain of attraction for fixed-point iterations. If this is the case, then there remains the possibility that one can reverse-engineer a formulation, different from the conformal method, that gives a domain of attraction (preferably a contraction so that we also get uniqueness) without use of near-CMC conditions. Unfortunately, the answer appears to be negative, as we demonstrate below. In particular, it seems that the near-CMC obstacle is present in all possible formulations based on conformal transformations, if the estimate (5.1) is used.

To begin, recall that the objects $\left(\mathcal{M}, \hat{h}_{a b}, \hat{k}_{a b}, \hat{\rho}, \hat{j}_{a}\right)$ form an $n$-dimensional initial data set for Einstein's equations iff $\mathcal{M}$ is a $n$-dimensional smooth manifold, the tensor $\hat{h}_{a b}$ is a Riemannian metric on $\mathcal{M}$, the tensor $\hat{k}_{a b}$ is a symmetric tensor field on $\mathcal{M}$, the fields $\hat{\rho}$ and $\hat{j}_{a}$ are a non-negative scalar and a tensor field on $\mathcal{M}$, respectively, satisfying the condition $-\hat{\rho}^{2}+\hat{j}_{a} \hat{j}^{a}<0$, and the following equations hold:

$$
\begin{aligned}
\hat{R}+\hat{k}^{2}-\hat{k}_{a b} \hat{k}^{a b}-2 \kappa \hat{\rho} & =0, \\
-\hat{\nabla}_{a} \hat{k}^{a b}+\hat{\nabla}^{b} \hat{k}+\kappa \hat{j}^{b} & =0,
\end{aligned}
$$

where $\hat{\nabla}_{a}$ is the Levi-Civita connection of the metric $\hat{h}_{a b}$, the scalar field $\hat{R}$ is the Ricci scalar of the connection $\hat{\nabla}_{a}$, the scalar $\hat{k}=\hat{k}_{a b} \hat{h}^{a b}$ is the trace of the tensor $\hat{k}_{a b}$, and the constant $\kappa=8 \pi$ in units where both the gravitation constant $G$ and the speed of light $c$ have value one. The initial data set for Einstein's equations describe an instant of time in the physical world if we choose the number $n=3$. Nevertheless, in the calculations that follow we keep the number $n$ as a general positive integer.

Introduce the decomposition of the two-index tensor $k_{a b}$ into trace-free and trace parts, as follows:

$$
\hat{k}^{a b}=\hat{s}^{a b}+\frac{1}{n} \hat{k} \hat{h}^{a b}
$$

where $\hat{s}_{a b} \hat{h}^{a b}=0$. Introduce the following conformal rescaling:

$$
\hat{h}_{a b}=\phi^{r} h_{a b}, \quad \hat{s}^{a b}=\phi^{s} s^{a b}, \quad \hat{k}=\phi^{t} k,
$$


where the integers $r, s$, and $t$ are arbitrary, and we have introduced the Riemannian metric $h_{a b}$, a symmetric tensor $s^{a b}$, and a scalar field $k$. Introduce $\nabla_{a}$, the Levi-Civita connection of the metric $h_{a b}$, which satisfies the equation $\nabla_{a} h_{b c}=0$, and denote by $R$ the Ricci scalar of this connection $\nabla_{a}$. The rescaling above induces the following equations:

$$
\hat{h}^{a b}=\phi^{-r} h^{a b}, \quad \hat{s}_{a b}=\phi^{(2 r+s)} s_{a b},
$$

where $\hat{h}^{a b}$ is the inverse tensor of $\hat{h}_{a b}$, and $h^{a b}$ is the inverse tensor of $h_{a b}$. We use the convention that indices in all other hatted tensors are raised and lowered with the tensors $\hat{h}^{a b}$ and $\hat{h}_{a b}$, respectively, while indices on unhatted tensors are raised and lowered with the tensors $h^{a b}$ and $h_{a b}$, respectively. For example:

$$
\hat{s}_{a b}=\hat{h}_{a c} \hat{h}_{b d} \hat{s}^{c d}=\phi^{r} h_{a c} \phi^{r} h_{b d} \phi^{s} s^{c d}=\phi^{(2 r+s)} s_{a b} .
$$

The rescaling introduced in Eq. (A.13) implies that the tensor field $\hat{k}^{a b}$ transforms as follows:

$$
\hat{k}^{a b}=\phi^{s} s^{a b}+\frac{1}{n} \phi^{(t-r)} k h^{a b} \Leftrightarrow \hat{k}_{a b}=\phi^{(2 r+s)} s_{a b}+\frac{1}{n} \phi^{(t+r)} k h_{a b} .
$$

The connections $\hat{\nabla}_{a}$ and $\nabla_{a}$ differ in a tensor field $C_{a b}{ }^{c}$, in the sense that for any tensor field $v_{a}$,

$$
\hat{\nabla}_{a} v_{b}=\nabla_{a} v_{b}-C_{a b}{ }^{c} v_{c} \text { holds. }
$$

The tensor field $C_{a b}{ }^{c}$ depends on the scalar field $\phi$ and the number $r$ as follows:

$$
C_{a b}{ }^{c}=r \delta_{(a}^{c} \nabla_{b)} \ln (\phi)-\frac{r}{2} h_{a b} h^{c d} \nabla_{d} \ln (\phi) .
$$

This expression implies the contractions

$$
h^{a b} C_{a b}{ }^{c}=-\frac{r}{2}(n-2) h^{c d} \nabla_{d} \ln (\phi), \quad C_{a b}{ }^{b}=\frac{n r}{2} \nabla_{a} \ln (\phi) .
$$

Given any two connections $\hat{\nabla}_{a}$ and $\nabla_{a}$ related by a tensor field $C_{a b}{ }^{c}$, the Riemann, Ricci, and Ricci scalar fields associated with these two connections are related by the following expressions:

$$
\begin{aligned}
\hat{R}_{a b c}{ }^{d} & =R_{a b c}{ }^{d}-2 \nabla_{[a} C_{b] c}{ }^{d}+2 C_{c[a}{ }^{e} C_{b] e}{ }^{d}, \\
\hat{R}_{a c} & =R_{a c}-\nabla_{a} C_{c b}{ }^{b}+\nabla_{b} C_{a c}{ }^{b}+C_{c a}{ }^{e} C_{e b}{ }^{b}-C_{c b}{ }^{e} C_{a e}{ }^{b}, \\
\hat{R} & =\phi^{-r}\left[R-\nabla^{a} C_{a b}{ }^{b}+\nabla_{b}\left(h^{a c} C_{a c}{ }^{b}\right)+h^{a c} C_{c a}{ }^{e} C_{e b}{ }^{b}-h^{a c} C_{c b}{ }^{e} C_{a e}{ }^{b}\right],
\end{aligned}
$$

where indices between square brackets mean anti-symmetrization, that is, given any tensor $u_{a b}$ we define $u_{[a b]}:=\left(u_{a b}-u_{b a}\right) / 2$. In the case that the tensor $C_{a b}{ }^{c}$ is given by Eq. (A.14), the Ricci scalars $\hat{R}$ and $R$ satisfy the equation

$$
\hat{R}=\phi^{-(r+1)}\left[\phi R-r(n-1) \Delta \phi-\frac{r}{4 \phi}(n-1)[r(n-2)-4]\left(\nabla_{a} \phi\right)\left(\nabla^{a} \phi\right)\right] .
$$

Introduce the Hamiltonian and momentum fields,

$$
\begin{aligned}
\hat{H} & :=\hat{R}+\hat{k}^{2}-\hat{k}_{a b} \hat{k}^{a b}, \\
\hat{M}^{b} & :=-\hat{\nabla}_{a} \hat{k}^{a b}+\hat{\nabla}^{b} \hat{k},
\end{aligned}
$$


then the conformal rescaling given in Eq. (A.13) implies the following equations:

$$
\begin{aligned}
\hat{H}= & \phi^{-(r+1)}\left[\phi R-r(n-1) \Delta \phi-\frac{r}{4 \phi}(n-1)[r(n-2)-4]\left(\nabla_{a} \phi\right)\left(\nabla^{a} \phi\right)\right] \\
& +\frac{n-1}{n} \phi^{2 t} k^{2}-\phi^{2(r+s)} s_{a b} s^{a b}, \\
\hat{M}_{b}= & -\phi^{(r+s)} \nabla_{a} s_{b}{ }^{a}+\frac{n-1}{n} \phi^{t} \nabla_{b} k-\left(\frac{r n}{2}+r+s\right) \phi^{(r+s)} s_{b}{ }^{a} \nabla_{a} \ln (\phi) \\
& +\frac{n-1}{n} t \phi^{t} k \nabla_{b} \ln (\phi) .
\end{aligned}
$$

It is convenient to reorder the terms in these equations in such a way that the equation for the Hamiltonian field is given by

$$
\begin{aligned}
& -r(n-1) \Delta \phi-\frac{r}{4 \phi}(n-1)[r(n-2)-4]\left(\nabla_{a} \phi\right)\left(\nabla^{a} \phi\right) \\
& +R \phi+\frac{(n-1)}{n} k^{2} \phi^{(2 t+r+1)}-s_{a b} s^{a b} \phi^{(3 r+2 s+1)}=\phi^{(r+1)} \hat{H},
\end{aligned}
$$

and the equation for the momentum field is given by

$$
\begin{aligned}
& -\nabla_{a} s_{b}{ }^{a}-\left(\frac{(n+2)}{2} r+s\right) s_{b}{ }^{a} \nabla_{a} \ln (\phi) \\
& =\phi^{-(r+s)} \hat{M}_{b}-\frac{(n-1)}{n} \phi^{(t-r-s)} \nabla_{b} k-\frac{(n-1)}{n} t \phi^{(t-r-s-1)} k \nabla_{b} \phi .
\end{aligned}
$$

There are many interesting particular cases of the equations above. The first case is to keep the dimension $n \geqslant 3$ arbitrary, and choose:

$$
r=\frac{4}{n-2}, \quad s=-\frac{(n+2)}{2} r, \quad t=0,
$$

then, introducing the number $2^{*}:=2 n /(n-2)$, we conclude that the $n$-dimensional vacuum Einstein constraint equations $\left(H=0, M_{b}=0\right)$ can be written as follows:

$$
\begin{aligned}
& -\frac{4(n-1)}{(n-2)} \Delta \phi+R \phi+\frac{(n-1)}{n} k^{2} \phi^{\left(2^{*}-1\right)}-s_{a b} s^{a b} \phi^{-\left(2^{*}+1\right)}=0, \\
& -\nabla_{a} s_{b}{ }^{a}+\frac{(n-1)}{n} \phi^{2^{*}} \nabla_{b} k=0 .
\end{aligned}
$$

In the case that the manifold $\mathcal{M}$ is 3 -dimensional, we have the number $2^{*}=6$, and the equation for the Hamiltonian field is given by

$$
\begin{aligned}
& -2 r \Delta \phi-\frac{r}{2 \phi}(r-4)\left(\nabla_{a} \phi\right)\left(\nabla^{a} \phi\right) \\
& +R \phi+\frac{2}{3} k^{2} \phi^{(2 t+r+1)}-s_{a b} s^{a b} \phi^{(3 r+2 s+1)}=\phi^{(r+1)} \hat{H},
\end{aligned}
$$

and the equation for the momentum field is given by

$$
\begin{aligned}
& -\nabla_{a} s_{b}{ }^{a}-\left(\frac{3 r}{2}+r+s\right) s_{b}{ }^{a} \nabla_{a} \ln (\phi) \\
& =\phi^{-(r+s)} \hat{M}_{b}-\frac{2}{3} \phi^{(t-r-s)} \nabla_{b} k-\frac{2}{3} t \phi^{(t-r-s-1)} k \nabla_{b} \phi .
\end{aligned}
$$


The semi-decoupling decomposition in the case of the vacuum Einstein constraint equations $\left(H=0, M_{b}=0\right)$ is obtained from Eqs. (A.15)-(A.16) in the particular case of $r=4, s=-10$, and $t=0$, that is,

$$
\begin{aligned}
& -8 \Delta \phi+R \phi+\frac{2}{3} k^{2} \phi^{\left(2^{*}-1\right)}-s_{a b} s^{a b} \phi^{-\left(2^{*}+1\right)}=0, \\
& -\nabla_{a} s_{b}{ }^{a}+\frac{2}{3} \phi^{2^{*}} \nabla_{b} k=0 .
\end{aligned}
$$

The conformally covariant decomposition, in the case of the vacuum Einstein constraint equations $\left(H=0, M_{b}=0\right)$ and in the case that the transverse, traceless part of the tensor $k_{a b}$ vanishes, is obtained from Eqs. (A.15)-(A.16) with the particular choice of $r=4, s=-4$, and $t=0$, that is,

$$
\begin{aligned}
& -8 \Delta \phi+R \phi+\left(\frac{2}{3} k^{2}-s_{a b} s^{a b}\right) \phi^{\left(2^{*}-1\right)}=0, \\
& -\nabla_{a} s_{b}{ }^{a}-6 s_{b}{ }^{a} \nabla_{a} \ln (\phi)+\frac{2}{3} \nabla_{b} k=0 .
\end{aligned}
$$

As a final example, it is interesting to write down the rescaled equations above in the case $r=4, s=-10, t$ arbitrary:

$$
\begin{aligned}
& -8 \Delta \phi+R \phi+\frac{2}{3} \phi^{(2 t+5)} k^{2}-\phi^{-7} s_{a b} s^{a b}=\phi^{5} \hat{H}, \\
& -\nabla_{a} s_{b}{ }^{a}=\phi^{6} \hat{M}_{b}-\frac{2}{3} \phi^{(t+6)} \nabla_{b} k-\frac{2}{3} t \phi^{(t+5)} k \nabla_{b} \phi .
\end{aligned}
$$

Since the leading power in each equation scales exactly as the conformal method, the same argument leading to the negative result for the conformal method in Lemma 10 will apply here. Therefore, it appears that the different conformal rescalings produce coupled systems leading to precisely the same form of the near-CMC condition to establish non-CMC existence, in the case of both the non-positive Yamabe classes and the positive Yamabe class for large data.

Open Access This article is distributed under the terms of the Creative Commons Attribution Noncommercial License which permits any noncommercial use, distribution, and reproduction in any medium, provided the original author(s) and source are credited.

\section{References}

1. Allen, P., Clausen, A., Isenberg, J.: Near-constant mean curvature solutions of the Einstein constraint equations with non-negative Yamabe metrics. Class. Quant. Grav. 25, 075009 (2008)

2. Amann, H.: Fixed point equations and nonlinear eigenvalue problems in ordered Banach spaces. SIAM Review 18(4), 620-709 (1976)

3. Aubin, T.: Nonlinear Analysis on Manifolds. Monge-Ampère Equations. New York: Springer-Verlag, 1982

4. Bartnik, R., Fodor, G.: On the restricted validity of the thin sandwich conjecture. Phys. Rev. D 48(8), 3596-3599 (1993)

5. Bartnik, R., Isenberg J.: The constraint equations. In: Chruściel, P., Friedrich, H. eds., The Einstein equations and large scale behavior of gravitational fields. Berlin: Birhäuser, 2004, pp. 1-38

6. Beig, R.: TT-tensors and conformally flat structures on 3-manifolds. In: Chruściel, P.T. ed., Mathematics of Gravitation, Part 1, Volume 41. Warszawa: Banach Center Publications, Polish Academy of Sciences, Institute of Mathematics, 1997, pp. 109-118. Available at http://arXiv.org/abs/gr-qc/9606055v1, 1996 
7. Beig, R.: Generalized Bowen-York initial data. In: Cotsakis, S., Gibbons, G. eds., Mathematical and Quantum Aspects of Relativity and Cosmology, Volume 537. Springer Lecture Note in Physics, Berlin: Springer, 2000, pp. 55-69

8. Beig, R., Ó Murchadha, N.: The momentum constraints of general relativity and spatial conformal isometries. Commun. Math. Phys. 176(3), 723-738 (1996)

9. Bowen, J., York, J.: Time-asymmetric initial data for black holes and black-hole collisions. Phys. Rev. D 21(8), 2047-2055 (1980)

10. Choquet-Bruhat, Y.: Einstein constraints on compact $n$-dimensional manifolds. Class. Quant. Grav. 21, S127-S151 (2004)

11. Choquet-Bruhat, Y., Isenberg, J., York, J.: Einstein constraint on asymptotically Euclidean manifolds. Phys. Rev. D 61, 084034 (2000)

12. Corvino, J.: Scalar curvature deformation and a gluing construction for the Einstein constraint equations. Commun. Math. Phys. 214, 137-189 (2000)

13. Dain, S.: Initial data for a head on collision of two Kerr-like black holes with close limit. Phys. Rev. D 64(15), 124002 (2001)

14. Dain, S.: Initial data for two Kerr-like black holes. Phys. Rev. Lett. 87(12), 121102 (2001)

15. Dain, S.: Trapped surfaces as boundaries for the constraint equations. Class. Quant. Grav. 21(2), 555-573 (2004)

16. Du, Y.: Order structure and topological methods in nonlinear partial differential equations, Vol I. New Jersey, London, Singapore: World Scientific, 2006

17. Geroch, R., Traschen, J.: Strings and other distributional sources in general relativity. Phys. Rev. D 36(4), 1017-1031 (1987)

18. Grisvard, P.: Elliptic Problems in Nonsmooth Domains. Marshfield, MA: Pitman Publishing, 1985

19. Hebey, E.: Sobolev spaces on Riemannian manifolds, Volume $\mathbf{1 6 3 5}$ of Lecture notes in mathematics. Berlin, New York: Springer, 1996

20. Holst, M.: Adaptive numerical treatment of elliptic systems on manifolds. Adv. Comp. Math. 15, 139-191 (2001)

21. Holst, M., Nagy, G., Tsogtgerel, G.: Rough solutions of the Einstein constraints on manifolds with boundary. Preprint, available at http://arXiv.org/abs0712.0798v1[gr-qc], 2007

22. Holst, M., Nagy, G., Tsogtgerel, G.: Far-from-constant mean curvature solutions of Einstein's constraint equations with positive Yamabe metrics. Phys. Rev. Lett. 100(16), 161101.1-161101.4 (2008)

23. Holst, M., Tsogtgerel, G.: Adaptive finite element approximation of nonlinear geometric PDE. Preprint

24. Holst, M., Tsogtgerel, G.: Convergent adaptive finite element approximation of the Einstein constraints. Preprint

25. Isenberg, J.: Constant mean curvature solution of the Einstein constraint equations on closed manifold. Class. Quant. Grav. 12, 2249-2274 (1995)

26. Isenberg, J., Moncrief, V.: A set of nonconstant mean curvature solution of the Einstein constraint equations on closed manifolds. Class. Quant. Grav. 13, 1819-1847 (1996)

27. Isenberg, J., Ó Murchadha, N.: Non CMC conformal data sets which do not produce solutions of the Einstein constraint equations. Class. Quant. Grav. 21, S233-S242 (2004)

28. Isenberg, J., Park, J.: Asymptotically hyperbolic non-constant mean curvature solutions of the Einstein constraint equations. Class. Quant. Grav. 14, A189-A201 (1997)

29. Jerome, J.: Consistency of semiconductor modeling: an existence/stability analysis for the stationary van Roosbroeck system. SIAM J. Appl. Math. 45(4), 565-590 (1985)

30. Klainerman, S., Rodnianski, I.: Improved local well posedness for quasilinear wave equations in dimension three. Duke Math. J. 117(1), 1-124 (2003)

31. Lee, J., Parker, T.: The Yamabe problem. Bull. Amer. Math. Soc. 17(1), 37-91 (1987)

32. Lichnerowicz, A.: L'integration des équations de la gravitation relativiste et le problème des $\mathrm{n}$ corps. J. Math. Pures Appl. 23, 37-63 (1944)

33. Maxwell, D.: Rough solutions of the Einstein constraint equations on compact manifolds. J. Hyp. Diff. Eqs. 2(2), 521-546 (2005)

34. Maxwell, D.: Solutions of the Einstein constraint equations with apparent horizon boundaries. Commun. Math. Phys. 253(3), 561-583 (2005)

35. Maxwell, D.: Rough solutions of the Einstein constraint equations. J. Reine Angew. Math. 590, 1-29 (2006)

36. Maxwell, D.: A class of solutions of the vacuum einstein constraint equations with freely specified mean curvature. http://arXiv.org/abs/0804.0874v1[gr-qc], 2008

37. Misner, C., Thorne, K., Wheeler, J.: Gravitation. San Francisco, CA: W. H. Freeman and Company, 1970

38. Mitrović, D., Žubrinić, D.: Fundamentals of applied functional analysis, Volume 91 of Pitman monographs and surveys in pure and applied mathematics. Essex, UK: Addison Wesley Longman, 1998

39. Ó Murchadha, N., York, J.: Existence and uniqueness of solutions of the Hamiltonian constraint of general relativity on compact manifolds. J. Math. Phys. 14(11), 1551-1557 (1973) 
40. Ó Murchadha, N., York, J.: Initial-value problem of general relativity I. General formulation and physical interpretation. Phys. Rev. D 10(2), 428-436 (1974)

41. Ó Murchadha, N., York, J.: Initial-value problem of general relativity II. Stability of solution of the initial-value equations. Phys. Rev. D 10(2), 437-446 (1974)

42. Palais, R.: Seminar on the Atiyah-Singer index theorem. Princeton, NJ: Princeton University Press, 1965

43. Rosenberg, S.: The Laplacian on a Riemannian Manifold. Cambridge: Cambridge University Press, 1997

44. Rudin, W.: Real \& Complex Analysis. New York: McGraw-Hill, 1987

45. Schwarz, G.: Hodge decomposition - a method for solving boundary value problems. In: Lecture Notes in Mathematics, Volume 1607. Berlin-Heidelberg-New York: Springer Verlag, 1995

46. Triebel, H.: Theory of function spaces, Volume $\mathbf{7 8}$ of Monographs in Mathematics. Basel : Birkhäuser Verlag, 1983

47. Trudinger, N.: Linear elliptic operators with measurable coefficients. Ann. Scuola Norm. Sup. Pisa 27(3), 265-308 (1973)

48. Wald, R.: General Relativity. Chicago, IL: The University of Chicago Press, 1984

49. York, J.: Gravitational degrees of freedom and the initial-value problem. Phys. Rev. Lett. 26(26), $1656-1658$ (1971)

50. York, J.: Role of conformal three-geometry in the dynamics of gravitation. Phys. Rev. Lett. 28(16), 1082-1085 (1972)

51. York, J.: Conformally invariant orthogonal decomposition of symmetric tensor on Riemannian manifolds and the initial-value problem of general relativity. J. Math. Phys. 14(4), 456-464 (1973)

52. York, J.: Covariant decompositions of symmetric tensors in the theory of gravitation. Ann. Inst. Henri Poincare A 21(4), 319-332 (1974)

53. York, J.: Conformal "thin-sandwich" data for the initial-value problem of general relativity. Phys. Rev. Lett. 82, 1350-1353 (1999)

54. Zeidler, E.: Nonlinear Functional Analysis and its Applications I, Fixed-Point Theorems. New York: Springer, 1986

55. Zolesio, J.L.: Multiplication dans les espaces de Besov. Proc. Royal Soc. Edinburgh (A) 78(2), $113-117$ (1977)

Communicated by G. W. Gibbons 